\title{
YANGIANS, QUANTUM LOOP ALGEBRAS, AND ABELIAN DIFFERENCE EQUATIONS
}

\author{
SACHIN GAUTAM AND VALERIO TOLEDANO LAREDO
}

To Andrei Zelevinsky (1953-2013). Advisor, mentor, colleague, and friend.

\section{Contents}

1. Introduction

2. Yangians and quantum loop algebras

3. Integrable representations of $Y_{\hbar}(\mathfrak{g})$ and $U_{q}(L \mathfrak{g})$

4. Additive difference equations

5. The functor $\Gamma$

6. The inverse functor 7

7. q-Characters

Acknowledgments

References

Let $\mathfrak{g}$ be a complex, semisimple Lie algebra, and $Y_{\hbar}(\mathfrak{g})$ and $U_{q}(L \mathfrak{g})$ the Yangian and quantum loop algebra of $\mathfrak{g}$. Assuming that $\hbar$ is not a rational number and that $q=e^{\pi i \hbar}$, we construct an equivalence between the finite-dimensional representations of $U_{q}(L \mathfrak{g})$ and an explicit subcategory of those of $Y_{\hbar}(\mathfrak{g})$ defined by choosing a branch of the logarithm. This equivalence is governed by the monodromy of the abelian, additive difference equations defined by the commuting fields of $Y_{\hbar}(\mathfrak{g})$. Our results are compatible with $q$-characters, and apply more generally to a symmetrizable Kac-Moody algebra $\mathfrak{g}$, in particular to affine Yangians and quantum toroïdal algebras. In this generality, they yield an equivalence between the representations of $Y_{\hbar}(\mathfrak{g})$ and $U_{q}(L \mathfrak{g})$ whose restriction to $\mathfrak{g}$ and $U_{q} \mathfrak{g}$, respectively, are integrable and in category $\mathcal{O}$.

\section{INTRODUCTION}

1.1. Let $\mathfrak{g}$ be a complex semisimple Lie algebra, and $Y_{\hbar}(\mathfrak{g})$ and $U_{q}(L \mathfrak{g})$ the Yangian and quantum loop algebra of $\mathfrak{g}$. Recall that the latter are deformations of the enveloping algebras of the current Lie algebra $\mathfrak{g}[s]$ of $\mathfrak{g}$ and its loop algebra $\mathfrak{g}\left[z, z^{-1}\right]$, respectively. We shall assume throughout that $q$ is not a root of unity, and that $e^{\pi \iota \hbar}=q$.

In this paper, we solve the long-standing problem of relating the finite-dimensional representations of $Y_{\hbar}(\mathfrak{g})$ and $U_{q}(L \mathfrak{g})$, thus giving a precise formulation to the

Received by the editors May 14, 2015.

2010 Mathematics Subject Classification. Primary 17B37; Secondary 17B67, 39A10, 82B43.

The second author was supported in part by NSF Grants DMS-0854792, DMS-1206305, and PHY-1066293. 
widely held belief that these quantum groups have the same representation theory (see, e.g., [51, p. 283]). Our solution is entirely explicit and is expressed in terms of

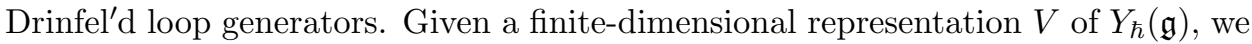
construct an action of $U_{q}(L \mathfrak{g})$ on $V$ by contour integral formulas, and vice versa. In particular, this gives rise to an exact, faithful functor

$$
\Gamma: \operatorname{Rep}_{\mathrm{fd}}\left(Y_{\hbar}(\mathfrak{g})\right) \longrightarrow \operatorname{Rep}_{\mathrm{fd}}\left(U_{q}(L \mathfrak{g})\right),
$$

which commutes with the forgetful functor to vector spaces. The functor $\Gamma$ may be thought of as an exponential cover in that it maps simple objects to simple objects, exponentiates the roots of their Drinfel'd polynomials, and restricts to an equivalence on the subcategory of $\operatorname{Rep}_{\mathrm{fd}}\left(Y_{\hbar}(\mathfrak{g})\right)$ obtained by requiring these roots to lie in a domain $\Pi \subset \mathbb{C}$ mapping bijectively to $\mathbb{C}^{\times}$via the map $u \rightarrow e^{2 \pi \iota u}$.

1.2. Our results apply more generally to an arbitrary symmetrizable Kac-Moody algebra $\mathfrak{g}$, in particular to affine Yangians and quantum toroïdal algebras, and are proved in that generality in the main body of the paper. When $\mathfrak{g}$ is infinitedimensional, the relevant categories consist of representations of $Y_{\hbar}(\mathfrak{g}), U_{q}(L \mathfrak{g})$ whose restrictions to $\mathfrak{g}$ and $U_{q} \mathfrak{g}$, respectively, are integrable and in category $\mathcal{O}$. For simplicity of exposition, however, we assume that $\mathfrak{g}$ is finite-dimensional for the rest of this Introduction.

1.3. Yangians and quantum loop algebras have been known to be closely related since their introduction by Drinfel'd in the mid 1980s [14,15. In particular,

(1) Drinfel'd proved that, just as the loop algebra $\mathfrak{g}\left[z, z^{-1}\right]$ degenerates to the current algebra $\mathfrak{g}[s]$ by setting $z=\exp (\epsilon s)$ and letting $\epsilon \rightarrow 0$, the quantum loop algebra degenerates to the Yangian. Specifically, $Y_{\hbar}(\mathfrak{g})$ is the graded algebra associated to the filtration of $U_{q}(L \mathfrak{g})$ by powers of the ideal of $z=1$ (see [15, and also [30] for a complete proof).

(2) The highest weight classification of simple modules due to Drinfel'd and Chari-Pressley [10, 16] yields a surjection from the parameters of finitedimensional irreducible representations of $Y_{\hbar}(\mathfrak{g})$ to those of $U_{q}(L \mathfrak{g})$. This (set-theoretic) map $\mathcal{E x p}$ is given by exponentiating the roots of Drinfel'd polynomials.

(3) Stronger results can be obtained when $\mathfrak{g}$ is of type ADE, by using Nakajima quiver varieties, and the geometric action of $U_{q}(L \mathfrak{g})$ on their equivariant $K$-theory [44]. The analogous action of the Yangian $Y_{\hbar}(\mathfrak{g})$ in cohomology due to Varagnolo [51] allows one to relate irreducible representations of $U_{q}(L \mathfrak{g})$ and $Y_{\hbar}(\mathfrak{g})$ via the Chern character, and shows in particular that the map $\mathcal{E} x p$ preserves dimensions.

1.4. While (1) does not a priori suggest the existence of a link between the representations of $U_{q}(L \mathfrak{g})$ and $Y_{\hbar}(\mathfrak{g})$ (there is after all no relation between the representations of a filtered algebra and of its associated graded in general), (2) and (3) clearly do, as does the fact that, in type A, evaluation representations of $Y_{\hbar}(\mathfrak{g})$ can be explicitly deformed to representations of $U_{q}(L \mathfrak{g})$. Such a link is also implicit in the conjecture of Frenkel-Reshetikhin according to which the monodromy of the difference analog of the Knizhnik-Zamolodchikov equations (qKZ) defined by the $R$-matrix of $Y_{\hbar}(\mathfrak{g})$ is described by the $R$-matrix of $U_{q}(L \mathfrak{g})$ 25]. 
The belief that finite-dimensional representations of $Y_{\hbar}(\mathfrak{g})$ and $U_{q}(L \mathfrak{g})$ should be related is further corroborated by the fact that, in the analogous case of the degenerate and affine Hecke algebras $\mathfrak{H}, \mathbb{H}$ of a Weyl group, a complete understanding of the relation between finite-dimensional representations of $\mathfrak{H}$ and $\mathbb{H}$ was obtained by Lusztig [40], and can be recovered by realizing $\mathfrak{H}, \mathbb{H}$ as the equivariant cohomology and $K$-theory of the corresponding Steinberg variety [29].

1.5. Despite the above evidence, however, no precise, functorial relation between the categories $\operatorname{Rep}_{\mathrm{fd}}\left(Y_{\hbar}(\mathfrak{g})\right)$ and $\operatorname{Rep}_{\mathrm{fd}}\left(U_{q}(L \mathfrak{g})\right)$ was known so far, even conjecturally 1 One notable exception is the works [18, 19, 35] which relate $U_{q}\left(L \mathfrak{s l}_{n}\right)$ and Felder's elliptic quantum group $E_{\tau, \gamma}\left(\mathfrak{g l}_{n}\right)$ and construct in particular a fully faithful functor $\mathcal{F}$ from $\operatorname{Rep}_{\mathrm{fd}}\left(U_{q}\left(L \mathfrak{s l}_{n}\right)\right)$ to $\operatorname{Rep}_{\mathrm{fd}}\left(E_{\tau, \gamma}\left(\mathfrak{g l}_{n}\right)\right)$ [19. A similar construction should give rise to a functor $F: \operatorname{Rep}_{\mathrm{fd}}\left(Y_{\hbar}\left(\mathfrak{s l}_{n}\right)\right) \rightarrow \operatorname{Rep}_{\mathrm{fd}}\left(U_{q}\left(L \mathfrak{g l}_{n}\right)\right)$. These constructions depend, however, on the RTT presentation of $U_{q}\left(L \mathfrak{s l}_{n}\right)$, which is less convenient for other classical Lie algebras and is currently unavailable for exceptional ones. Moreover, even in type A, the essential surjectivity of the functors $\mathcal{F}$ and $F$ seems difficult to establish and may in fact fail for numerical values of $\tau, q$.

1.6. Our results are in a sense optimal, short of proving that $U_{q}(L \mathfrak{g})$ and $Y_{\hbar}(\mathfrak{g})$ are isomorphic. An elementary analogy may be seen by looking at the exponential covering $\mathbb{C} \longrightarrow \mathbb{C}^{\times}$. The latter is a local isomorphism and possesses a global section for any determination of the logarithm.

The analogous local statement for $Y_{\hbar}(\mathfrak{g})$ and $U_{q}(L \mathfrak{g})$ was obtained in our previous work. Indeed, we proved in [26] that, when the deformation parameters $\hbar, q$ are formal, the quantum loop algebra and Yangian are isomorphic as algebras after appropriate completions, specifically with respect to the natural loop grading on $Y_{\hbar}(\mathfrak{g})$ and the powers of the ideal of $z=1$ in $U_{q}(L \mathfrak{g})$. The isomorphism

$$
\Phi: \widehat{U_{q}(L \mathfrak{g})} \longrightarrow \widehat{Y_{\hbar}(\mathfrak{g})}
$$

constructed in [26] deforms the change of variables $\mathfrak{g}\left[z, z^{-1}\right] \rightarrow \mathfrak{g}[[s]]$ given by $z=$ $e^{2 \pi \iota s}$ and exponentiates the roots of Drinfel'd polynomials.

The framework of [26] is, however, not suited for the study of finite-dimensional representations since the isomorphism $\Phi$ is given by formal power series which do not converge on these representations and do not allow for the numerical specialization of the deformation parameters.

1.7. Our first main result is the following theorem.

Theorem. If $\hbar \in \mathbb{C} \backslash \mathbb{Q}$ and $q=e^{\pi i \hbar}$, there is an exact, faithful functor

$$
\Gamma: \operatorname{Rep}_{\mathrm{fd}}\left(Y_{\hbar}(\mathfrak{g})\right) \longrightarrow \operatorname{Rep}_{\mathrm{fd}}\left(U_{q}(L \mathfrak{g})\right),
$$

which commutes with the forgetful functor to vector spaces, maps simple objects to simple objects, and exponentiates the roots of Drinfel'd polynomials 2

\footnotetext{
${ }^{1}$ The Chern character in the geometric realizations (3) above cannot be considered a functor, since it is not known how to realize arbitrary finite-dimensional representations of $Y_{\hbar}(\mathfrak{g})$ of $U_{q}(L \mathfrak{g})$ inside the equivariant cohomology or $K$-theory of a quiver variety.

${ }^{2}$ Strictly speaking, the functor $\Gamma$ is only defined on the dense subcategory of non-congruent representations of $Y_{\hbar}(\mathfrak{g})$ introduced in Section 5 See Section 5.4 for a precise statement of Theorem 1.7. We shall gloss over this point in the introduction.
} 
1.8. Our second main result asserts the existence of an inverse to $\Gamma$ for each determination of the logarithm. Specifically, fix a subset $\Pi \subset \mathbb{C}$ such that

$$
(\Pi-\Pi) \cap \mathbb{Z}=\{0\} \quad \text { and } \quad \Pi \pm \frac{\hbar}{2} \subset \Pi .
$$

The map $u \mapsto e^{2 \pi \iota u}$ is then a bijection between $\Pi$ and a subset $\Omega \subset \mathbb{C}^{\times}$stable under multiplication by $q^{ \pm 1}$. To such a choice, we attach categories

$$
\operatorname{Rep}_{\mathrm{fd}}^{\Pi}\left(Y_{\hbar}(\mathfrak{g})\right) \text { and } \operatorname{Rep}_{\mathrm{fd}}^{\Omega}\left(U_{q}(L \mathfrak{g})\right)
$$

consisting of representations such that the roots of their Drinfel'd polynomials lie in $\Pi$ and $\Omega$, respectively.

\section{Theorem.}

(1) The functor $\Gamma$ restricts to a functor

$$
\Gamma_{\Pi}: \operatorname{Rep}_{\mathrm{fd}}^{\Pi}\left(Y_{\hbar}(\mathfrak{g})\right) \longrightarrow \operatorname{Rep}_{\mathrm{fd}}^{\Omega}\left(U_{q}(L \mathfrak{g})\right) .
$$

(2) The functor $\Gamma_{\Pi}$ admits an inverse functor

$$
\urcorner_{\Pi}: \operatorname{Rep}_{\mathrm{fd}}^{\Omega}\left(U_{q}(L \mathfrak{g})\right) \longrightarrow \operatorname{Rep}_{\mathrm{fd}}^{\Pi}\left(Y_{\hbar}(\mathfrak{g})\right) .
$$

In particular, if $\Pi$ is a fundamental domain for $u \rightarrow u+1$, $\Gamma$ restricts to an equivalence between $\operatorname{Rep}_{\mathrm{fd}}^{\Pi}\left(Y_{\hbar}(\mathfrak{g})\right)$ and $\operatorname{Rep}_{\mathrm{fd}}\left(U_{q}(L \mathfrak{g})\right)$.

1.9. The novel idea underlying our construction is that each of the commuting fields of $Y_{\hbar}(\mathfrak{g})$ defines a difference equation on any finite-dimensional representation of $Y_{\hbar}(\mathfrak{g})$. The functor $\Gamma$ is governed by the monodromy of this equation, an averaging which yields a canonical means of replacing the rational matrix coefficients of $Y_{\hbar}(\mathfrak{g})$ by their trigonometric counterparts for $U_{q}(L \mathfrak{g})$ (for the reader's convenience, a selfcontained account of Birkhoff's theory of difference equations is given in Section 4). The abelian nature of these equations is essential in two respects. On the one hand, it leads to explicit formulas for the action of $U_{q}(L \mathfrak{g})$ on $Y_{\hbar}(\mathfrak{g})$-modules in terms of $\Gamma$-functions. On the other, it guarantees that the corresponding Riemann-Hilbert problem is always solvable, and therefore that $\Gamma$ is essentially surjective.

1.10. To illustrate this construction, it is useful to compare the simplest matrix coefficients of $Y_{\hbar}(\mathfrak{g})$ and $U_{q}(L \mathfrak{g})$ when $\mathfrak{g}=\mathfrak{s l}_{2}$. Let $V(a), \mathcal{V}(\mathbf{a})$ be the representations of $Y_{\hbar}\left(\mathfrak{s l}_{2}\right), U_{q}\left(L \mathfrak{s l}_{2}\right)$ obtained by evaluating the vector representations of $\mathfrak{s l}_{2}$ and $U_{q} \mathfrak{s l}_{2}$ at $a \in \mathbb{C}$ and $\mathbf{a} \in \mathbb{C}^{\times}$, respectively. If

$$
\xi(u)=1+\hbar \sum_{r \geq 0} \xi_{r} u^{-u-1} \quad \text { and } \quad \Psi(z)^{ \pm}=\sum_{k \geq 0} \Psi_{ \pm k}^{ \pm} z^{\mp k}
$$

are the generating functions of the commuting loop generators of $Y_{\hbar}\left(\mathfrak{s l}_{2}\right)$ and $U_{q}\left(L \mathfrak{s l}_{2}\right)$, respectively, the action of $\xi(u), \Psi(z)^{ \pm}$on the highest weight vectors $\omega \in V(a), \Omega \in \mathcal{V}(\mathbf{a})$ is given, respectively, by

$$
\xi(u) \omega=\frac{u+\hbar-a}{u-a} \omega \text { and } \Psi(z)^{ \pm} \Omega=q^{-1} \frac{q^{2} z-\mathbf{a}}{z-\mathbf{a}} \Omega .
$$

These formulas are tantalizingly close, yet only seem to be related by the mechanical, termwise exponentiation $z=e^{2 \pi \iota u}, \mathbf{a}=e^{2 \pi \iota a}$. Our construction in this case amounts to the observation that the matrix coefficient $\psi(z)=\left(q z-q^{-1} \mathbf{a}\right) /(z-\mathbf{a})$ is the monodromy of the additive difference equation $f(u+1)=A(u) f(u)$ determined 
by the matrix $A(u)=(u+\hbar-a) /(u-a)$. In other words, $\psi(z)$ is given by the averaging

$$
\frac{q z-q^{-1} \mathbf{a}}{z-\mathbf{a}}=\cdots \frac{u+1+\hbar-a}{u+1-a} \cdot \frac{u+\hbar-a}{u-a} \cdot \frac{u-1+\hbar-a}{u-1-a} \cdots .
$$

1.11. This observation may in fact be used to define an action of the commuting generators of $U_{q}(L \mathfrak{g})$, not only on the highest weight vector of a simple module but also on an arbitrary finite-dimensional representation $V$ of $Y_{\hbar}(\mathfrak{g})$ for any semisimple $\mathfrak{g}$, and in a way which is consistent with taking $q$-characters.

Specifically, for any vertex $i$ of the Dynkin diagram $\mathbf{I}$ of $\mathfrak{g}$, the generating function

$$
\xi_{i}(u)=1+\hbar \sum_{r \geq 0} \xi_{i, r} u^{-u-1} \in G L(V)\left[\left[u^{-1}\right]\right]
$$

of the corresponding commuting elements of $Y_{\hbar}(\mathfrak{g})$ can be shown to be the expansion at $u=\infty$ of an $\operatorname{End}(V)$-valued rational function. One can therefore consider the additive difference equation

$$
\phi_{i}(u+1)=\xi_{i}(u) \phi_{i}(u)
$$

determined by each $\xi_{i}(u)$, where the unknown $\phi_{i}$ is a meromorphic function of $u \in \mathbb{C}$ with values in $\operatorname{End}(V)$. This equation admits two fundamental solutions $\phi_{i}^{ \pm}(u)$ which are uniquely determined by the following requirements:

- $\phi_{i}^{ \pm}(u)$ is holomorphic and invertible for $\pm \operatorname{Re}(u) \gg 0$,

- $\phi_{i}^{ \pm}(u)$ is asymptotic to $\left(1+O\left(u^{-1}\right)\right)( \pm u)^{\hbar \xi_{i, 0}}$ as $u \rightarrow \infty$ with $\pm \operatorname{Re}(u) \gg 0$.

The solutions $\phi_{i}^{+}(u), \phi_{i}^{-}(u)$ are regularizations of the formal infinite products

$$
\xi_{i}(u)^{-1} \xi_{i}(u+1)^{-1} \cdots \quad \text { and } \xi_{i}(u-1) \xi_{i}(u-2) \cdots,
$$

which symbolically solve (1.1).

Following Birkhoff [6], one considers the monodromy matrix of the equation, that is, the $G L(V)$-valued function defined by

$$
S_{i}(u)=\phi_{i}^{+}(u)^{-1} \cdot \phi_{i}^{-}(u) .
$$

$S_{i}(u)$ is a regularization of the infinite product

$$
\cdots \xi_{i}(u+1) \xi_{i}(u) \xi_{i}(u-1) \cdots
$$

and, by construction, is a 1-periodic function of $u$. The asymptotics of $\phi_{i}^{ \pm}$further imply that $S_{i}$ is a rational function of the variable $z=e^{2 \pi \iota u}$, regular at $z=\infty, 0$, and such that $S_{i}(\infty)=e^{\pi \iota \hbar \xi_{i, 0}}=S_{i}(0)^{-1}$.

The action of the generating series

$$
\Psi_{i}(z)^{ \pm}=\sum_{k \geq 0} \Psi_{i, \pm k}^{ \pm} z^{\mp k}
$$

of the commuting generators of $U_{q}(L \mathfrak{g})$ on the $Y_{\hbar}(\mathfrak{g})$-module $V$ is then given by the Taylor series of the monodromy matrix $S_{i}(z)$ at $z=\infty, 0$. In the example described in 1.10, the (matrix elements of the) Birkhoff solutions are given by

$$
\phi^{+}(u)=\frac{\Gamma(u+\hbar-a)}{\Gamma(u-a)} \quad \text { and } \quad \phi^{-}(u)=\frac{\Gamma(1-u+a)}{\Gamma(1-u-\hbar+a)},
$$


where $\Gamma$ is Euler's Gamma function. The corresponding monodromy is

$$
S(z)=\frac{\Gamma(u-a)}{\Gamma(u+\hbar-a)} \cdot \frac{\Gamma(1-u+a)}{\Gamma(1-u-\hbar+a)}=\frac{q z-q^{-1} \mathbf{a}}{z-\mathbf{a}},
$$

where we used the identity $\Gamma(u) \Gamma(1-u)=\pi / \sin (\pi u)$ [52, Section 12.14].

1.12. The difference equation (1.1) can also be used to define an action of the raising and lowering loop generators $\left\{\mathcal{X}_{i, k}^{ \pm}\right\}_{i \in \mathbf{I}, k \in \mathbb{Z}}$ of $U_{q}(L \mathfrak{g})$ on the finite-dimensional $Y_{\hbar}(\mathfrak{g})$-module $V$ as follows. For each $i \in \mathbf{I}$ and $k \in \mathbb{Z}, \mathcal{X}_{i, k}^{ \pm}$acts as the operator defined by the contour integral

$$
\frac{c_{i}}{2 \pi \iota} \int_{\mathcal{C}_{i}^{ \pm}} e^{2 \pi \iota k u} g_{i}^{ \pm}(u) x_{i}^{ \pm}(u) d u,
$$

where

- $x_{i}^{ \pm}(u)=\hbar \sum_{r \geq 0} x_{i, r}^{ \pm} u^{-r-1}$ is the generating function of the raising/lowering generators of $Y_{\hbar}(\mathfrak{g})$, and acts on $V$ as a rational function of $u$.

- The functions $g_{i}^{+}(u)$ and $g_{i}^{-}(u)$ are regularizations of the products

$$
\cdots \xi_{i}(u+2) \xi_{i}(u+1) \text { and } \xi_{i}(u-1) \xi_{i}(u-2) \cdots,
$$

respectively, and are given in terms of the fundamental solutions $\phi_{i}^{ \pm}(u)$ of the difference equation (1.1) by

$$
g_{i}^{+}(u)=\phi_{i}^{+}(u+1)^{-1} \text { and } g_{i}^{-}(u)=\phi_{i}^{-}(u) .
$$

- The constant $c_{i}$ is given in terms of the symmetrizing integers $d_{i}$ of the Cartan matrix of $\mathfrak{g}$ by $c_{i}=\sqrt{d_{i}} \Gamma\left(d_{i} \hbar\right)$.

- The contour $\mathcal{C}_{i}^{ \pm}$contains all the poles of $x_{i}^{ \pm}(u)$, and none of their $\mathbb{Z}_{\neq 0^{-}}$ translates.

The proof that the above operators satisfy the commutation relations of $U_{q}(L \mathfrak{g})$ follows from contour integral manipulations reminiscent of those used in conformal field theory. One notable exception, however, is that the integrands have singularities on the shifted diagonals $u-v=a$, where $a$ is an integral multiple of $\hbar / 2$.

1.13. The construction of the inverse functor

$$
\urcorner_{\Pi}: \operatorname{Rep}_{\mathrm{fd}}^{\Omega}\left(U_{q}(L \mathfrak{g})\right) \longrightarrow \operatorname{Rep}_{\mathrm{fd}}^{\Pi}\left(Y_{\hbar}(\mathfrak{g})\right)
$$

given by Theorem 1.8 is obtained in a similar spirit by solving the inverse monodromy problem corresponding to the difference equation (1.1). It is worth stressing that, as for its differential counterpart, this Riemann-Hilbert problem possesses a solution for any monodromy matrix only because Equations (1.1) are abelian. This property is therefore crucial in establishing the essential surjectivity of the functor $\Gamma$.

Specifically, let $\mathcal{V} \in \operatorname{Rep}_{\mathrm{fd}}\left(U_{q}(L \mathfrak{g})\right)$, and consider the $\operatorname{End}(\mathcal{V})$-valued generating series $\Psi_{i}(z)^{ \pm}$given by (1.2),

$$
\mathcal{X}_{i}^{ \pm}(z)^{+}=\sum_{k \geq 0} \mathcal{X}_{i, k}^{ \pm} z^{-k} \quad \text { and } \quad \mathcal{X}_{i}^{ \pm}(z)^{-}=-\sum_{k \geq 1} \mathcal{X}_{i,-k}^{ \pm} z^{k} .
$$

It is well-known that these are the expansions at $z=\infty, 0$ of rational functions $\Psi_{i}(z)$ and $\mathcal{X}_{i}^{ \pm}(z)$ [5, 32. We show in Section 3 that $\mathcal{V}$ is in $\operatorname{Rep}_{\mathrm{fd}}^{\Omega}\left(U_{q}(L \mathfrak{g})\right)$ if and only if the poles of $\Psi_{i}(z)^{ \pm 1}$ and $\mathcal{X}_{i}^{ \pm}(z)$ are contained in $\Omega$. 
The operators giving the action of $Y_{\hbar}(\mathfrak{g})$ on $\rceil_{\Pi}(\mathcal{V})=\mathcal{V}$ are then defined as follows:

(1) As explained in Section 4 there is a unique rational function $\mathbb{C} \rightarrow G L(V)$, which we take to be $\xi_{i}(u)$, such that

- $\xi_{i}(u)=1+\hbar d_{i} \alpha_{i}^{\vee} u^{-1}+\cdots$;

- the connection matrix of the difference equation (1.1) defined by $\xi_{i}(u)$ is $S_{i}(z)=\Psi_{i}(z)$;

- the poles of $\xi_{i}(u)^{ \pm 1}$ lie in $\Pi$.

(2) Let $\phi_{i}^{ \pm}(u)$ be the canonical fundamental solutions of (1.1), and define the functions $g_{i}^{ \pm}(u)$ by (1.3). Then, for any $i \in \mathbf{I}$ and $r \in \mathbb{N}$, the generators $x_{i, r}^{ \pm}$act on $\mathcal{V}$ as the operators

$$
\frac{1}{c_{i} \hbar} \int_{\mathcal{C}_{i}^{ \pm}} v^{-r} g_{i}^{ \pm}(v)^{-1} \mathcal{X}_{i}^{ \pm}\left(e^{2 \pi \iota v}\right) d v
$$

where the constants $c_{i}$ are as in Section 1.12, and the contours $\mathcal{C}_{i}^{ \pm}$enclose all the poles of $\mathcal{X}_{i}^{ \pm}\left(e^{2 \pi \iota v}\right)$ contained in $\Pi$ and none of their $\mathbb{Z}_{\neq 0}$-translates.

1.14. The relevance of difference equations to Yangians and quantum loop algebras is widely known. For example, the $R$-matrix of $Y_{\hbar}(\mathfrak{g})$ (resp. $U_{q}(L \mathfrak{g})$ ) gives rise to the additive (resp. multiplicative) $q \mathrm{KZ}$ equations satisfied by products of intertwiners [25, 47]. In a related vein, Baxter's $Q$-operator for $Y_{\hbar}(\mathfrak{g})\left(\operatorname{resp} . U_{q}(L \mathfrak{g})\right)$ satisfies the TQ relations, which are difference equations with the step a multiple of $\hbar$ (resp. a power of $q$ ) (see, e.g., [1, 4, 23]). Moreover, the idea of averaging a rational or trigonometric solution of the quantum Yang-Baxter equations to obtain a trigonometric or elliptic solution has also been extensively used [2, 3, 20, 48,

It is worth pointing out that the difference equations used in this paper are not of $q \mathrm{KZ}$ or TQ type. Indeed, they are abelian and therefore far simpler. Their relevance seem to be new, although they are very closely related to the $\Gamma$-factors which appear in quantum cohomology [34,45].

1.15. The use of abelian difference equations to define a functor between finitedimensional representations of $Y_{\hbar}(\mathfrak{g})$ and $U_{q}(L \mathfrak{g})$ is the main discovery of the present paper. This is a potentially far reaching principle, which we have so far applied to two further problems:

(1) The construction of a tensor structure on the functor $\Gamma$, which yields a Kazhdan-Lusztig equivalence of meromorphic braided tensor categories between $Y_{\hbar}(\mathfrak{g})$ and $U_{q}(L \mathfrak{g})$, when both are endowed with the deformed Drinfel'd coproduct and the commutative part $R_{0}$ of the $R$-matrix. This tensor structure arises from the monodromy of the abelian $q \mathrm{KZ}$ equations defined by $R_{0}$ of $Y_{\hbar}(\mathfrak{g})$ [27.

(2) The construction of a faithful functor $\Theta$ between finite-dimensional representations of $U_{q}(L \mathfrak{g})$ and of Felder's elliptic quantum group [28. The functor $\Theta$ is governed by the monodromy of the difference equations defined by the commuting fields of $U_{q}(L \mathfrak{g})$. This is a very promising avenue, since elliptic quantum groups and their representations are not very well understood outside of type A 22 . 
We also plan to apply similar ideas to relate the affine Yangian $Y_{\hbar}\left(\widehat{\mathfrak{g l}_{1}}\right)$ appearing in the recent work of Maulik-Okounkov 42 to the quantum toroïdal algebra $U_{q}\left(\widehat{\mathfrak{a l}_{1}}\right)$ [21,43,46] (see also [49], where an isomorphism between appropriate completions of $U_{q}\left(\widehat{\mathfrak{g l}_{1}}\right)$ and $Y_{\hbar}\left(\widehat{\mathfrak{g l}}_{1}\right)$ is obtained in the formal setting by using the method of [26]).

1.16. The picture described in this paper is consistent with Cherednik's philosophy of obtaining equivalences of appropriate categories of finite-dimensional representations of the affine Hecke algebra $\mathbb{H}$ and degenerate affine Hecke algebra $\mathfrak{H}$ of a finite Coxeter group through the monodromy of a flat connection with values in $\mathfrak{H}$ [13, Sections 1.1 and 1.2]. We note that, whereas Cherednik's affine KZ connection is differential and not abelian, our equations are difference and abelian equations. It seems an interesting problem to relate these two approaches.

1.17. Outline of the paper. In Section 2 we review the definitions of the Yangian $Y_{\hbar}(\mathfrak{g})$ and quantum loop algebra $U_{q}(L \mathfrak{g})$ associated to a symmetrizable Kac-Moody algebra $\mathfrak{g}$.

In Section 3 , we consider the categories $\mathcal{O}_{\text {int }}\left(Y_{\hbar}(\mathfrak{g})\right)$ and $\mathcal{O}_{\text {int }}\left(U_{q}(L \mathfrak{g})\right)$ of representations whose restriction to $\mathfrak{g}$ and $U_{q} \mathfrak{g}$, respectively, is integrable and in category $\mathcal{O}$. We review the classification of their simple objects and prove that the generating series of the loop generators of $Y_{\hbar}(\mathfrak{g})$ and $U_{q}(L \mathfrak{g})$ act on objects of $\mathcal{O}_{\text {int }}\left(Y_{\hbar}(\mathfrak{g})\right), \mathcal{O}_{\text {int }}\left(U_{q}(L \mathfrak{g})\right)$ as rational functions. We then define the subcategories $\mathcal{O}_{\text {int }}^{\Pi}\left(Y_{\hbar}(\mathfrak{g})\right)$ and $\mathcal{O}_{\text {int }}^{\Omega}\left(U_{q}(L \mathfrak{g})\right)$ in terms of the roots of Drinfel'd polynomials. The main result of this section, Theorem [3.8, gives an alternative characterization of these categories in terms of the poles of the fields $\xi_{i}(u), x_{i}^{ \pm}(u)$ of $Y_{\hbar}(\mathfrak{g})$ (resp. $\Psi_{i}(z), \mathcal{X}_{i}^{ \pm}(z)$ of $\left.U_{q}(L \mathfrak{g})\right)$.

Section 4 gives a self-contained account of the theory of additive difference equations including canonical fundamental solutions, monodromy, and a detailed discussion of the inverse problem for abelian equations.

Section 5 gives the definition of the functor $\Gamma: \mathcal{O}_{\text {int }}\left(Y_{\hbar}(\mathfrak{g})\right) \rightarrow \mathcal{O}_{\text {int }}\left(U_{q}(L \mathfrak{g})\right)$ and the proof that the operators defined in Section 1.12 give an action of $U_{q}(L \mathfrak{g})$ on any category $\mathcal{O}_{\text {int }}\left(Y_{\hbar}(\mathfrak{g})\right)$ representation of $Y_{\hbar}(\mathfrak{g})$. We also prove the compatibility of $\Gamma$ with the shift automorphisms of $Y_{\hbar}(\mathfrak{g})$ and $U_{q}(L \mathfrak{g})$.

Section [6 contains the construction of the inverse functor $\urcorner_{\Pi}: \mathcal{O}_{\text {int }}^{\Omega}\left(U_{q}(L \mathfrak{g})\right)$ $\rightarrow \mathcal{O}_{\text {int }}^{\Pi}\left(Y_{\hbar}(\mathfrak{g})\right)$ and the proof that $\rceil_{\Pi} \circ \Gamma_{\Pi}$ and $\left.\Gamma_{\Pi} \circ\right\urcorner_{\Pi}$ are the identity functors on $\mathcal{O}_{\text {int }}^{\Pi}\left(Y_{\hbar}(\mathfrak{g})\right)$ and $\mathcal{O}_{\text {int }}^{\Omega}\left(U_{q}(L \mathfrak{g})\right)$, respectively.

In Section 7, we review the definition of the $q$-characters of $Y_{\hbar}(\mathfrak{g})$ and $U_{q}(L \mathfrak{g})$, and we show that the functor $\Gamma$ is compatible with these.

\section{YANGIANS AND QUANTUM LOOP ALGEBRAS}

2.1. Let $\mathbf{A}=\left(a_{i j}\right)_{i, j \in \mathbf{I}}$ be a symmetrizable generalized Cartan matrix [36]. Thus, $a_{i i}=2$ for any $i \in \mathbf{I}, a_{i j} \in \mathbb{Z}_{\leq 0}$ for any $i \neq j \in \mathbf{I}$, and there exists a diagonal matrix $D$ with positive integer entries $\left\{d_{i}\right\}_{i \in \mathbf{I}}$ such that $D \mathbf{A}$ is symmetric. We assume that $\left(d_{i}\right)$ are relatively prime.

Let $\left(\mathfrak{h},\left\{\alpha_{i}\right\}_{i \in \mathbf{I}},\left\{\alpha_{i}^{\vee}\right\}_{i \in \mathbf{I}}\right)$ be the unique realization of $\mathbf{A}$. Thus, $\mathfrak{h}$ is a complex vector space of dimension $2|\mathbf{I}|-\operatorname{rank}(\mathbf{A}),\left\{\alpha_{i}\right\}_{i \in \mathbf{I}} \subset \mathfrak{h}^{*}$ and $\left\{\alpha_{i}^{\vee}\right\}_{i \in \mathbf{I}} \subset \mathfrak{h}$ are linearly independent sets, and, for any $i, j \in \mathbf{I}, \alpha_{j}\left(\alpha_{i}^{\vee}\right)=a_{i j}$.

Let $\mathfrak{g}$ be the Kac-Moody algebra associated to $\mathbf{A}$. 
2.2. The Yangian $Y_{\hbar}(\mathfrak{g})$. Let $\hbar \in \mathbb{C}$. The Yangian $Y_{\hbar}(\mathfrak{g})$ is the $\mathbb{C}$-algebra generated by elements $\left\{x_{i, r}^{ \pm}, \xi_{i, r}\right\}_{i \in \mathbf{I}, r \in \mathbb{N}}$ and $h \in \mathfrak{h}$, subject to the following relations:

(Y0) For any $i \in \mathbf{I}$,

$$
\xi_{i, 0}=d_{i} \alpha_{i}^{\vee}
$$

(Y1) For any $i, j \in \mathbf{I}, r, s \in \mathbb{N}$, and $h, h^{\prime} \in \mathfrak{h}$,

$$
\left[\xi_{i, r}, \xi_{j, s}\right]=0, \quad\left[\xi_{i, r}, h\right]=0, \quad\left[h, h^{\prime}\right]=0 .
$$

(Y2) For $h \in \mathfrak{h}, j \in \mathbf{I}$, and $s \in \mathbb{N}$,

$$
\left[h, x_{j, s}^{ \pm}\right]= \pm \alpha_{j}(h) x_{j, s}^{ \pm} .
$$

(Y3) For $i, j \in \mathbf{I}$ and $r, s \in \mathbb{N}$,

$$
\left[\xi_{i, r+1}, x_{j, s}^{ \pm}\right]-\left[\xi_{i, r}, x_{j, s+1}^{ \pm}\right]= \pm \hbar \frac{d_{i} a_{i j}}{2}\left(\xi_{i, r} x_{j, s}^{ \pm}+x_{j, s}^{ \pm} \xi_{i, r}\right) .
$$

(Y4) For $i, j \in \mathbf{I}$ and $r, s \in \mathbb{N}$,

$$
\left[x_{i, r+1}^{ \pm}, x_{j, s}^{ \pm}\right]-\left[x_{i, r}^{ \pm}, x_{j, s+1}^{ \pm}\right]= \pm \hbar \frac{d_{i} a_{i j}}{2}\left(x_{i, r}^{ \pm} x_{j, s}^{ \pm}+x_{j, s}^{ \pm} x_{i, r}^{ \pm}\right) .
$$

(Y5) For $i, j \in \mathbf{I}$ and $r, s \in \mathbb{N}$,

$$
\left[x_{i, r}^{+}, x_{j, s}^{-}\right]=\delta_{i j} \xi_{i, r+s} .
$$

(Y6) Let $i \neq j \in \mathbf{I}$, and set $m=1-a_{i j}$. For any $r_{1}, \ldots, r_{m} \in \mathbb{N}$ and $s \in \mathbb{N}$,

$$
\sum_{\pi \in \mathfrak{S}_{m}}\left[x_{i, r_{\pi(1)}}^{ \pm},\left[x_{i, r_{\pi(2)}}^{ \pm},\left[\cdots,\left[x_{i, r_{\pi(m)}}^{ \pm}, x_{j, s}^{ \pm}\right] \cdots\right]\right]=0 .\right.
$$

2.3. Assume now that $\hbar \neq 0$, and define $\xi_{i}(u), x_{i}^{ \pm}(u) \in Y_{\hbar}(\mathfrak{g})\left[\left[u^{-1}\right]\right]$ by

$$
\xi_{i}(u)=1+\hbar \sum_{r \geq 0} \xi_{i, r} u^{-r-1} \quad \text { and } \quad x_{i}^{ \pm}(u)=\hbar \sum_{r \geq 0} x_{i, r}^{ \pm} u^{-r-1} .
$$

Proposition. The relations (Y1),(Y2),(Y3),(Y4),(Y5),(Y6) are equivalent to the following identities in $\left.Y_{\hbar}(\mathfrak{g})\left[u, v ; u^{-1}, v^{-1}\right]\right]$ :

$(\mathcal{Y} 1)$ For any $i, j \in \mathbf{I}$ and $h, h^{\prime} \in \mathfrak{h}$,

$$
\left[\xi_{i}(u), \xi_{j}(v)\right]=0, \quad\left[\xi_{i}(u), h\right]=0, \quad\left[h, h^{\prime}\right]=0 .
$$

$(\mathcal{Y} 2)$ For any $i \in \mathbf{I}$ and $h \in \mathfrak{h}$,

$$
\left[h, x_{i}^{ \pm}(u)\right]= \pm \alpha_{i}(h) x_{i}^{ \pm}(u) .
$$

(Y) For any $i, j \in \mathbf{I}$ and $a=\hbar d_{i} a_{i j} / 2$,

$$
(u-v \mp a) \xi_{i}(u) x_{j}^{ \pm}(v)=(u-v \pm a) x_{j}^{ \pm}(v) \xi_{i}(u) \mp 2 a x_{j}^{ \pm}(u \mp a) \xi_{i}(u) .
$$

$(\mathcal{Y} 4)$ For any $i, j \in \mathbf{I}$ and $a=\hbar d_{i} a_{i j} / 2$.

$$
\begin{aligned}
(u-v \mp a) x_{i}^{ \pm}(u) x_{j}^{ \pm}(v) & \\
& =(u-v \pm a) x_{j}^{ \pm}(v) x_{i}^{ \pm}(u)+\hbar\left(\left[x_{i, 0}^{ \pm}, x_{j}^{ \pm}(v)\right]-\left[x_{i}^{ \pm}(u), x_{j, 0}^{ \pm}\right]\right) .
\end{aligned}
$$

$(\mathcal{Y} 5)$ For any $i, j \in \mathbf{I}$,

$$
(u-v)\left[x_{i}^{+}(u), x_{j}^{-}(v)\right]=-\delta_{i j} \hbar\left(\xi_{i}(u)-\xi_{i}(v)\right) .
$$

(Y) For any $i \neq j \in \mathbf{I}, m=1-a_{i j}$,

$$
\sum_{\pi \in \mathfrak{S}_{m}}\left[x_{i}^{ \pm}\left(u_{\pi(1)}\right),\left[x_{i}^{ \pm}\left(u_{\pi(2)}\right),\left[\cdots\left[x_{i}^{ \pm}\left(u_{\pi(m)}\right), x_{j}^{ \pm}(v)\right] \cdots\right]\right]=0 .\right.
$$


Remark. When $i=j,(\mathcal{Y} 4)$ may be rewritten as follows. Taking $u=v$ yields

$$
\left[x_{i, 0}^{ \pm}, x_{i}^{ \pm}(u)\right]=\mp d_{i} x_{i}^{ \pm}(u)^{2},
$$

and therefore the relation $\left(\mathcal{Y} 4^{\prime}\right)$

$$
\begin{aligned}
&\left(u-v \mp \hbar d_{i}\right) x_{i}^{ \pm}(u) x_{i}^{ \pm}(v) \\
&=\left(u-v \pm \hbar d_{i}\right) x_{i}^{ \pm}(v) x_{i}^{ \pm}(u) \mp \hbar d_{i}\left(x_{i}^{ \pm}(u)^{2}+x_{i}^{ \pm}(v)^{2}\right) .
\end{aligned}
$$

Conversely, equating coefficients of $v^{0}$ in this identity yields (2.1), and therefore $(\mathcal{Y} 4)$.

2.4. Proof of Proposition 2.3. The equivalences $(\mathrm{Y} 1) \equiv(\mathcal{Y} 1)$ and $(\mathrm{Y} 6) \equiv(\mathcal{Y} 6)$ are clear.

To see that (Y4) implies (Y4), multiply (Y4) by $\hbar u^{-r-1} \hbar v^{-s-1}$. Summing over $r, s \geq 0$, and using $\hbar \sum_{r \geq 0} x_{i, r+1}^{ \pm} u^{-r-1}=u x_{i}^{ \pm}(u)-\hbar x_{i, 0}^{ \pm}$yields

$$
\begin{aligned}
(u-v)\left[x_{i}^{ \pm}(u), x_{j}^{ \pm}(v)\right]-\hbar\left(\left[x_{i, 0}^{ \pm}, x_{j}^{ \pm}(v)\right]-\left[x_{i}^{ \pm}(u), x_{j, 0}^{ \pm}\right]\right) & \\
& = \pm a\left(x_{i}^{ \pm}(u) x_{j}^{ \pm}(v)+x_{j}^{ \pm}(v) x_{i}^{ \pm}(u)\right)
\end{aligned}
$$

as claimed. Conversely, taking coefficients of $u^{-r-1} v^{-s-1}$ in $(\mathcal{Y} 4)$ for $r, s \geq 0$ yields $(\mathrm{Y} 4) \sqrt[3]{3}$

(Y2) is clearly equivalent to $(\mathcal{Y} 2)$. To see that (Y2) and (Y3) imply (Y)3), set $\xi_{i,-1}=\hbar^{-1}$ so that

$$
\xi_{i}(u)=\hbar \sum_{r \geq-1} \xi_{i, r} u^{-r-1}
$$

and (Y2) for $h=\xi_{i, 0}$ coincides with (Y3) for $r=-1$. Multiplying both sides of (Y3) by $\hbar u^{-r-1} \hbar v^{-s-1}$ and summing over $r \geq-1, s \geq 0$ yield

$$
(u-v \mp a) \xi_{i}(u) x_{j}^{ \pm}(v)-(u-v \pm a) x_{j}^{ \pm}(v) \xi_{i}(u)=-\hbar\left[\xi_{i}(u), x_{j, 0}^{ \pm}\right] .
$$

Since the right-hand side is independent of $v$, so is the left-hand side. The latter is therefore equal to its value when $v=u \mp a$; that is, $\mp 2 a x_{j}^{ \pm}(u \mp a) \xi_{i}(u)$ and $(\mathcal{Y} 3)$ follow. Conversely, taking coefficients of $u^{-r-1} v^{-s-1}, r, s \geq 0$ in (Y) 3 ) yields (Y3) 4

To see that (Y5) implies (Y5), multiply (Y5) by $\hbar u^{-r-1} \hbar v^{-s-1}$. Summing over $r, s \geq 0$ and using

$$
(u-v) \sum_{r+s=t} u^{-r-1} v^{-s-1}=v^{-t-1}-u^{-t-1}
$$

then yield $(\mathcal{Y} 5)$. Conversely, the above identity implies that $\xi_{i}(u)-\xi_{i}(v)=\hbar(v-$ $u) \sum_{r, s>0} u^{-r-1} v^{-s-1} \xi_{i, r+s}$. Equating this to $\hbar^{-1}(v-u)\left[x_{i}^{+}(u), x_{i}^{-}(v)\right]$ yields (Y5) since $\left.\mathbb{C}\left[u, v ; u^{-1}, v^{-1}\right]\right]$ has no zero divisors.

\footnotetext{
${ }^{3}$ Equating coefficients of $u^{0} v^{-s-1}$ and of $u^{-r-1} v^{0}$ yields the tautological identities $\left[x_{i, 0}^{ \pm}, x_{j, s}^{ \pm}\right]=$ $\left[x_{i, 0}^{ \pm}, x_{j, s}^{ \pm}\right]$and $\left[x_{i, r}^{ \pm}, x_{j, 0}^{ \pm}\right]=\left[x_{i, r}^{ \pm}, x_{j, 0}^{ \pm}\right]$, respectively.

${ }^{4}$ Equating coefficients of $u^{1}, u^{0}$ yields the identities $x_{j}^{ \pm}(v)=x_{j}^{ \pm}(v)$ and (Y2) for $h=\xi_{i, 0}$, respectively, while equating coefficients of $v^{0}$ yields the identity $-\hbar\left[\xi_{i}(u), x_{j, 0}^{ \pm}\right]=\mp 2 a x_{j}^{ \pm}(u \mp$ a) $\xi_{i}(u)$.
} 
2.5. The following result is due to Levendorskil [39]. We reproduce his proof below for completeness.

Lemma. The relation (Y6) follows from (Y1)-(Y3) and the special case of (Y6) when $r_{1}=\cdots=r_{m}=0$.

Proof. The proof of this lemma is based on the construction of commuting elements $\left\{\widetilde{t}_{i, r}\right\}_{i \in \mathbf{I}, r \in \mathbb{N}}$. These are polynomials in $\left\{\xi_{i, r}\right\}$ such that the following commutation relations hold:

$$
\begin{aligned}
{\left[\widetilde{t}_{i, r}, x_{i, s}^{ \pm}\right] } & = \pm 2 d_{i} x_{i, r+s}^{ \pm}, \\
{\left[\widetilde{t}_{i, r}, x_{j, s}^{ \pm}\right] } & =\sum_{k=0}^{r+s} b_{i j}^{ \pm}(r, s, k) x_{j, r+s-k}^{ \pm},
\end{aligned}
$$

where $b_{i j}^{ \pm}(r, s, k) \in \mathbb{C}$ are scalars. We postpone their construction to 2.6 and proceed with the proof of the lemma.

Denote the left-hand side of $(\mathrm{Y} 6)$ by $S_{i j}^{ \pm}\left(r_{1}, \ldots, r_{m} ; s\right)$. We prove that this element is zero by induction on the number of non-zero $r_{j}$. The case $S_{i j}^{ \pm}(0, \ldots, 0 ; s)=0$ holds by assumption. Assume now that

$$
S_{i j}^{ \pm}\left(r_{1}, \ldots, r_{k}, 0, \ldots, 0 ; s\right)=0
$$

for some $0 \leq k<m$ and for every $r_{1}, \ldots, r_{k}, s \in \mathbb{N}$. Applying $\operatorname{ad}\left(\widetilde{t}_{i, r}\right)$ to this identity yields

$$
\begin{aligned}
0= & \pm 2 d_{i}(m-k) S_{i j}^{ \pm}\left(r_{1}, \ldots, r_{k}, r, 0, \ldots, 0 ; s\right) \\
& \pm 2 d_{i} \sum_{p=1}^{k} S_{i j}^{ \pm}\left(r_{1}, \ldots, r_{p-1}, r_{p}+r, r_{p+1}, \ldots, r_{k}, 0, \ldots, 0 ; s\right) \\
& +\sum_{p=0}^{r+s} b_{i j}^{ \pm}(r, s, p) S_{i j}^{ \pm}\left(r_{1}, \ldots, r_{k}, 0, \ldots, 0 ; r+s-p\right) .
\end{aligned}
$$

Since the terms in the last two sums are zero by induction, it follows that $S_{i j}^{ \pm}\left(r_{1}, \ldots\right.$, $\left.r_{k}, r, 0, \ldots, 0 ; s\right)=0$.

2.6. Define $\left\{t_{i, r}\right\}_{i \in \mathbf{I}, r \in \mathbb{N}}$ by

$$
\hbar \sum_{r \geq 0} t_{i, r} u^{-r-1}=\log \left(\xi_{i}(u)\right) .
$$

The following commutation relation was obtained in [39, Lemma 1.9] (see also [26, Remark 2.9]):

$$
\left[t_{i, r}, x_{j, s}^{ \pm}\right]= \pm d_{i} a_{i j} \sum_{l=0}^{\lfloor r / 2\rfloor}\left(\begin{array}{c}
r \\
2 l
\end{array}\right) \frac{\left(\hbar d_{i} a_{i j} / 2\right)^{2 l}}{2 l+1} x_{j, r+s-2 l}^{ \pm} .
$$

Now it is clear that one can define $\widetilde{t}_{i, r}$ as

$$
\widetilde{t}_{i, r}=t_{i, r}+\text { linear combination of } t_{i, r-k}(1 \leq k \leq r)
$$

such that $\left[\tilde{t}_{i, r}, x_{i, s}^{ \pm}\right]= \pm 2 d_{i} x_{i, r+s}^{ \pm}$. The commutation relation above, and the lower triangularity of the transformation matrix between $\left\{\widetilde{t}_{i, r}\right\}$ and $\left\{t_{i, r}\right\}$ implies that we 
also have

$$
\left[\widetilde{t}_{i, r}, x_{j, s}^{ \pm}\right]=\sum_{p=0}^{r+s} b_{i j}^{ \pm}(r, s, p) x_{j, r+s-p}^{ \pm}
$$

for some scalars $b_{i j}^{ \pm}(r, s, p)$.

2.7. It follows from Lemma 2.5 that, on an integrable representation, relation (Y6) can be deduced from the other relations. More precisely, let $\widetilde{Y}_{\hbar}(\mathfrak{g})$ be a unital associative algebra generated by $\left\{h, \xi_{i, r}, x_{i, r}^{ \pm}\right\}$subject to the relations (Y0)-(Y5) of Section 2.2. Let $V$ be a representation of $\widetilde{Y}_{\hbar}(\mathfrak{g})$ which is integrable in the following sense:

- $V$ is $\mathfrak{h}$-diagonalizable with finite-dimensional weight spaces.

- For each $i \in \mathbf{I}$, the action of $x_{i, 0}^{ \pm}$on $V$ is locally nilpotent.

Proposition. The action of $\widetilde{Y}_{\hbar}(\mathfrak{g})$ on $V$ factors through one of $Y_{\hbar}(\mathfrak{g})$.

Proof. By Lemma 2.5, it suffices to prove that relation (Y6) with $r_{1}=\cdots=r_{m}=0$ holds in $\operatorname{End}(V)$. Let $\mathfrak{s i}_{2}^{i} \subset Y_{\hbar}(\mathfrak{g})$ be the Lie subalgebra generated by $\left\{\xi_{i, 0}, x_{i, 0}^{ \pm}\right\}$. Let $V[\mu], \mu \in \mathfrak{h}^{*}$ be a weight subspace of $V$, and set

$$
\bar{V}=\bigoplus_{m \in \mathbb{Z}} V\left[\mu+m \alpha_{i}\right] \quad \text { and } \quad \bar{V}_{j}^{ \pm}=\bigoplus_{m \in \mathbb{Z}} V\left[\mu+m \alpha_{i} \pm \alpha_{j}\right] .
$$

$\bar{V}, \bar{V}_{j}^{ \pm}$are $\mathfrak{s l}_{2}^{i}$-invariant subspaces of $V$ which, by the integrability of $V$, are finitedimensional. Since $\left[\xi_{i, 0}, x_{j, s}^{ \pm}\right]= \pm d_{i} a_{i j} x_{j, s}^{ \pm}$and $\operatorname{ad}\left(x_{i, 0}^{\mp}\right) x_{j, s}^{ \pm}=0$, the restriction of $x_{j, s}^{ \pm}$to $\bar{V}$ is the lowest (resp. highest) weight vector in the finite-dimensional $\mathfrak{s i}_{2}^{i}$-module $\operatorname{Hom}\left(\bar{V}, \bar{V}_{j}^{ \pm}\right)$, which implies that $\operatorname{ad}\left(x_{i, 0}^{ \pm}\right)^{1-a_{i j}} x_{j, s}^{ \pm}$acts by 0 on $\bar{V}$.

2.8. Shift automorphism. The group of translations of the complex plane acts on $Y_{\hbar}(\mathfrak{g})$ by

$$
\tau_{a}\left(y_{r}\right)=\sum_{s=0}^{r}\left(\begin{array}{c}
r \\
s
\end{array}\right) a^{r-s} y_{s} \quad \text { and } \quad \tau_{a}(h)=h,
$$

where $a \in \mathbb{C}, y$ is one of $\xi_{i}, x_{i}^{ \pm}, r \in \mathbb{N}$, and $h \in \mathfrak{h}$. In terms of the generating series introduced in 2.3 .

$$
\tau_{a}(y(u))=y(u-a) .
$$

Given a representation $V$ of $Y_{\hbar}(\mathfrak{g})$ and $a \in \mathbb{C}$, we set $V(a)=\tau_{a}^{*}(V)$.

2.9. The quantum loop algebra $U_{q}(L \mathfrak{g})$. Let $q \in \mathbb{C}^{\times}$be of infinite order. For any $i \in \mathbf{I}$, set $q_{i}=q^{d_{i}}$. We use the standard notation for Gaussian integers

$$
\begin{gathered}
{[n]_{q}=\frac{q^{n}-q^{-n}}{q-q^{-1}}} \\
{[n]_{q} !=[n]_{q}[n-1]_{q} \cdots[1]_{q}, \quad\left[\begin{array}{c}
n \\
k
\end{array}\right]_{q}=\frac{[n]_{q} !}{[k]_{q} ![n-k]_{q} !} .}
\end{gathered}
$$

The quantum loop algebra $U_{q}(L \mathfrak{g})$ is the $\mathbb{C}$-algebra generated by elements

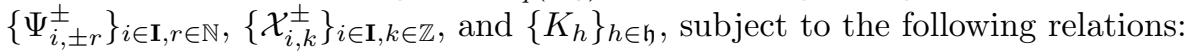

(QL0) For any $i \in \mathbf{I}$,

$$
\Psi_{i, 0}^{ \pm}=K_{ \pm d_{i} \alpha_{i}^{\vee}}
$$


(QL1) For any $i, j \in \mathbf{I}, r, s \in \mathbb{N}$, and $h, h^{\prime} \in \mathfrak{h}$,

$$
\begin{gathered}
{\left[\Psi_{i, \pm r}^{ \pm}, \Psi_{j, \pm s}^{ \pm}\right]=0, \quad\left[\Psi_{i, \pm r}^{ \pm}, \Psi_{j, \mp s}^{\mp}\right]=0, \quad\left[\Psi_{i, \pm r}^{ \pm}, K_{h}\right]=0,} \\
K_{h} K_{h^{\prime}}=K_{h+h^{\prime}}, \quad K_{0}=1 .
\end{gathered}
$$

(QL2) For any $i \in \mathbf{I}, k \in \mathbb{Z}$, and $h \in \mathfrak{h}$,

$$
K_{h} \mathcal{X}_{i, k}^{ \pm} K_{h}^{-1}=q^{ \pm \alpha_{i}(h)} \mathcal{X}_{i, k}^{ \pm}
$$

(QL3) For any $i, j \in \mathbf{I}, \varepsilon \in\{ \pm\}$, and $l \in \mathbb{Z}$,

$$
\Psi_{i, k+1}^{\varepsilon} \mathcal{X}_{j, l}^{ \pm}-q_{i}^{ \pm a_{i j}} \mathcal{X}_{j, l}^{ \pm} \Psi_{i, k+1}^{\varepsilon}=q_{i}^{ \pm a_{i j}} \Psi_{i, k}^{\varepsilon} \mathcal{X}_{j, l+1}^{ \pm}-\mathcal{X}_{j, l+1}^{ \pm} \Psi_{i, k}^{\varepsilon},
$$

for any $k \in \mathbb{Z}_{\geq 0}$ if $\varepsilon=+$ and $k \in \mathbb{Z}_{<0}$ if $\varepsilon=-$.

(QL4) For any $i, j \in \mathbf{I}$ and $k, l \in \mathbb{Z}$,

$$
\mathcal{X}_{i, k+1}^{ \pm} \mathcal{X}_{j, l}^{ \pm}-q_{i}^{ \pm a_{i j}} \mathcal{X}_{j, l}^{ \pm} \mathcal{X}_{i, k+1}^{ \pm}=q_{i}^{ \pm a_{i j}} \mathcal{X}_{i, k}^{ \pm} \mathcal{X}_{j, l+1}^{ \pm}-\mathcal{X}_{j, l+1}^{ \pm} \mathcal{X}_{i, k}^{ \pm}
$$

(QL5) For any $i, j \in \mathbf{I}$ and $k, l \in \mathbb{Z}$,

$$
\left[\mathcal{X}_{i, k}^{+}, \mathcal{X}_{j, l}^{-}\right]=\delta_{i j} \frac{\Psi_{i, k+l}^{+}-\Psi_{i, k+l}^{-}}{q_{i}-q_{i}^{-1}},
$$

where we set $\Psi_{i, \mp k}^{ \pm}=0$ for any $k \geq 1$.

(QL6) For any $i \neq j \in \mathbf{I}, m=1-a_{i j}, k_{1}, \ldots, k_{m} \in \mathbb{Z}$, and $l \in \mathbb{Z}$,

$$
\sum_{\pi \in \mathfrak{S}_{m}} \sum_{s=0}^{m}(-1)^{s}\left[\begin{array}{c}
m \\
s
\end{array}\right]_{q_{i}} \mathcal{X}_{i, k_{\pi(1)}}^{ \pm} \cdots \mathcal{X}_{i, k_{\pi(s)}}^{ \pm} \mathcal{X}_{j, l}^{ \pm} \mathcal{X}_{i, k_{\pi(s+1)}}^{ \pm} \cdots \mathcal{X}_{i, k_{\pi(m)}}^{ \pm}=0 .
$$

2.10. Define $\Psi_{i}(z)^{+}, \mathcal{X}_{i}^{ \pm}(z)^{+} \in U_{q}(L \mathfrak{g})\left[\left[z^{-1}\right]\right]$ and $\Psi_{i}(z)^{-}, \mathcal{X}_{i}^{ \pm}(z)^{-} \in U_{q}(L \mathfrak{g})[[z]]$ by

$$
\begin{aligned}
\Psi_{i}(z)^{+} & =\sum_{r \geq 0} \Psi_{i, r}^{+} z^{-r}, & \Psi_{i}(z)^{-} & =\sum_{r \leq 0} \Psi_{i, r}^{-} z^{-r}, \\
\mathcal{X}_{i}^{ \pm}(z)^{+} & =\sum_{r \geq 0} \mathcal{X}_{i, r}^{ \pm} z^{-r}, & \mathcal{X}_{i}^{ \pm}(z)^{-} & =-\sum_{r<0} \mathcal{X}_{i, r}^{ \pm} z^{-r} .
\end{aligned}
$$

Proposition. The relations (QL1),(QL2),(QL3),(QL4),(QL5),(QL6) imply the following relations in $\left.U_{q}(L \mathfrak{g})\left[z, w ; z^{-1}, w^{-1}\right]\right]$ :

$(\mathcal{Q L} 1)$ For any $i, j \in \mathbf{I}$ and $h, h^{\prime} \in \mathfrak{h}$,

$$
\begin{array}{cc}
{\left[\Psi_{i}(z)^{+}, \Psi_{j}(w)^{+}\right]=0,} & {\left[\Psi_{i}(z)^{+}, K_{h}\right]=0,} \\
K_{h} K_{h^{\prime}}=K_{h+h^{\prime}}, & K_{0}=1 .
\end{array}
$$

$(\mathcal{Q} \mathcal{L} 2)$ For any $i \in \mathbf{I}$ and $h \in \mathfrak{h}$,

$$
K_{h} \mathcal{X}_{i}^{ \pm}(z)^{+} K_{h}^{-1}=q^{ \pm \alpha_{i}(h)} \mathcal{X}_{i}^{ \pm}(z)^{+} .
$$

$(\mathcal{Q L} 3)$ For any $i, j \in \mathbf{I}$,

$$
\begin{aligned}
& \left(z-q_{i}^{ \pm a_{i j}} w\right) \Psi_{i}(z)^{+} \mathcal{X}_{j}^{ \pm}(w)^{+} \\
& \quad=\left(q_{i}^{ \pm a_{i j}} z-w\right) \mathcal{X}_{j}^{ \pm}(w)^{+} \Psi_{i}(z)^{+}-\left(q_{i}^{ \pm a_{i j}}-q_{i}^{\mp a_{i j}}\right) q_{i}^{ \pm a_{i j}} w \mathcal{X}_{j}^{ \pm}\left(q_{i}^{\mp a_{i j}} z\right)^{+} \Psi_{i}(z)^{+} .
\end{aligned}
$$

$(\mathcal{Q L} 4)$ For any $i, j \in \mathbf{I}$,

$$
\begin{aligned}
& \left(z-q_{i}^{ \pm a_{i j}} w\right) \mathcal{X}_{i}^{ \pm}(z)^{+} \mathcal{X}_{j}^{ \pm}(w)^{+}-\left(q_{i}^{ \pm a_{i j}} z-w\right) \mathcal{X}_{j}^{ \pm}(w)^{+} \mathcal{X}_{i}^{ \pm}(z)^{+} \\
= & z\left(\mathcal{X}_{i, 0}^{ \pm} \mathcal{X}_{j}^{ \pm}(w)^{+}-q_{i}^{ \pm a_{i j}} \mathcal{X}_{j}^{ \pm}(w)^{+} \mathcal{X}_{i, 0}^{ \pm}\right)+w\left(\mathcal{X}_{j, 0}^{ \pm} \mathcal{X}_{i}^{ \pm}(z)^{+}-q_{i}^{ \pm a_{i j}} \mathcal{X}_{i}^{ \pm}(z)^{+} \mathcal{X}_{j, 0}^{ \pm}\right)
\end{aligned}
$$


$(\mathcal{Q L} 5)$ For any $i, j \in \mathbf{I}$,

$$
(z-w)\left[\mathcal{X}_{i}^{+}(z)^{+}, \mathcal{X}_{j}^{-}(w)^{+}\right]=\frac{\delta_{i j}}{q_{i}-q_{i}^{-1}}\left(z \Psi_{i}(w)^{+}-w \Psi_{i}(z)^{+}-(z-w) \Psi_{i, 0}^{-}\right) .
$$

$(\mathcal{Q L} 6)$ For any $i \neq j \in \mathbf{I}$ and $m=1-a_{i j}$,

$$
\begin{aligned}
\sum_{\pi \in \mathfrak{S}_{m}} \sum_{s=0}^{m}(-1)^{s}\left[\begin{array}{c}
m \\
s
\end{array}\right]_{q_{i}} \mathcal{X}_{i}^{ \pm}\left(z_{\pi(1)}\right)^{+} \ldots \mathcal{X}_{i}^{ \pm}\left(z_{\pi(s)}\right)^{+} \mathcal{X}_{j}^{ \pm}(w)^{+} \\
\cdot \mathcal{X}_{i}^{ \pm}\left(z_{\pi(s+1)}\right)^{+} \ldots \mathcal{X}_{i}^{ \pm}\left(z_{\pi(m)}\right)^{+}=0 .
\end{aligned}
$$

Remark. When $i=j,(\mathcal{Q L} 4)$ may be rewritten as follows. Taking $z=w$ yields

$$
\left(\mathcal{X}_{i, 0}^{ \pm} \mathcal{X}_{i}^{ \pm}(z)-q_{i}^{ \pm 2} \mathcal{X}_{i}^{ \pm}(z) \mathcal{X}_{i, 0}^{ \pm}\right)=\left(1-q_{i}^{ \pm 2}\right) \mathcal{X}_{i}^{ \pm}(z)^{2},
$$

and therefore the relation $\left(\mathcal{Q L} 4^{\prime}\right)$

$$
\begin{aligned}
\left(z-q_{i}^{ \pm 2} w\right) \mathcal{X}_{i}^{ \pm}(z) \mathcal{X}_{i}^{ \pm}(w)-\left(q_{i}^{ \pm 2} z-w\right) \mathcal{X}_{i}^{ \pm}(w) \mathcal{X}_{i}^{ \pm}(z) & \\
& =z\left(1-q_{i}^{ \pm 2}\right) \mathcal{X}_{i}^{ \pm}(w)^{2}+w\left(1-q_{i}^{ \pm 2}\right) \mathcal{X}_{i}^{ \pm}(z)^{2}
\end{aligned}
$$

Conversely, equating coefficients of $z$ in this identity yields (2.2) and therefore $(\mathcal{Q L} 4)$.

2.11. Proof of Proposition 2.10. The implications $(\mathrm{QL} n) \Rightarrow(\mathcal{Q L} n)$, for $n=$ $1,2,6$ are clear.

Multiplying (QL4) by $z^{-k} w^{-l}$, summing over $k, l \geq 0$, and using the fact that $\sum_{k \geq 0} \mathcal{X}_{i, k+1}^{ \pm} z^{-k}=z\left(\mathcal{X}_{i}^{ \pm}(z)-\mathcal{X}_{i, 0}^{ \pm}\right)$yield $(\mathcal{Q L} 4)$.

Multiplying (QL3) by $z^{-k} w^{-l}$ and summing over $k, l \geq 0$ yield similarly

$$
\begin{aligned}
& \left(z-q_{i}^{ \pm a_{i j}} w\right) \Psi_{i}(z)^{+} \mathcal{X}_{j}^{ \pm}(w)^{+}-\left(q_{i}^{ \pm a_{i j}} z-w\right) \mathcal{X}_{j}^{ \pm}(w)^{+} \Psi_{i}(z)^{+} \\
& \quad=z\left(\Psi_{i, 0}^{+} \mathcal{X}_{j}^{ \pm}(w)^{+}-q_{i}^{ \pm a_{i j}} \mathcal{X}_{j}^{ \pm}(w)^{+} \Psi_{i, 0}^{+}\right)-w\left(q_{i}^{ \pm a_{i j}} \Psi_{i}(z)^{+} \mathcal{X}_{j, 0}^{ \pm}-\mathcal{X}_{j, 0}^{ \pm} \Psi_{i}(z)^{+}\right) .
\end{aligned}
$$

Since the first summand on the right-hand side is equal to 0 by (QL2), the ratio of the left-hand side by $w$ is independent of $w$. It is therefore equal to its value for $w=q_{i}^{\mp a_{i j}} z$; that is, $-q_{i}^{ \pm a_{i j}}\left(q_{i}^{ \pm a_{i j}}-q_{i}^{\mp a_{i j}}\right) \mathcal{X}_{j}^{ \pm}\left(q_{i}^{\mp a_{i j}} z\right)^{+} \Psi_{i}(z)^{+}$and $(\mathcal{Q L} 3)$ holds.

Multiplying (QL5) by $(z-w) z^{-k} w^{-l}$, summing over $k, l \geq 0$, and using $(z-$ $w) \sum_{k+l=m} z^{-k} w^{-l}=z w^{-m}-w z^{-m}$ yield $(\mathcal{Q L} 5)$.

Remark. The relations $(\mathcal{Q L} 1)-(\mathcal{Q L} 6)$ of Proposition 2.10 for $\left\{\Psi_{i}(z)^{-}, \mathcal{X}_{i}^{ \pm}(z)^{-}\right\}$ continue to hold in $U_{q}(L \mathfrak{g})[[z, w]]$. Similarly these relations remain true between + and - fields, in the appropriate algebra. For example, $(\mathcal{Q L} 3)$ for $\Psi_{i}(z)^{+}$and $\mathcal{X}_{i}^{ \pm}(w)^{-}$is true in $\left.U_{q}(L \mathfrak{g})\left[z, w^{-1} ; z^{-1}, w\right]\right]$.

2.12. The following result is the analogue of Lemma 2.5 for $U_{q}(L \mathfrak{g})$.

Lemma. The relation (QL6) follows from (QL1)-(QL3) and the special case of (QL6) when $k_{1}=\cdots=k_{m}=0$.

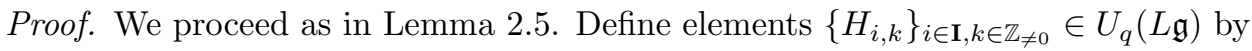

$$
\Psi_{i}(z)^{ \pm}=\Psi_{i, 0}^{ \pm} \exp \left( \pm\left(q_{i}-q_{i}^{-1}\right) \sum_{n \geq 1} H_{i, \pm n} z^{\mp n}\right) .
$$


Then we have the following relation:

$$
\left[H_{i, k}, \mathcal{X}_{j, l}^{ \pm}\right]= \pm \frac{\left[k a_{i j}\right]_{q_{i}}}{k} \mathcal{X}_{j, k+l}^{ \pm}
$$

The proof then proceeds by an induction argument, as in that of Lemma 2.5.

2.13. Let $\widetilde{U}_{q}(L \mathfrak{g})$ be the unital associative algebra generated by elements $\left\{K_{h}, \Psi_{i, \pm r}^{ \pm}, \mathcal{X}_{i, l}^{ \pm}\right\}$subject to the relations (QL0)-(QL5) of Section 2.9. Let $\mathcal{V}$ be a representation of $\widetilde{U}_{q}(L \mathfrak{g})$ which is integrable in the following sense:

- $\mathcal{V}$ is $\mathfrak{h}$-diagonalizable with finite-dimensional weight spaces.

- For each $i \in \mathbf{I}$, the action of $\mathcal{X}_{i, 0}^{ \pm}$on $\mathcal{V}$ is locally nilpotent.

The proof of the following result is similar to that of Proposition 2.7 and therefore is omitted.

Proposition. The action of $\widetilde{U}_{q}(L \mathfrak{g})$ on $\mathcal{V}$ descends to that of $U_{q}(L \mathfrak{g})$.

2.14. Shift automorphism. The group $\mathbb{C}^{*}$ of dilations of the complex plane acts on $U_{q}(L \mathfrak{g})$ by

$$
\tau_{\alpha}\left(Y_{k}\right)=\alpha^{k} Y_{k} \quad \text { and } \quad \tau_{\alpha}\left(K_{h}\right)=K_{h},
$$

where $\alpha \in \mathbb{C}^{*}, Y$ is one of $\Psi_{i}^{ \pm}, \mathcal{X}_{i}^{ \pm}, k \in \mathbb{Z}$, and $h \in \mathfrak{h}$. In terms of the generating series of 2.10, we have

$$
\tau_{\alpha}\left(Y(z)^{ \pm}\right)=Y\left(\alpha^{-1} z\right)^{ \pm} .
$$

Given a representation $\mathcal{V}$ of $U_{q}(L \mathfrak{g})$ and $\alpha \in \mathbb{C}^{\times}$, we denote $\tau_{\alpha}^{*}(\mathcal{V})$ by $\mathcal{V}(\alpha)$.

\section{Integrable REPRESEntations of $Y_{\hbar}(\mathfrak{g})$ AND $U_{q}(L \mathfrak{g})$}

3.1. Integrable representations [32]. By definition, a representation of $U_{q}(L \mathfrak{g})$ $\left(\right.$ resp. $\left.Y_{\hbar}(\mathfrak{g})\right)$ is in $\mathcal{O}_{\text {int }}\left(U_{q}(L \mathfrak{g})\right)$ (resp. $\left.\mathcal{O}_{\text {int }}\left(Y_{\hbar}(\mathfrak{g})\right)\right)$ if and only if its restriction to $U_{q}(\mathfrak{g})$ (resp. $\mathfrak{g}$ ) is an integrable representation in the corresponding category $\mathcal{O}$ (see [36,41]). Specifically,

(i) A representation $\mathcal{V}$ of $U_{q}(L \mathfrak{g})$ is in $\mathcal{O}_{\text {int }}\left(U_{q}(L \mathfrak{g})\right)$ if

(a) $\mathcal{V}=\bigoplus_{\mu \in \mathfrak{h}^{*}} \mathcal{V}_{\mu}$ and $\operatorname{dim}\left(\mathcal{V}_{\mu}\right)<\infty$, where

$$
\mathcal{V}_{\mu}=\left\{v \in \mathcal{V} \mid K_{h} v=q^{\mu(h)} v \text { for every } h \in \mathfrak{h}\right\} .
$$

(b) There exist $\lambda_{1}, \ldots, \lambda_{r} \in \mathfrak{h}^{*}$ such that $\mathcal{V}_{\mu} \neq 0$ implies that $\mu \leq \lambda_{i}$ for some $i=1, \ldots, r$. Recall that we say $\mu \leq \lambda$ if $\lambda-\mu \in \sum_{i \in \mathbf{I}} \mathbb{N} \alpha_{i}$.

(c) For each $\mu \in \mathfrak{h}^{*}$ such that $\mathcal{V}_{\mu} \neq 0$ and $i \in \mathbf{I}$, there exists $N>0$ such that $\mathcal{V}_{\mu-n \alpha_{i}}=0$ for every $n \geq N$.

(ii) A representation $V$ of $Y_{\hbar}(\mathfrak{g})$ is in $\mathcal{O}_{\text {int }}\left(Y_{\hbar}(\mathfrak{g})\right)$ if

(a) $V=\bigoplus_{\mu \in \mathfrak{h}^{*}} V_{\mu}$ and $\operatorname{dim}\left(V_{\mu}\right)<\infty$, where

$$
V_{\mu}=\{v \in V \mid h v=\mu(h) v \text { for every } h \in \mathfrak{h}\} .
$$

(b) There exist $\lambda_{1}, \ldots, \lambda_{r} \in \mathfrak{h}^{*}$ such that $V_{\mu} \neq 0$ implies that $\mu \leq \lambda_{i}$ for some $i=1, \ldots, r$.

(c) For each $\mu \in \mathfrak{h}^{*}$ such that $V_{\mu} \neq 0$ and $i \in \mathbf{I}$, there exists $N>0$ such that $V_{\mu-n \alpha_{i}}=0$ for every $n \geq N$. 
Remark. When $\mathfrak{g}$ is a semisimple Lie algebra, the categories $\mathcal{O}_{\text {int }}\left(Y_{\hbar}(\mathfrak{g})\right)$ and $\mathcal{O}_{\text {int }}\left(U_{q}(L \mathfrak{g})\right)$ are the categories of finite-dimensional representations of $Y_{\hbar}(\mathfrak{g})$ and $U_{q}(L \mathfrak{g})$. Note that in this case, the definition of an integrable representation in category $\mathcal{O}$ for $U_{q}(L \mathfrak{g})$ given above differs from the usual one for $U_{q} \hat{\mathfrak{g}}$ given in [36, 41.

3.2. Drinfel'd polynomials: Yangians. We recall below the classification of irreducible representations in $\mathcal{O}_{\text {int }}\left(Y_{\hbar}(\mathfrak{g})\right)$. For $\mathfrak{g}=\mathfrak{s l}_{2}$, this result is proved in [9]. For the general case, a proof can be obtained by adapting the arguments of [12]; see [32] and [11, Chapter 12].

Let $\mathbf{c}=\left\{c_{i, r}\right\}_{r \in \mathbb{N}, i \in \mathbf{I}} \subset \mathbb{C}$ be a collection of complex numbers, and let $\lambda \in \mathfrak{h}^{*}$ such that $c_{i, 0}=d_{i} \lambda\left(\alpha_{i}^{\vee}\right)$. A representation $V$ of $Y_{\hbar}(\mathfrak{g})$ is said to be the highest weight representation of highest weight $(\lambda, \mathbf{c})$ if there exists $\mathbf{v} \in V$ such that

(i) $V=Y_{\hbar}(\mathfrak{g}) \mathbf{v}$.

(ii) $x_{i, r}^{+} \mathbf{v}=0$ for every $i \in \mathbf{I}, r \in \mathbb{N}$.

(iii) $\xi_{i, r} \mathbf{v}=c_{i, r} \mathbf{v}$ and $h \mathbf{v}=\lambda(h) \mathbf{v}$, for every $i \in \mathbf{I}, r \in \mathbb{N}$, and $h \in \mathfrak{h}$.

To the pair $(\lambda, \mathbf{c})$, we associate the Verma module $M(\lambda, \mathbf{c})$ in an obvious way and denote by $L(\lambda, \mathbf{c})$ its unique irreducible quotient.

\section{Theorem.}

(i) Every irreducible representation in $\mathcal{O}_{\text {int }}\left(Y_{\hbar}(\mathfrak{g})\right)$ is a highest weight representation for a unique highest weight $(\lambda, \mathbf{c})$.

(ii) The irreducible representation $L(\lambda, \mathbf{c})$ is in $\mathcal{O}_{\text {int }}\left(Y_{\hbar}(\mathfrak{g})\right)$ if and only if there exist (unique) monic polynomials $\left\{P_{i}(u) \in \mathbb{C}[u]\right\}_{i \in \mathbf{I}}$ such that

$$
1+\hbar \sum_{r \geq 0} c_{i, r} u^{-r-1}=\frac{P_{i}\left(u+d_{i} \hbar\right)}{P_{i}(u)} .
$$

It follows from (ii) that $\lambda\left(\alpha_{i}^{\vee}\right)=\operatorname{deg}\left(P_{i}\right) \in \mathbb{N}$ and hence that $\lambda$ is a dominant integral weight. The polynomials $\left\{P_{i}(u)\right\}$ are called Drinfel'd polynomials. By Theorem 3.2 the set of isomorphism classes of simple objects in $\mathcal{O}_{\text {int }}\left(Y_{\hbar}(\mathfrak{g})\right)$ is in bijection with the set $\mathcal{P}_{+}^{Y}$ of pairs $\left(\lambda \in \mathfrak{h}^{*},\left\{P_{i}(u) \in \mathbb{C}[u]\right\}_{i \in \mathbf{I}}\right)$ such that $\lambda\left(\alpha_{i}^{\vee}\right)=\operatorname{deg}\left(P_{i}\right)$ and $P_{i}$ are monic. If $\left(\lambda,\left\{P_{i}\right\}\right) \in \mathcal{P}_{+}^{Y}$, we denote by $L\left(\lambda,\left\{P_{i}\right\}\right)$ the corresponding irreducible $Y_{\hbar}(\mathfrak{g})$-module.

3.3. Drinfel'd polynomials: Quantum loop algebra. Let $\gamma=\left\{\gamma_{i, \pm m}^{ \pm}\right\}_{i \in \mathbf{I}, m \in \mathbb{N}}$ be a collection of complex numbers and $\lambda \in \mathfrak{h}^{*}$ such that $\gamma_{i, 0}^{ \pm}=q_{i}^{ \pm \lambda\left(\alpha_{i}^{\vee}\right)}$. A representation $\mathcal{V}$ of $U_{q}(L \mathfrak{g})$ is said to be the l-highest weight representation of the $l$-highest weight $(\lambda, \gamma)$ if there exists $\mathbf{v} \in \mathcal{V}$ such that

(i) $\mathcal{V}=U_{q}(L \mathfrak{g}) \mathbf{v}$

(ii) $\mathcal{X}_{i, k}^{+} \mathbf{v}=0$ for every $i \in \mathbf{I}$ and $k \in \mathbb{Z}$.

(iii) $\Psi_{i, \pm m}^{ \pm} \mathbf{v}=\gamma_{i, \pm m}^{ \pm} \mathbf{v}$ and $K_{h} \mathbf{v}=q^{\lambda(h)} \mathbf{v}$ for every $i \in \mathbf{I}, m \in \mathbb{N}$, and $h \in \mathfrak{h}$.

As for Yangians, for any $(\lambda, \gamma)$, there is a unique irreducible representation, $\mathcal{L}(\lambda, \gamma)$, with highest weight $(\lambda, \gamma)$.

\section{Theorem.}

(i) Every irreducible representation in $\mathcal{O}_{\text {int }}\left(U_{q}(L \mathfrak{g})\right)$ is the highest weight representation for a unique highest weight $(\lambda, \gamma)$. 
(ii) The irreducible representation $\mathcal{L}(\lambda, \gamma)$ is in $\mathcal{O}_{\text {int }}\left(U_{q}(L \mathfrak{g})\right)$ if and only if there exist monic polynomials $\left\{\mathcal{P}_{i}(w) \in \mathbb{C}[w]\right\}_{i \in \mathbf{I}}, \mathcal{P}_{i}(0) \neq 0$, such that

$$
\sum_{m \geq 0} \gamma_{i, m}^{+} z^{-m}=q_{i}^{-\operatorname{deg}\left(\mathcal{P}_{i}\right)} \frac{\mathcal{P}_{i}\left(q^{2} z\right)}{\mathcal{P}_{i}(z)}=\sum_{m \leq 0} \gamma_{i, m}^{-} z^{m} .
$$

Again, we have $\lambda\left(\alpha_{i}^{\vee}\right)=\operatorname{deg}\left(\mathcal{P}_{i}\right)$, and hence $\lambda$ is a dominant integral weight. The polynomials $\mathcal{P}_{i}$ are again called Drinfel'd polynomials. The set of isomorphism classes of simple objects in $\mathcal{O}_{\text {int }}\left(U_{q}(L \mathfrak{g})\right)$ is in bijection with the set $\mathcal{P}_{+}^{U}$ of pairs $\left(\lambda \in \mathfrak{h}^{*},\left\{\mathcal{P}_{i}\right\}\right)$ such that $\mathcal{P}_{i}$ are monic, $\mathcal{P}_{i}(0) \neq 0$, and $\lambda\left(\alpha_{i}^{\vee}\right)=\operatorname{deg}\left(\mathcal{P}_{i}\right)$. We denote by $\mathcal{L}\left(\lambda,\left\{\mathcal{P}_{i}\right\}\right)$ the irreducible $U_{q}(L \mathfrak{g})$-module corresponding to $\left(\lambda,\left\{\mathcal{P}_{i}\right\}\right) \in \mathcal{P}_{+}^{U}$.

3.4. Composition series. We shall need the analogue for $Y_{\hbar}(\mathfrak{g})$ and $U_{q}(L \mathfrak{g})$ of the existence of composition series in category $\mathcal{O}$ for an arbitrary symmetrizable Kac-Moody algebra $\mathfrak{g}$ (see [36, Lemma 9.6] and [32, Proposition 15]).

\section{Lemma.}

(i) Let $\mathcal{V} \in \mathcal{O}_{\text {int }}\left(U_{q}(L \mathfrak{g})\right)$ and $\lambda \in \mathfrak{h}^{*}$. Then, there exists a filtration of $U_{q}(L \mathfrak{g})$ modules

$$
0=\mathcal{V}_{0} \subset \cdots \subset \mathcal{V}_{t}=\mathcal{V}
$$

such that the following holds for any $\mathcal{V}_{j}, j=1, \ldots, t$ :

- either $\mathcal{V}_{j} / \mathcal{V}_{j-1}=\mathcal{L}\left(\lambda_{j},\left\{\mathcal{P}_{i}^{(j)}\right\}\right)$, for some $\lambda_{j} \geq \lambda$,

- $\operatorname{or}\left(\mathcal{V}_{j} / \mathcal{V}_{j-1}\right)_{\mu}=0$ for every $\mu \geq \lambda$.

Moreover, given $\mu \geq \lambda$ and $\left(\mu,\left\{\mathcal{P}_{i}\right\}\right) \in \mathcal{P}_{+}^{U}$, the number of times $\mathcal{L}\left(\mu,\left\{\mathcal{P}_{i}\right\}\right)$ appears as $\mathcal{V}_{j} / \mathcal{V}_{j-1}$ is independent of $\lambda$ and the filtration chosen, and is denoted by $\left[\mathcal{V}: \mathcal{L}\left(\mu,\left\{\mathcal{P}_{i}\right\}\right)\right]$.

(ii) Let $V \in \mathcal{O}_{\text {int }}\left(Y_{\hbar}(\mathfrak{g})\right)$ and $\lambda \in \mathfrak{h}^{*}$. Then, there exists a filtration of $Y_{\hbar}(\mathfrak{g})$ modules

$$
0=V_{0} \subset \cdots \subset V_{t}=V
$$

such that the following holds for any $V_{j}, j=1, \ldots, t$ :

- either $V_{j} / V_{j-1}=L\left(\lambda_{j},\left\{P_{i}^{(j)}\right\}\right)$, for some $\lambda_{j} \geq \lambda$,

- $\operatorname{or}\left(V_{j} / V_{j-1}\right)_{\mu}=0$ for every $\mu \geq \lambda$.

Moreover, given $\mu \geq \lambda$ and $\left(\mu,\left\{P_{i}\right\}\right) \in \mathcal{P}_{+}^{Y}$, the number of times $L\left(\mu,\left\{P_{i}\right\}\right)$ appears as $V_{j} / V_{j-1}$ is independent of $\lambda$ and the filtration chosen, and is denoted by $\left[V: L\left(\mu,\left\{P_{i}\right\}\right)\right]$.

3.5. Definition of the categories $\mathcal{O}_{\text {int }}^{\Pi}\left(Y_{\hbar}(\mathfrak{g})\right)$ and $\mathcal{O}_{\text {int }}^{\Omega}\left(U_{q}(L \mathfrak{g})\right)$. Let $\Pi \subset \mathbb{C}$ be a subset such that $\Pi \pm \frac{\hbar}{2} \subset \Pi$. We define $\mathcal{O}_{\text {int }}^{\Pi}\left(Y_{\hbar}(\mathfrak{g})\right)$ to be the full subcategory of $\mathcal{O}_{\text {int }}\left(Y_{\hbar}(\mathfrak{g})\right)$ consisting of the representations $V$ such that, for every $\left(\lambda,\left\{P_{i}\right\}\right) \in \mathcal{P}_{+}^{Y}$ for which $\left[V: L\left(\lambda,\left\{P_{i}\right\}\right)\right] \neq 0$, the roots of $P_{i}$ lie in $\Pi$ for every $i \in \mathbf{I}$.

Similarly let $\Omega \subset \mathbb{C}^{\times}$be a subset stable under multiplication by $q^{ \pm 1}$. We define $\mathcal{O}_{\text {int }}^{\Omega}\left(U_{q}(L \mathfrak{g})\right)$ to be the full subcategory of $\mathcal{O}_{\text {int }}\left(U_{q}(L \mathfrak{g})\right)$ consisting of those $\mathcal{V}$ such that for every $\left(\lambda,\left\{\mathcal{P}_{i}\right\}\right) \in \mathcal{P}_{+}^{U}$ for which $\left[\mathcal{V}: \mathcal{L}\left(\lambda,\left\{\mathcal{P}_{i}\right\}\right)\right] \neq 0$, the roots of $\mathcal{P}_{i}$ lie in $\Omega$ for every $i \in \mathbf{I}$. 


\section{Proposition.}

(i) $\mathcal{O}_{\text {int }}^{\Pi}\left(Y_{\hbar}(\mathfrak{g})\right)$ and $\mathcal{O}_{\text {int }}^{\Omega}\left(U_{q}(L \mathfrak{g})\right)$ are Serre subcategories of $\mathcal{O}_{\text {int }}\left(Y_{\hbar}(\mathfrak{g})\right)$ and $\mathcal{O}_{\text {int }}\left(U_{q}(L \mathfrak{g})\right)$, respectively. That is, they are closed under taking direct sums, subobjects, quotients, and extensions.

(ii) When $\mathfrak{g}$ is a simple Lie algebra, $\mathcal{O}_{\text {int }}^{\Pi}\left(Y_{\hbar}(\mathfrak{g})\right)$ and $\mathcal{O}_{\text {int }}^{\Omega}\left(U_{q}(L \mathfrak{g})\right)$ are closed under the tensor product.

Proof. The first part is obvious from the definition. The second part will follow from the alternate characterizations of these categories given in Theorem 3.8 (iv), since by [37, Lemma 1] and [24] $\xi_{i}(u)$ and $\Psi_{i}^{ \pm}(z)$ are grouplike modulo off-diagonal entries.

3.6. Rationality of fields. The aim of this paragraph is to prove that the fields giving the action of the generators of $Y_{\hbar}(\mathfrak{g}), U_{q}(L \mathfrak{g})$ on category $\mathcal{O}$ integrable representations are rational functions. The statement for $U_{q}(L \mathfrak{g})$ is well known; see [5. Section 6] and [33, Proposition 3.8]. Our proof is similar but yields in addition the explicit form of these rational functions.

\section{Proposition.}

(i) Let $V$ be a $Y_{\hbar}(\mathfrak{g})$-module on which $\mathfrak{h}$ acts semisimply with finite-dimensional weight spaces. Then, for every weight $\mu$ of $V$, the generating series

$$
\xi_{i}(u) \in \operatorname{End}\left(V_{\mu}\right)\left[\left[u^{-1}\right]\right], \quad x_{i}^{ \pm}(u) \in \operatorname{Hom}\left(V_{\mu}, V_{\mu \pm \alpha_{i}}\right)\left[\left[u^{-1}\right]\right]
$$

defined in 2.3 are the expansions at $\infty$ of rational functions of $u$. Specifically, let $t_{i, 1}=\xi_{i, 1}-\frac{\hbar}{2} \xi_{i, 0}^{2} \in Y_{\hbar}(\mathfrak{g})^{\mathfrak{h}}$. Then,

$$
x_{i}^{ \pm}(u)=\hbar u^{-1}\left(1 \mp \frac{\operatorname{ad}\left(t_{i, 1}\right)}{2 d_{i} u}\right)^{-1} x_{i, 0}^{ \pm}
$$

and $\xi_{i}(u)=1+\left[x_{i}^{+}(u), x_{i, 0}^{-}\right]$.

(ii) Let $\mathcal{V}$ be a $U_{q}(L \mathfrak{g})$-module on which the operators $K_{h}$ act semisimply with finite-dimensional weight spaces. Then, for every weight $\mu$ of $\mathcal{V}$ and $\varepsilon \in$ $\{ \pm\}$, the generating series

$$
\left.\Psi_{i}(z)^{ \pm} \in \operatorname{End}\left(\mathcal{V}_{\mu}\right)\left[\left[z^{\mp 1}\right]\right], \quad \mathcal{X}_{i}^{\varepsilon}(z)^{ \pm} \in \operatorname{Hom}\left(\mathcal{V}_{\mu}, \mathcal{V}_{\mu \pm \alpha_{i}}\right)\right)\left[\left[z^{\mp 1}\right]\right]
$$

defined in 2.10 are the expansions of rational functions $\Psi_{i}(z), \mathcal{X}_{i}^{\varepsilon}(z)$ at $z=\infty$ and $z=0$. Specifically, let $H_{i, \pm 1}= \pm \Psi_{i, 0}^{\mp} \Psi_{i, \pm 1}^{ \pm} /\left(q_{i}-q_{i}^{-1}\right)$. Then,

$$
\begin{aligned}
& \mathcal{X}_{i}^{\varepsilon}(z)=\left(1-\varepsilon \frac{\operatorname{ad}\left(H_{i, 1}\right)}{[2]_{i} z}\right)^{-1} \mathcal{X}_{i, 0}^{\varepsilon}=-z\left(1-\varepsilon z \frac{\operatorname{ad}\left(H_{i,-1}\right)}{[2]_{i}}\right)^{-1} \mathcal{X}_{i,-1}^{\varepsilon} \\
& \text { and } \Psi_{i}(z)=\Psi_{i, 0}^{-}+\left(q_{i}-q_{i}^{-1}\right)\left[\mathcal{X}_{i}^{+}(z), \mathcal{X}_{i, 0}^{-}\right] .
\end{aligned}
$$

Proof. (i) The relations (Y2) and (Y3) imply that $\left[t_{i, 1}, x_{i, r}^{ \pm}\right]= \pm 2 d_{i} x_{i, r+1}^{ \pm}$, so that

$$
\left[t_{i, 1}, x_{i}^{ \pm}(u)\right]= \pm 2 d_{i}\left(u x_{i}^{ \pm}(u)-\hbar x_{i, 0}\right) .
$$

The relation $\xi_{i}(u)=1+\left[x_{i}^{+}(u), x_{i, 0}^{-}\right]$is a direct consequence of (Y5). 
(ii) The relations (QL2) and (QL3) imply that $\left[H_{i, \pm 1}, \mathcal{X}_{i, k}^{\varepsilon}\right]=\varepsilon[2]_{i} \mathcal{X}_{i, k \pm 1}^{\varepsilon}$, and therefore that

$$
\begin{aligned}
& \mathcal{X}_{i}^{\varepsilon}(z)^{+}=\left(1-\varepsilon \frac{\operatorname{ad}\left(H_{i, 1}\right)}{[2]_{i} z}\right)^{-1} \mathcal{X}_{i, 0}^{\varepsilon}, \\
& \mathcal{X}_{i}^{\varepsilon}(z)^{-}=-z\left(1-\varepsilon z \frac{\operatorname{ad}\left(H_{i,-1}\right)}{[2]_{i}}\right)^{-1} \mathcal{X}_{i,-1}^{\varepsilon},
\end{aligned}
$$

which are rational functions regular at $z=\infty$ and $z=0$, respectively. To see that these functions are the same, it suffices to note that, as a formal power series, and therefore as a rational function, $\mathcal{X}_{i}^{\varepsilon}(z)^{-}$also satisfies

$$
\left(1-\varepsilon \frac{\operatorname{ad}\left(H_{i, 1}\right)}{[2]_{i} z}\right) \mathcal{X}_{i}^{\varepsilon}(z)^{-}=\mathcal{X}_{i, 0}^{\varepsilon} .
$$

Finally, note that $\Psi_{i}^{ \pm}(z)=\Psi_{i, 0}^{-}+\left(q_{i}-q_{i}^{-1}\right)\left[\mathcal{X}_{i}^{+}(z)^{ \pm}, \mathcal{X}_{i, 0}^{-}\right]$.

3.7. The following result will be needed elsewhere.

\section{Lemma.}

(i) Let $V \in \mathcal{O}_{\text {int }}\left(Y_{\hbar}(\mathfrak{g})\right)$, and let $\mu \in \mathfrak{h}^{*}$ be a weight of $V$. Then, the poles of $\xi_{i}(u)_{\mu}$ are contained in the union of the poles of $x_{i}^{ \pm}(u)_{\mu}$ and those of $x_{i}^{ \pm}(u)_{\mu \mp \alpha_{i}}$.

(ii) Let $\mathcal{V} \in \mathcal{O}_{\text {int }}\left(U_{q}(L \mathfrak{g})\right)$, and let $\mu \in \mathfrak{h}^{*}$ be a weight of $\mathcal{V}$. Then the poles of $\Psi_{i}(z)_{\mu}$ are contained in the union of the poles of $\mathcal{X}_{i}^{ \pm}(z)_{\mu}$ and those of $\mathcal{X}_{i}^{ \pm}(z)_{\mu \mp \alpha_{i}}$.

Proof. (i) and (ii) follow from

$$
\begin{aligned}
\xi_{i}(u)=1+\left[x_{i}^{+}(u), x_{i, 0}^{-}\right] & =1+\left[x_{i, 0}^{+}, x_{i}^{-}(u)\right], \\
\Psi_{i}(z)=\Psi_{i, 0}^{-}+\left(q_{i}-q_{i}^{-1}\right)\left[\mathcal{X}_{i}^{+}(z), \mathcal{X}_{i, 0}^{-}\right] & =\Psi_{i, 0}^{-}+\left(q_{i}-q_{i}^{-1}\right)\left[\mathcal{X}_{i, 0}^{+}, \mathcal{X}_{i}^{-}(z)\right],
\end{aligned}
$$

respectively.

3.8. Equivalent characterizations of $\mathcal{O}_{\text {int }}^{\Pi}\left(Y_{\hbar}(\mathfrak{g})\right)$ and $\mathcal{O}_{\text {int }}^{\Omega}\left(U_{q}(L \mathfrak{g})\right)$. In view of Proposition [3.6. we can define, for a given representation $(\rho, V)$ in $\mathcal{O}_{\text {int }}\left(Y_{\hbar}(\mathfrak{g})\right)$, a subset $\sigma(V) \subset \mathbb{C}$ consisting of the poles of the rational functions $\left\{\rho\left(\xi_{i}(u)\right)^{ \pm 1}\right.$, $\left.\rho\left(x_{i}^{ \pm}(u)\right)\right\}$. Similarly, for a representation $(\rho, \mathcal{V})$ in $\mathcal{O}_{\text {int }}\left(U_{q}(L \mathfrak{g})\right)$, we define a subset $\sigma(\mathcal{V}) \subset \mathbb{C}^{\times}$consisting of poles of the functions $\left\{\rho\left(\Psi_{i}(z)\right)^{ \pm 1}, \rho\left(\mathcal{X}_{i}^{ \pm}(z)\right)\right\}$.

\section{Theorem.}

(1) Let $V \in \mathcal{O}_{\text {int }}\left(Y_{\hbar}(\mathfrak{g})\right)$. Then, the following conditions are equivalent:

(i) $V \in \mathcal{O}_{\text {int }}^{\Pi}\left(Y_{\hbar}(\mathfrak{g})\right)$.

(ii) $\sigma(V) \subset \Pi$.

(iii) The poles of $\xi_{i}(u)^{ \pm 1}$ are contained in $\Pi$.

(iv) The eigenvalues of $\xi_{i}(u)$ have zeros and poles in $\Pi$.

(2) Let $\mathcal{V} \in \mathcal{O}_{\text {int }}\left(U_{q}(L \mathfrak{g})\right)$. Then, the following conditions are equivalent:

(i) $\mathcal{V} \in \mathcal{O}_{\text {int }}^{\Omega}\left(U_{q}(L \mathfrak{g})\right)$

(ii) $\sigma(\mathcal{V}) \subset \Omega$.

(iii) The poles of $\Psi_{i}(z)^{ \pm 1}$ are contained in $\Omega$.

(iv) The eigenvalues of $\Psi_{i}(z)$ have zeros and poles in $\Omega$. 
Proof. The rest of this section is devoted to the proof of Theorem 3.8 for the Yangian $Y_{\hbar}(\mathfrak{g})$. The assertion for $U_{q}(L \mathfrak{g})$ is proved similarly, and we therefore omit it. The following implications are obvious: (ii) $\Rightarrow$ (iii) $\Rightarrow$ (iv) $\Rightarrow$ (i). We prove (i) $\Rightarrow$ (ii) by induction on the length of the composition series of $V$. More precisely, assume (i), and fix a weight $\mu \in \mathfrak{h}^{*}$. We need to prove that the operators $\left\{\xi_{i}(u)_{\mu}^{ \pm 1}, x_{i}^{ \pm}(u)_{\mu}\right\}_{i \in \mathbf{I}}$ have poles in $\Pi$. Fix an arbitrary $\lambda \in \mathfrak{h}^{*}$ such that $\lambda \leq \mu-\alpha_{i}$ for every $i \in \mathbf{I}$, and consider a composition series given by Lemma 3.4. The proof of (i) $\Rightarrow$ (ii) is by induction on the length of this composition series. Proposition 3.9 deals with the case of an irreducible $V$, while Proposition 3.10 carries out the induction step.

3.9 .

Proposition. Let $V$ be an irreducible representation in $\mathcal{O}_{\text {int }}\left(Y_{\hbar}(\mathfrak{g})\right)$. Then $V \in$ $\mathcal{O}_{\text {int }}^{\Pi}\left(Y_{\hbar}(\mathfrak{g})\right)$ if and only if $\sigma(V) \subset \Pi$.

Proof. It is clear that if $\sigma(V) \subset \Pi$, the roots of $P_{i}(u)$ are in $\Pi$ (since roots of $P_{i}(u)$ are obtained by successively shifting the poles of $\xi_{i}(u)$ acting on the highest weight vector by $d_{i} \hbar$, and $\Pi$ is closed under these shifts). Hence, by definition, $V \in \mathcal{O}_{\text {int }}^{\Pi}\left(Y_{\hbar}(\mathfrak{g})\right)$.

Assuming now that the roots of $P_{i}(u)$ are in $\Pi$, for every $i \in \mathbf{I}$, we shall prove that all the rational functions $\left\{\xi_{i}(u), x_{i}^{ \pm}(u)\right\}$ have poles in $\Pi$. Let

$$
V=\bigoplus_{\nu \in \mathfrak{h}^{*}} V_{\nu}
$$

be the weight space decomposition of $V$ as a $\mathfrak{g}$-module and, for $y \in Y_{\hbar}(\mathfrak{g})$, write $y_{\nu}$ for the restriction of $y$ to $V_{\nu}$.

Let $\mu \in \mathfrak{h}^{*}$ be the highest weight of $V$. Using the results of 3.2 we have the following properties of $V$, which will be used in the proof:

(P1) Every weight space $V_{\nu}$, for $\nu<\mu$, is spanned by $\left\{x_{i, r}^{-} V_{\nu+\alpha_{i}}\right\}_{i \in \mathbf{I}, r \in \mathbb{N}}$.

(P2) If $v \in V_{\nu}$ is annihilated by $x_{i, r}^{+}$for every $i \in \mathbf{I}$ and $r \in \mathbb{N}$, and $\nu \neq \mu$, then $v=0$.

The proof that each rational function $\xi_{i}(u)_{\nu}, x_{i}^{ \pm}(u)_{\nu}$ has poles in $\Pi$ is by induction on the height of $\mu-\nu$. Recall that for an element $\alpha=\sum_{i} n_{i} \alpha_{i} \in Q_{+}$, the height of $\alpha$, denoted by ht $(\alpha)$, is defined as

$$
\operatorname{ht}(\alpha)=\sum_{i} n_{i} \in \mathbb{N} .
$$

More precisely, we prove the following statement by induction on $k \in \mathbb{N}$ :

$\mathbf{S}(k)$ : For every $i \in \mathbf{I}$, the rational functions $\xi_{i}(u)_{\nu}, x_{i}^{+}(u)_{\nu}$ have poles in $\Pi$, for every $\nu$ such that $\operatorname{ht}(\mu-\nu) \leq k$; and $x_{i}^{-}(u)_{\nu}$ has poles in $\Pi$ for every $\nu$ such that ht $(\mu-\nu)<k$.

The base case $\mathbf{S}(0)$ is clear since $x_{i}^{+}(u)_{\mu}=0$ and

$$
\xi_{i}(u)_{\mu}=\frac{P_{i}\left(u+d_{i} \hbar\right)}{P_{i}(u)} .
$$

Let us assume that $\mathbf{S}\left(k^{\prime}\right)$ holds for every $k^{\prime} \leq k$, where $k \geq 0$, and prove $\mathbf{S}(k+1)$. Let $\nu$ be a weight of $V$ such that $h t(\mu-\nu)=k+1$, and let $i, j \in \mathbf{I}$. Using the relation $(\mathcal{Y} 5)$ we have

$$
x_{i}^{+}(u)_{\nu} x_{j}^{-}(v)_{\nu+\alpha_{j}}=x_{j}^{-}(v)_{\nu+\alpha_{i}+\alpha_{j}} x_{i}^{+}(u)_{\nu+\alpha_{j}}+\frac{\delta_{i j} \hbar}{u-v}\left(\xi_{i}(v)_{\nu+\alpha_{i}}-\xi_{i}(u)_{\nu+\alpha_{i}}\right) .
$$


Note that the right-hand side has poles in $\Pi \times \Pi$ by the induction hypothesis. This allows us to conclude the same for $x_{i}^{+}(u)_{\nu}$ and $x_{j}^{-}(v)_{\nu+\alpha_{j}}$ as follows. Assume that $x_{j}^{-}(v)_{\nu+\alpha_{j}}$ has a pole at $z \in \mathbb{C} \backslash \Pi$ of order $n$. Multiplying both sides of Equation (3.1) by $(v-z)^{n}$ and setting $v=z$, we get

$$
x_{i}^{+}(u)_{\nu}\left(\left.(v-z)^{n} x_{j}^{-}(v)_{\nu+\alpha_{j}}\right|_{v=z}\right)=0 .
$$

Thus the image of the operator $\left(\left.(v-z)^{n} x_{j}^{-}(v)_{\nu+\alpha_{j}}\right|_{v=z}\right)$ is annihilated by all $x_{i, r}^{+}$, which implies that this operator is zero, since $\nu \neq \mu$ (see property (P2) above). This is a contradiction to the fact that $z$ is a pole of order $n$ of $x_{j}^{-}(v)_{\nu+\alpha_{j}}$. The proof for $x_{i}^{+}(u)_{\nu}$ is similar.

It remains to show that $\xi_{i}(u)_{\nu}$ has poles in $\Pi$. For this we use the relation $(\mathcal{Y} 3)$ $\xi_{i}(u)_{\nu} x_{j}^{-}(v)_{\nu+\alpha_{j}}=\frac{u-v-a}{u-v+a} x_{j}^{-}(v)_{\nu+\alpha_{j}} \xi_{i}(u)_{\nu+\alpha_{j}}+\frac{2 a}{u-v+a} x_{j}^{-}(u+a)_{\nu+\alpha_{j}} \xi_{i}(u)_{\nu+\alpha_{j}}$, where $a=\hbar d_{i} a_{i j} / 2$.

This relation implies that the poles of $\xi_{i}(u)$ acting on the image of the operator $x_{j}^{-}(v)_{\nu+\alpha_{j}}$ in $V_{\nu}$ are the poles of either $\xi_{i}(u)_{\nu+\alpha_{j}}$ or $x_{j}^{-}(u+a)_{\nu+\alpha_{j}}$, which are in $\Pi$, by the induction hypothesis and the fact that $\Pi$ is stable under the shift by $a$. Since $V_{\nu}$ is spanned by such subspaces (property $(\mathrm{P} 1)$ ), we are done.

3.10 .

Proposition. Let $0 \rightarrow V_{1} \rightarrow V \rightarrow V_{2} \rightarrow 0$ be a short exact sequence of integrable $Y_{\hbar}(\mathfrak{g})$-modules. If $\sigma\left(V_{1}\right), \sigma\left(V_{2}\right) \subset \Pi$, then $\sigma(V) \subset \Pi$.

Proof. Let us fix $\mu \in \mathfrak{h}^{*}$ and $i \in \mathbf{I}$. We will prove that for every $k \in \mathbb{Z}$ the poles of $\xi_{i}(u)_{\mu+k \alpha_{i}}, x_{i}^{ \pm}(u)_{\mu+k \alpha_{i}}$ acting on the corresponding weight space of $V$ are in $\Pi$.

We write $V=V_{1} \oplus V_{2}$ as vector spaces. An element $y \in Y_{\hbar}(\mathfrak{g})$ has the following form (viewed as an element of $\operatorname{End}(V)$ ):

$$
y=\left[\begin{array}{cc}
y^{11} & y^{12} \\
0 & y^{22}
\end{array}\right] .
$$

By assumption, $\xi_{i}(u)^{l l}, x_{i}^{ \pm}(u)^{l l}$ have poles in $\Pi$, for $l=1,2$. Let us assume that $z \notin \Pi$ is a pole of one of the functions $\xi_{i}(u)_{\nu}^{12}, x_{i}^{ \pm}(u)_{\nu}^{12}$ for $\nu \in \mu+\mathbb{Z} \alpha_{i}$. Let $N$ be the maximum of the order of the pole at $z$ of these functions (note that by definition, there are only finitely many $k \in \mathbb{Z}$ such that $V_{\mu+k \alpha_{i}} \neq 0$ ). Define for every $\nu \in \mu+\mathbb{Z} \alpha_{i}$

$$
\begin{aligned}
\mathrm{H}_{\nu} & =\lim _{u \rightarrow z}(u-z)^{N} \xi_{i}(u)_{\nu}^{12}, \\
\mathrm{X}_{\nu}^{ \pm} & =\lim _{u \rightarrow z}(u-z)^{N} x_{i}^{ \pm}(u)_{\nu}^{12} .
\end{aligned}
$$

Using the relation $(\mathcal{Y} 5)$ and taking its $(1,2)$ entry, we have

$$
\begin{aligned}
& (u-v)\left(x_{i}^{+}(u)_{\nu-\alpha_{i}}^{11} x_{i}^{-}(v)_{\nu}^{12}+x_{i}^{+}(u)_{\nu-\alpha_{i}}^{12} x_{i}^{-}(v)_{\nu}^{22}\right. \\
& \left.\quad-x_{i}^{-}(v)_{\nu+\alpha_{i}}^{11} x_{i}^{+}(u)_{\nu}^{12}-x_{i}^{-}(v)_{\nu+\alpha_{i}}^{12} x_{i}^{+}(u)_{\nu}^{22}\right)=\hbar\left(\xi_{i}(v)_{\nu}^{12}-\xi_{i}(u)_{\nu}^{12}\right) .
\end{aligned}
$$

Multiplying both sides by $(u-z)^{N}$ and letting $u \rightarrow z$ we get

$$
(z-v)\left(\mathbf{X}_{\nu-\alpha_{i}}^{+} x_{i}^{-}(v)_{\nu}^{22}-x_{i}^{-}(v)_{\nu+\alpha_{i}}^{11} \mathbf{X}_{\nu}^{+}\right)=-\hbar \mathrm{H}_{\nu},
$$


and similarly (using $v-z$ instead of $u-z$ ) we have

$$
(u-z)\left(x_{i}^{+}(u)_{\nu-\alpha_{i}}^{11} \mathrm{X}_{\nu}^{-}-\mathrm{X}_{\nu+\alpha_{i}}^{-} x_{i}^{+}(u)_{\nu}^{22}\right)=\hbar \mathrm{H}_{\nu} .
$$

These equations clearly imply that $\mathrm{H}_{\nu}=0$ and we have the following relations:

$$
\begin{aligned}
& \mathrm{X}_{\nu-\alpha_{i}}^{+} x_{i}^{-}(v)_{\nu}^{22}=x_{i}^{-}(v)_{\nu+\alpha_{i}}^{11} \mathrm{X}_{\nu}^{+}, \\
& x_{i}^{+}(u)_{\nu-\alpha_{i}}^{11} \mathrm{X}_{\nu}^{-}=\mathrm{X}_{\nu+\alpha_{i}}^{-} x_{i}^{+}(u)_{\nu}^{22} .
\end{aligned}
$$

Now we consider the relation $(\mathcal{Y} 3)$ in the following form:

$$
\begin{aligned}
\left(u-v-d_{i} \hbar\right) \xi_{i}(u) x_{i}^{+}(v)-\left(u-v+d_{i} \hbar\right) x_{i}^{+} & (v) \xi_{i}(u) \\
& =-d_{i} \hbar\left(\xi_{i}(u) x_{i}^{+}(u)+x_{i}^{+}(u) \xi_{i}(u)\right) .
\end{aligned}
$$

Again taking its $(1,2)$ component, multiplying with $(u-z)^{N}$ and letting $u \rightarrow z$, and using the fact that $\mathrm{H}=0$ we get

$$
\xi_{i}(z)_{\nu+\alpha_{i}}^{11} \mathrm{X}_{\nu}^{+}+\mathrm{X}_{\nu}^{+} \xi_{i}(z)_{\nu}^{22}=0
$$

Similar computation with relation $(\mathcal{Y} 4)$ yields

$$
\begin{aligned}
\left(z-v-d_{i} \hbar\right) \mathrm{X}_{\nu+\alpha_{i}}^{+} x_{i}^{+}(v)_{\nu}^{22}-(z- & \left.+d_{i} \hbar\right) x_{i}^{+}(v)_{\nu+\alpha_{i}}^{11} \mathrm{X}_{\nu}^{+} \\
& =-d_{i} \hbar\left(x_{i}^{+}(z)_{\nu+\alpha_{i}}^{11} \mathrm{X}_{\nu}^{+}+\mathrm{X}_{\nu+\alpha_{i}}^{+} x_{i}^{+}(z)_{\nu}^{22}\right) .
\end{aligned}
$$

We take the commutator of (3.5) with $x_{i, 0}^{-}$, using the commutativity property (3.2), Equation (3.4), and the relation $\left[x_{i}^{+}(u), x_{i, 0}^{-}\right]=\xi_{i}(u)-1$, to obtain

$$
\left(z-v-d_{i} \hbar\right) \mathbf{X}_{\nu}^{+} \xi_{i}(v)_{\nu}^{22}-\left(z-v+d_{i} \hbar\right) \xi_{i}(v)_{\nu+\alpha_{i}}^{11} \mathbf{X}_{\nu}^{+}=0 .
$$

If we set $v=z-d_{i} \hbar$ in this equation, we get

$$
-2 d_{i} \hbar \xi_{i}\left(z-d_{i} \hbar\right)_{\nu+\alpha_{i}}^{11} \mathrm{X}_{\nu}^{+}=0 .
$$

But since $z-d_{i} \hbar \notin \Pi$ and $\xi_{i}(u)^{11}$ is invertible there, we obtain that $\mathrm{X}_{\nu}^{+}=0$. A similar argument shows that $\mathrm{X}_{\nu}^{-}=0$, which contradicts the fact that $N$ was the order of the pole at $z$ of one of $\xi_{i}(u)_{\nu}^{12}, x_{i}^{ \pm}(u)_{\nu}^{12}$. This contradiction proves that all the poles are in $\Pi$ and we are done.

\section{Additive difference equations}

In this section, we give a self-contained account of Birkhoff's theory of additive difference equations, including a detailed discussion of the inverse problem for abelian ones. Our exposition and understanding of the subject owe much to [6, 8, 38. Additional information may be found in [7, 50].

4.1. Let $V$ be a finite-dimensional vector space over $\mathbb{C}$, and let $A: \mathbb{C} \rightarrow \operatorname{End}(V)$ be a rational function. We assume that $A$ is regular at $\infty$ and such that $A(\infty)=$ 1. Consider the following system of difference equations for a function $\phi: \mathbb{C} \rightarrow$ $\operatorname{End}(V)$ :

$$
\phi(u+1)=A(u) \phi(u) .
$$

Let $\mathcal{A} \subseteq \operatorname{End}(V)$ be the subalgebra generated by $A(u), u \in \mathbb{C}$, or equivalently by the coefficients of the Taylor expansion

$$
A(u)=1+A_{0} u^{-1}+A_{1} u^{-2}+\cdots
$$


of $A$ near $\infty$. The system (4.1) is said to be non-resonant relative to a subalgebra $\widetilde{\mathcal{A}} \subseteq \operatorname{End}(V)$ containing $\mathcal{A}$ if the eigenvalues of $\operatorname{ad}\left(A_{0}\right)$ on $\widetilde{\mathcal{A}}$ do not lie in $\mathbb{Z}_{\neq 0}$. Note that this is the case in either of the following situations:

- The eigenvalues of $A_{0}$ do not differ by positive integers, and $\widetilde{\mathcal{A}}=\operatorname{End}(V)$.

- The system (4.1) is abelian, that is, $[A(u), A(v)]=0$ for any $u, v \in \mathbb{C}$, and $\widetilde{\mathcal{A}}=\mathcal{A}$.

If (4.1) is non-resonant relative to $\widetilde{\mathcal{A}}$, it is easy to see that it possesses a unique formal solution of the form $\Upsilon(u) u^{A_{0}}$, where 5

$$
\Upsilon(u)=1+\Upsilon_{0} u^{-1}+\Upsilon_{1} u^{-2}+\cdots \in \widetilde{\mathcal{A}}\left[\left[u^{-1}\right]\right] .
$$

\subsection{Canonical fundamental solutions.}

Theorem ([6]). If the system (4.1) is non-resonant relative to $\widetilde{\mathcal{A}} \subseteq \operatorname{End}(V)$, there exist unique meromorphic solutions $\phi^{ \pm}: \mathbb{C} \rightarrow \widetilde{\mathcal{A}}$ such that

(i) $\phi^{ \pm}$is holomorphic and invertible for $\pm \operatorname{Re}(u) \gg 0$.

(ii) $\phi^{ \pm}$possesses an asymptotic expansion of the form

$$
\phi^{ \pm}(u) \sim\left(1+H_{0}^{ \pm} u^{-1}+H_{1}^{ \pm} u^{-2}+\cdots\right) \cdot( \pm u)^{A_{0}}
$$

in any right (resp. left) half-plane, where $v^{A_{0}}=\exp \left(A_{0} \log (v)\right)$ is defined by taking the standard determination of the logarithm on $\mathbb{C} \backslash \mathbb{R}_{\leq 0}$.

Moreover,

(iii) The poles of $\left(\phi^{-}\right)^{ \pm 1}$ are contained in $\mathcal{P}+\mathbb{Z}_{>0}$ and $\mathcal{Z}+\mathbb{Z}_{>0}$, respectively, where $\mathcal{P}, \mathcal{Z}$ are the poles of $A(u), A(u)^{-1}$.

(iv) The poles of $\left(\phi^{+}\right)^{ \pm 1}$ are contained in $\mathcal{Z}-\mathbb{N}$ and $\mathcal{P}-\mathbb{N}$, respectively.

(v) The asymptotics (4.3) of $\phi^{ \pm}$are given by $\Upsilon(u)( \pm u)^{A_{0}}$.

Proof. The uniqueness of $\phi^{ \pm}$is proved in 4.3. In 4.4 4.6, we give a proof of the existence of the fundamental solutions $\phi^{ \pm}$under the simplifying assumption that $A_{0}$ commutes with $A(u)$ for every $u$. The general case is treated in 6,38 .

(iii) and (iv) If $\phi^{-}(u)$ is holomorphic and invertible for $\operatorname{Re}(u)<a$, then for $\operatorname{Re}(u)<a+n, \phi^{-}(u)=A(u-1) \cdots A(u-n) \phi^{-}(u-n)\left(\operatorname{resp} . \quad\left(\phi^{-}(u)\right)^{-1}=\right.$ $\left.\phi^{-}(u-n)^{-1} A(u-n)^{-1} \cdots A(u-1)^{-1}\right)$, which has poles only if one of $u-1, \ldots, u-n$ is a pole of $A(u)$ (resp. of $\left.A(u)^{-1}\right)$. This is similar for $\phi^{+}$.

(v) Let $H^{ \pm}(u) \in 1+u^{-1} \widetilde{\mathcal{A}}\left[\left[u^{-1}\right]\right]$ be such that $\phi^{ \pm}( \pm u)^{-A_{0}} \sim H^{ \pm}(u)$ for $\pm \operatorname{Re}(u)>a$. Multiplying both sides of $\phi^{ \pm}(u+1)=A(u) \phi^{ \pm}(u)$ by $( \pm(u+1))^{-A_{0}}$, using the fact that $( \pm u)^{A_{0}}( \pm(u+1))^{-A_{0}}=(1+1 / u)^{-A_{0}}$ for $u \notin \mathbb{R}_{\leq 0}$ (resp. $u \notin$ $\left.\mathbb{R}_{>-1}\right)$, and taking asymptotic expansions show that $H^{ \pm}(u+1)=A(u) H^{ \pm}(u)(1+$ $1 / u)^{-A_{0}}$, so that $H^{ \pm}(u)=\Upsilon(u)$ by uniqueness.

4.3. Uniqueness of fundamental solutions. Let $\phi_{1}^{+}(u), \phi_{2}^{+}(u)$ be two solutions of (4.1) satisfying the conditions (i) and (ii) of Theorem 4.2, and set $C(u)=$ $\left(\phi_{1}^{+}(u)\right)^{-1} \phi_{2}^{+}(u) . C(u)$ is 1-periodic and holomorphic for $\operatorname{Re}(u) \gg 0$ and therefore on the entire complex plane. As a holomorphic function of $z=e^{2 \pi \iota u}=$ $e^{2 \pi \iota \operatorname{Re}(u)} e^{-2 \pi \operatorname{Im}(u)}, C$ has removable singularities at $z=0$ and $z=\infty$. Indeed,

$$
C(u)=u^{-A_{0}}\left(\phi_{1}^{+}(u) u^{-A_{0}}\right)^{-1}\left(\phi_{2}^{+}(u) u^{-A_{0}}\right) u^{A_{0}} .
$$

\footnotetext{
${ }^{5}$ For the formal solution $\Upsilon(u) u^{A_{0}}$, Equation 4.1 is understood to mean $\Upsilon(u+1)=$ $A(u) \Upsilon(u)(1+1 / u)^{-A_{0}}$, where $(1+1 / u)^{-A_{0}}=\sum_{r \geq 0}(-1)^{r} \frac{A_{0}\left(A_{0}+1\right) \cdots\left(A_{0}+r-1\right)}{r !} u^{-r}$.
} 
Since $C$ is 1-periodic, we may assume that $\operatorname{Re} u \gg 0$, so that the second and third factors tend to 1 as $\operatorname{Im} u \rightarrow \pm \infty$. Since the first and last ones grow like a polynomial in $u$, it follows that $\lim _{z \rightarrow 0} z C(z)=0=\lim _{z \rightarrow \infty} z^{-1} C(z)$. Thus, $C(u) \equiv C$ is a constant element of $\widetilde{\mathcal{A}}$. By (4.4),

$$
u^{A_{0}} C u^{-A_{0}} \sim 1+C^{0} u^{-1}+\cdots
$$

for $\operatorname{Re} u \gg 0$. Let $A_{0}=S_{0}+N_{0}$ be the Jordan decomposition of $A_{0}$ and $C=$ $\sum_{\lambda \in \mathbb{C}} C_{\lambda}$ the decomposition of $C$ into eigenvectors of ad $\left(S_{0}\right)$. Since

$$
\operatorname{Ad}\left(u^{A_{0}}\right) C_{\lambda}=u^{\lambda} \sum_{k \geq 0} \log (u)^{k} \frac{\operatorname{ad}\left(N_{0}\right)^{k}}{k !} C_{\lambda},
$$

it follows that each $C_{\lambda}$ is an eigenvector of $\operatorname{ad}\left(A_{0}\right)$, that $C_{\lambda}=0$ for any $\lambda \notin \mathbb{Z}_{\leq 0}$, and that $C_{0}=1$. The non-resonance condition then implies that $C_{n}=0$ for any $n \in \mathbb{Z}_{<0}$, so that $C=1$. The uniqueness of $\phi^{-}$follows in the same way.

4.4. Existence of fundamental solutions, I. Assume first that $A_{0}=0$. The system (4.1) is then called regular, and its fundamental solutions are given by

$$
\begin{aligned}
\phi^{+}(u) & =\prod_{n \geq 0}^{\rightarrow} A(u+n)^{-1}=A(u)^{-1} A(u+1)^{-1} \cdots, \\
\phi^{-}(u) & =\prod_{n \geq 1}^{\rightarrow} A(u-n)=A(u-1) A(u-2) \cdots .
\end{aligned}
$$

It is easy to prove that the above products converge locally uniformly on the complement of $\mathcal{Z}-\mathbb{N}, \mathcal{P}+\mathbb{Z}_{>0}$, respectively, and have the required asymptotics.

4.5. Holomorphic functional calculus [17]. Recall that given $X \in \operatorname{End}(V)$, and a meromorphic function $f$ which is holomorphic on a neighborhood of the eigenvalues of $X, f(X) \in \operatorname{End}(V)$ is defined by the Cauchy integral

$$
f(X)=\frac{1}{2 \pi \iota} \int_{C} f(v)(v-X)^{-1} d v,
$$

where $C$ is any contour enclosing all of the eigenvalues of $X$ and none of the poles of $f(v)$. If $X$ is semisimple, with eigenvalues $\lambda_{1}, \ldots, \lambda_{k}$ and corresponding eigenspaces $V_{1}, \ldots, V_{k}$, the residue theorem shows that $f(X)$ is the semisimple endomorphism acting as multiplication by $f\left(\lambda_{i}\right)$ on $V_{i}$. More generally, if $X=X_{S}+X_{N}$ is the Jordan decomposition of $X$, expanding $(v-X)^{-1}$ as

$$
(v-X)^{-1}=\sum_{k \geq 0}\left(v-X_{S}\right)^{-k-1} X_{N}^{k},
$$

a finite sum since $X_{N}$ is nilpotent, shows that

$$
f(X)=\sum_{k \geq 0} \frac{f^{(k)}\left(X_{S}\right)}{k !} X_{N}^{k} .
$$

If $f(u)=\sum_{i=0}^{n} a_{i} u^{i}$ is a polynomial, then $f(X)=\sum_{i} a_{i} X^{i}$. Moreover, $f_{1}$. $f_{2}(X)=f_{1}(X) f_{2}(X)$ when both sides are defined. Finally, if $f_{n}$ is a sequence 
converging locally uniformly to $f$, then $f_{n}(X)$ converges to $f(X)$. It follows for example that if

$$
\Gamma(v)=v^{-1} e^{-\gamma v} \prod_{n \geq 1}\left(1+\frac{v}{n}\right)^{-1} e^{v / n}
$$

is the Euler Gamma function, where $\gamma$ is the Euler-Mascheroni constant, then

$$
\Gamma(X)=X^{-1} e^{-\gamma X} \prod_{n \geq 1}\left(1+\frac{X}{n}\right)^{-1} e^{X / n} .
$$

4.6. Existence of fundamental solutions, II. Assume now that $A_{0}$ commutes with $A(u)$. We may then regularize (4.1) as follows. Set

$$
\bar{A}(u)=\left(1-A_{0} u^{-1}\right) A(u) .
$$

The difference equation $\bar{\phi}(u+1)=\bar{A}(u) \bar{\phi}(u)$ is regular, and its fundamental solutions $\bar{\phi}^{ \pm}$can be constructed as in 4.4. The fundamental solutions to (4.1) are $\phi^{ \pm}(u)=f^{ \pm}(u) \bar{\phi}^{ \pm}(u)$, where $f^{ \pm}(u)$ are the solutions of $f(u+1)=u\left(u-A_{0}\right)^{-1} f(u)$ given by

$$
\begin{aligned}
& f^{+}(u)=\Gamma(u) \Gamma\left(u-A_{0}\right)^{-1}, \\
& f^{-}(u)=\Gamma\left(1-u+A_{0}\right) \Gamma(1-u)^{-1} .
\end{aligned}
$$

We will show in 4.7 that $f^{ \pm}(u)$ have the required asymptotics as $u \rightarrow \infty$. Assuming this for now, and using (4.6), we can rewrite $\phi^{ \pm}$as

$$
\begin{aligned}
& \phi^{+}(u)=e^{-\gamma A_{0}} A(u)^{-1} \prod_{n \geq 1}^{\rightarrow} A(u+n)^{-1} e^{A_{0} / n}, \\
& \phi^{-}(u)=e^{-\gamma A_{0}} \prod_{n \geq 1}^{\rightarrow} A(u-n) e^{A_{0} / n} .
\end{aligned}
$$

4.7. Asymptotics of $f^{ \pm}$. Recall Sterling's asymptotic expansion [52, Section 12.33]

$$
\Gamma(u) \sim e^{-u} u^{u-1 / 2} \sqrt{2 \pi}\left(1+1 / 12 u+1 / 288 u^{2}+\cdots\right),
$$

valid on $\mathbb{C} \backslash \mathbb{R}_{\leq 0}$. This implies that, for any $\lambda \in \mathbb{C}$,

$$
\frac{\Gamma(u-\lambda)}{\Gamma(u)} \sim u^{-\lambda}\left(1+h_{1} u^{-1}+\cdots\right)
$$

and, differentiating with respect to $\lambda$, that, for any $k \geq 0$,

$$
\frac{\Gamma^{(k)}(u)}{\Gamma(u)} \sim(\log (u))^{k}\left(1+h_{1}^{(k)} u^{-1}+\cdots\right) .
$$

Let now $A_{0}=S_{0}+N_{0}$ be the Jordan decomposition of $A_{0}$. By (4.5),

$$
\begin{aligned}
f^{+}(u)^{-1} & =\Gamma\left(u-A_{0}\right) \Gamma(u)^{-1} \\
& =\frac{\Gamma\left(u-S_{0}\right)}{\Gamma(u)} \sum_{k \geq 0} \frac{\Gamma^{(k)}\left(u-S_{0}\right)}{\Gamma\left(u-S_{0}\right)} \frac{\left(-N_{0}\right)^{k}}{k !} \\
& \sim u^{-A_{0}}(1+\cdots) .
\end{aligned}
$$

Similarly, $f^{-}(u) \sim(1+\cdots)(-u)^{A_{0}}$. 
4.8. Connection matrix. Define the connection matrix of the system (4.1) to be the function $S: \mathbb{C} \rightarrow G L(V)$ given by

$$
S(u)=\left(\phi^{+}(u)\right)^{-1} \phi^{-}(u) .
$$

Clearly, $S(u)$ is 1-periodic, and therefore a function of $z=e^{2 \pi \iota u}$.

Proposition. $S$ is a rational function of $z$, regular at $0, \infty$, and such that

$$
S(\infty)=e^{\pi \iota A_{0}} \quad \text { and } \quad S(0)=e^{-\pi \iota A_{0}} .
$$

Proof. By Theorem 4.2, (iii) and (iv), $S$ is a meromorphic function with finitely many poles in the $z$-plane. To determine its behavior as $z \rightarrow \infty$ (resp. $z \rightarrow 0$ ), it suffices to consider the limit of $S(u)$ as $\operatorname{Im}(u) \rightarrow \mp \infty$, with Re $u$ lying in an interval of the form $\left[a, a+1\left[\right.\right.$. Since both asymptotic expansions $\phi^{ \pm} \sim \Upsilon(u)( \pm u)^{A_{0}}$ hold on the strip $\operatorname{Re}(u) \in[a, a+1[$, we get

$$
\begin{aligned}
S(u) & =u^{-A_{0}}\left(\phi^{+}(u) u^{-A_{0}}\right)^{-1}\left(\phi^{-}(u)(-u)^{-A_{0}}\right)(-u)^{A_{0}} \\
& \sim u^{-A_{0}} \Upsilon(u)^{-1} \Upsilon(u)(-u)^{A_{0}} \\
& =u^{-A_{0}}(-u)^{A_{0}}
\end{aligned}
$$

which is equal to $\exp \left( \pm \pi \iota A_{0}\right)$ depending on whether $\operatorname{Im}(u) \lessgtr 0$.

Note that, if $\left[A_{0}, A(u)\right]=0$, it follows by (4.7) and (4.8) that

$$
\begin{aligned}
S(z) & =\prod_{n \geq 1}^{\leftarrow} A(u+n) e^{-A_{0} / n} A(u) \prod_{n \geq 1}^{\rightarrow} A(u-n) e^{A_{0} / n} \\
& =\cdots A(u+2) A(u+1) A(u) A(u-1) A(u-2) \cdots
\end{aligned}
$$

where the last expression is understood as $\lim _{n \rightarrow \infty} A(u+n) \cdots A(u+1) A(u) A(u-1)$ $\cdots A(u-n)$.

4.9. Example. The following basic example illustrates the constructions given above and plays an important role in the computations below. Consider the scalar difference equation

$$
\phi(u+1)=\frac{u-a}{u-b} \phi(u)
$$

with coefficient matrix $A(u)=\frac{u-a}{u-b}$. The canonical fundamental solutions $\phi^{ \pm}$are given by

$$
\phi^{+}(u)=\frac{\Gamma(u-a)}{\Gamma(u-b)} \quad \text { and } \quad \phi^{-}(u)=\frac{\Gamma(1-u+b)}{\Gamma(1-u+a)} .
$$

The connection matrix $S(u)$ is therefore equal to

$$
S(u)=\frac{e^{2 \pi \iota u}-e^{2 \pi \iota a}}{e^{2 \pi \iota u}-e^{2 \pi \iota b}} \cdot e^{\pi \iota(b-a)},
$$

where we used [52, Section 12.14]

$$
\Gamma(u) \Gamma(1-u)=\pi / \sin (\pi u) .
$$


4.10. Inverse problem. Fix now $A_{0} \in \operatorname{End}(V)$, and let $S$ be a rational function of $z=e^{2 \pi \iota u}$ such that $S(\infty)=e^{\pi \iota A_{0}}$ and $S(0)=e^{-\pi \iota A_{0}}$. We now discuss the problem of finding a coefficient matrix $A(u)$ of the form $1+A_{0} u^{-1}+\cdots$, such that $S(z)$ is the connection matrix of the system (4.1) determined by $A(u)$.

This may be phrased as the following factorization problem: find two meromorphic functions $\phi^{ \pm}(u): \mathbb{C} \rightarrow \operatorname{End}(V)$ such that

(a) $\phi^{ \pm}(u)$ are holomorphic and invertible for $\pm \operatorname{Re}(u) \gg 0$.

(b) $\phi^{ \pm}$possesses an asymptotic expansion of the form

$$
\phi^{ \pm}(u) \sim\left(1+H_{0}^{ \pm} u^{-1}+H_{1}^{ \pm} u^{-2}+\cdots\right) \cdot( \pm u)^{A_{0}}
$$

valid in any right (resp. left) half-plane.

(c) $S\left(e^{2 \pi \iota u}\right)=\phi^{+}(u)^{-1} \phi^{-}(u)$.

Once such a factorization is found, the coefficient matrix can be reconstructed as

$$
A(u)=\phi^{-}(u+1) \phi^{-}(u)^{-1}=\phi^{+}(u+1) \phi^{+}(u)^{-1},
$$

which is a rational function of $u$ since its poles are contained in a strip $|\operatorname{Re} u|<a$ by (a), and it tends to 1 as $u \rightarrow \infty$ by (b). By uniqueness, the canonical fundamental solutions of the difference equation defined by $A(u)$ are $\phi^{ \pm}$, so that its connection matrix is $S$ by (c).

The above factorization, and therefore the inverse problem do not always admit a solution. We refer the reader to [38] for several interesting cases when a solution can be obtained. In 4.14 4.16, we shall restrict our attention to the abelian case, that is, assume that $[S(z), S(w)]=0$. In this case a solution always exists, and it can be written explicitly in terms of Gamma functions.

4.11. Uniqueness of the factorization. Assume that $\phi_{1}^{ \pm}(u), \phi_{2}^{ \pm}(u)$ satisfy the properties (a)-(c) of 4.10, and set

$$
G(u)=\phi_{2}^{+}(u) \phi_{1}^{+}(u)^{-1}=\phi_{2}^{-}(u) \phi_{1}^{-}(u)^{-1} .
$$

$G$ is meromorphic and by (b) tends to 1 as $u \rightarrow \infty$, so that $G$ is a rational function. Thus, $\phi_{2}^{ \pm}(u)=G(u) \phi_{1}^{ \pm}(u)$, and the corresponding coefficient matrices are related by the isomonodromic transformation

$$
A_{\phi_{2}}(u)=\phi_{2}^{ \pm}(u+1) \phi_{2}^{ \pm}(u)^{-1}=G(u+1) A_{\phi_{1}}(u) G(u)^{-1} .
$$

The following gives a sufficient condition for the uniqueness of $A$. Call two subsets $B_{1}, B_{2} \subset \mathbb{C}$ non-congruent if $b_{1}-b_{2} \notin \mathbb{Z}_{\neq 0}$ for any $b_{1} \in B_{1}, b_{2} \in B_{2}$. We shall also say that $B \subset \mathbb{C}$ is non-congruent if it is non-congruent to itself.

Proposition. Let $A(u), A^{\prime}(u): \mathbb{C} \rightarrow G L(V)$ be rational functions of the form $1+A_{0} u^{-1}+\cdots$, and assume that the connection matrices of the difference equations determined by $A$ and $A^{\prime}$ are equal. Let $\mathcal{P}, \mathcal{Z} \subset \mathbb{C}$ (resp. $\left.\mathcal{P}^{\prime}, \mathcal{Z}^{\prime}\right)$ be the poles of $A(u), A(u)^{-1}$ (resp. $\left.A^{\prime}(u), A^{\prime}(u)^{-1}\right)$. If the pairs

$$
(\mathcal{Z}, \mathcal{P}), \quad\left(\mathcal{Z}^{\prime}, \mathcal{P}^{\prime}\right), \quad\left(\mathcal{Z}, \mathcal{Z}^{\prime}\right), \quad\left(\mathcal{P}, \mathcal{P}^{\prime}\right)
$$

are non-congruent, then $A=A^{\prime}$.

Proof. By the foregoing, there exists a rational function $G(u): \mathbb{C} \rightarrow G L(V)$ with $G(\infty)=1$, and such that $A^{\prime}(u)=G(u+1) A(u) G(u)^{-1}$. Thus, $G(u+1)=$ $A^{\prime}(u) G(u) A(u)^{-1}$ so that, for any $n \in \mathbb{Z}_{>0}$,

$$
G(u)=A^{\prime}(u-1) \cdots A^{\prime}(u-n) G(u-n) A(u-n)^{-1} \cdots A(u-1)^{-1} .
$$


Since $G(u)$ is regular for $\operatorname{Re}(u) \ll 0$, this implies that the poles of $G$ are contained in $\left(\mathcal{P}^{\prime} \cup \mathcal{Z}\right)+\mathbb{Z}_{>0}$. Similarly, $G(u)=A^{\prime}(u)^{-1} G(u+1) A(u)$, so that for any $n \in \mathbb{N}$

$$
G(u)=A^{\prime}(u)^{-1} \cdots A^{\prime}(u+n-1)^{-1} G(u+n) A(u+n-1) \cdots A(u),
$$

implying that the poles of $G$ are contained in $\left(\mathcal{Z}^{\prime} \cup \mathcal{P}\right)-\mathbb{N}$. The non-congruence assumption then implies that $G$ has no poles, and hence $G \equiv 1$ and $A^{\prime}=A$ as claimed.

4.12 .

Lemma. Let $M: \mathbb{C} \rightarrow G L(V)$ be a rational function such that

$$
[M(u), M(v)]=0
$$

for any $u, v \in \mathbb{C}$. Then, the semisimple and unipotent components $M_{S}, M_{U}: \mathbb{C} \rightarrow$ $G L(V)$ of the Jordan decomposition of $M$ are rational functions.

Proof. Let $\mathfrak{a} \subset \operatorname{End}(V)$ be the span of $\{M(u) \mid u \in \mathbb{C}\}$. By assumption, $\mathfrak{a}$ is an abelian Lie subalgebra of $\operatorname{End}(V)$, so that $V=\bigoplus_{\lambda \in \mathfrak{a}^{*}} V_{\lambda}$, where $V_{\lambda}$ is the generalized eigenspace

$$
V_{\lambda}=\left\{v \in V \mid(x-\lambda(x))^{N} v=0, \forall x \in \mathfrak{a}, N \gg 0\right\} .
$$

Set $M_{S}^{\prime}(u)=\sum_{\lambda} \mathbf{1}_{\lambda} \circ \lambda(M(u))$, where $\mathbf{1}_{\lambda}: V \rightarrow V_{\lambda}$ is the projection operator, and $M_{U}^{\prime}(u)=M_{S}^{\prime}(u)^{-1} M(u)$. By construction, $M_{S}^{\prime}(u), M_{U}^{\prime}(u)$ commute, are semisimple and unipotent, respectively, and satisfy $M(u)=M_{S}^{\prime}(u) M_{U}^{\prime}(u)$. By uniqueness of the Jordan decomposition $M_{S}=M_{S}^{\prime}$ and $M_{U}=M_{U}^{\prime}$, and the conclusion follows since $M_{S}^{\prime}$ and $M_{U}^{\prime}$ are rational functions.

4.13. Poles of abelian matrix functions. Retain the notation of Section 4.12 , and let $\mathcal{P}, \mathcal{P}_{S}, \mathcal{P}_{U}$ be the sets of poles of $M(z), M_{S}(z), M_{U}(z)$, and $\mathcal{Z}, \mathcal{Z}_{S}, \mathcal{Z}_{U}$ those of $M(z)^{-1}, M_{S}(z)^{-1}, M_{U}(z)^{-1}$, respectively.

Lemma. The following holds:

(i) $\mathcal{P}=\mathcal{P}_{S} \cup \mathcal{P}_{U}$ and $\mathcal{Z}=\mathcal{Z}_{S} \cup \mathcal{Z}_{U}$.

(ii) $\mathcal{P}_{U}=\mathcal{Z}_{U}$.

(iii) The eigenvalues of $M(z)$ are rational functions of $z$, and $\mathcal{P}_{S}$ (resp. $\mathcal{Z}_{S}$ ) consist of the poles (resp. zeros) of those eigenvalues.

Proof. (ii) follows from $M_{U}(z)^{ \pm 1}=\exp \left( \pm m_{U}(z)\right)$, with $m_{U}=\log \left(M_{U}(z)\right)$. (i) and (iii) follow by choosing a basis of $V$ in which $M_{S}(z)$ are all diagonal and $M_{U}(z)$ is strictly upper triangular.

4.14. Fix $A_{0} \in \operatorname{End}(V)$, and let $S: \mathbb{C} \rightarrow G L(V)$ be a rational function such that

- $S(\infty)=\exp \left(\pi \iota A_{0}\right)=S(0)^{-1}$;

- $[S(z), S(w)]=0$

- $\left[A_{0}, S(z)\right]=0$.

To factorize $S(z)$, we shall need to choose logarithms of its poles and zeros. Specifically, apply Section 4.13 to $M(z)=S(z)$ and set

$$
\mathcal{Z}_{S}=\left\{\alpha_{i}\right\}_{i \in I}, \quad \mathcal{P}_{S}=\left\{\beta_{j}\right\}_{j \in J}, \quad \mathcal{P}_{U}=\left\{\delta_{k}\right\}_{k \in K}=\mathcal{Z}_{U} .
$$

Let $(\lambda(z), \mu)$ be a joint eigenvalue of $\left(S(z), A_{0}\right)$. Then,

$$
\lambda(z)=e^{\pi \iota \mu} \prod_{i \in I^{\prime}, j \in J^{\prime}}\left(z-\alpha_{i}\right)^{n_{i}} /\left(z-\beta_{j}\right)^{m_{j}}
$$


for some $I^{\prime} \subset I, J^{\prime} \subset J$, and $n_{i}, m_{j} \in \mathbb{Z}_{>0}$. Since $S(\infty)=S(0)^{-1}$, we get $\prod \beta_{j}^{m_{j}} \alpha_{i}^{-n_{i}}=e^{2 \pi \iota \mu}$. Choose complex numbers $\left\{a_{i}^{(\lambda, \mu)}\right\}_{i \in I^{\prime}}$ and $\left\{b_{j}^{(\lambda, \mu)}\right\}_{j \in J^{\prime}}$ such that $e^{2 \pi \iota a_{i}^{(\lambda, \mu)}}=\alpha_{i}, e^{2 \pi \iota b_{j}^{(\lambda, \mu)}}=\beta_{j}$, and

$$
\sum_{j} m_{j} b_{j}^{(\lambda, \mu)}-\sum_{i} n_{i} a_{i}^{(\lambda, \mu)}=\mu .
$$

Let $\left\{\delta_{k}\right\}_{k \in K^{\prime}}$ be the poles of the restriction of $S_{U}(z)$ to the generalized eigenspace of $S(z)$ corresponding to $(\lambda, \mu)$, and choose $\left\{d_{k}^{(\lambda, \mu)}\right\}_{k \in \widetilde{K}}$ such that $e^{2 \pi \iota d_{k}^{(\lambda, \mu)}}=\delta_{k}$. Finally, set

$$
\begin{gathered}
\left\{a_{i}\right\}_{i \in \widetilde{I}}=\bigcup_{(\lambda, \mu)}\left\{a_{i}^{(\lambda, \mu)}\right\}_{i \in I^{\prime}}, \quad\left\{b_{j}\right\}_{j \in \widetilde{J}}=\bigcup_{(\lambda, \mu)}\left\{b_{j}^{(\lambda, \mu)}\right\}_{j \in J^{\prime}} \\
\left\{d_{k}\right\}_{k \in \widetilde{K}}=\bigcup_{(\lambda, \mu)}\left\{d_{k}^{(\lambda, \mu)}\right\}_{k \in K^{\prime}} .
\end{gathered}
$$

Theorem. For any consistent choice as above, there is a rational function $A: \mathbb{C} \rightarrow$ $G L(V)$ such that

- $A=1+A_{0} u^{-1}+\cdots$;

- $[A(u), A(v)]=0$;

- the connection matrix of the system defined by $A(u)$ is $S(z)$;

- the poles of $A(u)$ (resp. $A(u)^{-1}$ ) are $\left\{b_{j}\right\} \cup\left\{d_{k}\right\}$ (resp. $\left\{a_{i}\right\} \cup\left\{d_{k}\right\}$ ).

Moreover, if none of the sets $\left\{a_{i}\right\},\left\{b_{j}\right\},\left\{d_{k}\right\}$ are congruent to each other, or to themselves, $A$ is unique.

Proof. The uniqueness of $A(u)$ follows from Proposition 4.11, To prove the existence of $A(u)$, let $A_{0}=A_{0}^{S}+A_{0}^{N}$ be the Jordan decomposition of $A_{0}$. Since

$$
S_{S}(\infty)=e^{\pi \iota A_{0}^{S}}=S_{S}(0)^{-1} \quad \text { and } \quad S_{U}(\infty)=e^{\pi \iota A_{0}^{N}}=S_{U}(0)^{-1},
$$

it suffices to treat the cases when $S(z)$ is semisimple and unipotent separately, which we do in 4.15 and 4.16, respectively.

4.15. Semisimple case. Assume that $A_{0}$ and $S(z)$ are semisimple, let $\mathfrak{a} \subset \operatorname{End}(V)$ be the abelian Lie algebra spanned by $A_{0}$ and $S(w), w \in \mathbb{P}^{1}$, and decompose $V=\bigoplus_{\lambda \in \mathfrak{a}^{*}} V_{\lambda}$ as the direct sum of weight spaces under $\mathfrak{a}$. Since it suffices to factorize $S(z)$ on each $V_{\lambda}$, the problem reduces to the scalar case. We may therefore assume that

$$
A_{0} \in \mathbb{C} \quad \text { and } S(z)=e^{\pi \iota A_{0}} \prod_{i} \frac{z-\alpha_{i}}{z-\beta_{i}},
$$

where $\alpha_{i}, \beta_{i}$ are such that $\prod_{i} \alpha_{i} \beta_{i}^{-1}=e^{-2 \pi \iota A_{0}}$, so that $S(0)=e^{-\pi \iota A_{0}}$. Let $a_{i}, b_{i} \in$ $\mathbb{C}$ be such that

$$
e^{2 \pi \iota a_{i}}=\alpha_{i}, \quad e^{2 \pi \iota b_{i}}=\beta_{i}, \quad \sum_{i} b_{i}-a_{i}=A_{0} .
$$

Then, by Example 4.9, the factorization problem is solved by

$$
\phi^{+}=\prod_{i} \frac{\Gamma\left(u-a_{i}\right)}{\Gamma\left(u-b_{i}\right)} \quad \text { and } \quad \phi^{-}=\prod_{i} \frac{\Gamma\left(1-u+b_{i}\right)}{\Gamma\left(1-u+a_{i}\right)} .
$$

The corresponding coefficient matrix is

$$
A(u)=\phi^{+}(u+1) \phi^{+}(u)^{-1}=\prod_{i} \frac{u-a_{i}}{u-b_{i}} .
$$


4.16. Unipotent case. If $A_{0}$ is nilpotent, and $S(z)$ unipotent, taking logarithms reduces the factorization problem to finding meromorphic functions $\varphi^{ \pm}: \mathbb{C} \rightarrow$ $\operatorname{End}(V)$ such that

(a) $\varphi^{ \pm}$is holomorphic for $\pm \operatorname{Re}(u) \gg 0$.

(b) $\varphi^{ \pm}(u)$ has an asymptotic expansion of the form

$$
\varphi^{ \pm}(u) \sim A_{0} \log ( \pm u)+h_{0}^{ \pm} u^{-1}+h_{1}^{ \pm} u^{-2}+\cdots
$$

valid in any right (resp. left) half-plane.

(c) $\varphi^{-}(u)-\varphi^{+}(u)=\log \left(S\left(e^{2 \pi \iota u}\right)\right)$.

Since $S(\infty)=e^{\pi \iota A_{0}}=S(0)^{-1}$, it follows that

$$
\log (S(z))=\pi \iota A_{0}+\sum_{k} \sum_{r \geq 1} \frac{C_{r, k}}{\left(z-\delta_{k}\right)^{r}}
$$

where $\left\{\delta_{k}\right\}$ are the poles of $S(z)$, and $C_{r, k} \in \operatorname{End}(V)$ are such that

$$
\sum C_{r, k}\left(-\delta_{k}\right)^{-r}=-2 \pi \iota A_{0} .
$$

To obtain an additive factorization of $\log (S(z))$, we first determine meromorphic functions $\varphi_{r}^{ \pm}(u, d)$ for any $r \geq 1$ and $d \in \mathbb{C}$, which are holomorphic for $\pm \operatorname{Re}(u) \gg 0$, and such that

$$
\varphi_{r}^{-}(u, d)-\varphi_{r}^{+}(u, d)=\frac{1}{(z-\delta)^{r}},
$$

where $z=e^{2 \pi \iota u}$ and $\delta=e^{2 \pi \iota d}$.

Set $\Psi^{+}(u)=\Gamma^{\prime}(u) / \Gamma(u)$ and $\Psi^{-}(u)=\Gamma^{\prime}(1-u) / \Gamma(1-u)$. Taking logarithmic derivatives in $\Gamma(1-u) \Gamma(u)=\pi / \sin (\pi u)$, we get

$$
\Psi^{-}(u)-\Psi^{+}(u)=\pi \cot (\pi u)=\pi \iota\left(\frac{2}{z-1}+1\right)
$$

so that the functions

$$
\varphi_{1}^{ \pm}(u, d)=\frac{1}{2 \delta}\left(\frac{\Psi^{ \pm}(u-d)}{\pi \iota} \pm \frac{1}{2}\right)
$$

solve (4.12) for $r=1$. Next, if $\varphi_{r}^{ \pm}(u, d)$ solve (4.12) for a given $r$, then

$$
\begin{aligned}
\partial_{u} \varphi_{r}^{-}(u, d)-\partial_{u} \varphi_{r}^{+}(u, d) & =-\frac{2 \pi \iota r z}{(z-\delta)^{r+1}} \\
& =-2 \pi \iota r\left(\frac{\delta}{(z-\delta)^{r+1}}+\frac{1}{(z-\delta)^{r}}\right)
\end{aligned}
$$

so that $\varphi_{r+1}^{ \pm}(u, d)=-\frac{1}{\delta}\left(\frac{\partial_{u}}{2 \pi \iota r}+1\right) \varphi_{r}^{ \pm}(u, d)$ solve (4.12) for $r+1$. We therefore recursively get

$$
\varphi_{r}^{ \pm}(u, d)=-\frac{(-\delta)^{-r}}{2} \prod_{k=1}^{r-1}\left(\frac{\partial_{u}}{2 \pi \iota k}+1\right)\left(\frac{\Psi^{ \pm}(u-d)}{\pi \iota} \pm \frac{1}{2}\right) .
$$

Since $\Psi^{ \pm}(u)=\log ( \pm u)+O\left(u^{-1}\right)$ in any right (resp. left) half-plane [52, Section 12.33], where $O\left(u^{-1}\right)$ denotes an element of $u^{-1} \mathbb{C}\left[\left[u^{-1}\right]\right]$, it follows that

$$
\varphi_{r}^{ \pm}(u, d) \sim-(-\delta)^{-r}\left(\frac{\log ( \pm u)}{2 \pi \iota} \pm \frac{1}{4}\right)+O\left(u^{-1}\right) .
$$


Choose now $d_{k} \in \mathbb{C}$ such that $e^{2 \pi \iota d_{k}}=\delta_{k}$, and set

$$
\varphi^{ \pm}(u)=\mp \frac{\pi \iota A_{0}}{2}+\sum_{k, r} \varphi_{r}^{ \pm}\left(u, d_{k}\right) C_{k, r}
$$

then $\varphi^{-}(u)-\varphi^{+}(u)=\log S(z)$ and, in any right (resp. left) half-plane,

$$
\begin{aligned}
\varphi^{ \pm} & \sim \mp \frac{\pi \iota A_{0}}{2}-\left(\frac{\log ( \pm u)}{2 \pi \iota} \pm \frac{1}{4}\right) \sum C_{k, r}\left(-\delta_{k}\right)^{-r}+O\left(u^{-1}\right) \\
& =\mp \frac{\pi \iota A_{0}}{2}+2 \pi \iota A_{0}\left(\frac{\log ( \pm u)}{2 \pi \iota} \pm \frac{1}{4}\right)+O\left(u^{-1}\right) \\
& =A_{0} \log ( \pm u)+O\left(u^{-1}\right),
\end{aligned}
$$

where we used (4.11), as required.

To determine the coefficient matrix of the difference equation, let $\Delta$ be the operator defined by $\Delta f(u)=f(u+1)-f(u)$. Since $\Delta \Psi^{ \pm}(u)=1 / u$, 4.13) yields

$$
\Delta \varphi_{r}^{ \pm}(u, d)=-\frac{(-\delta)^{-r}}{2 \pi \iota} \prod_{k=1}^{r-1}\left(\frac{\partial_{u}}{2 \pi \iota k}+1\right) \frac{1}{u-d}
$$

so that $A(u)=\exp \left(\Delta \varphi^{ \pm}\right)$is given by

$$
A(u)=\exp \left(-\frac{1}{2 \pi \iota} \sum_{k, r}\left(-\delta_{k}\right)^{-r} \prod_{k=1}^{r-1}\left(\frac{\partial_{u}}{2 \pi \iota k}+1\right) \frac{C_{k, r}}{u-d_{k}}\right) .
$$

\section{THE FUNCTOR $\Gamma$}

In this section, we construct a functor $\Gamma$ from a dense subcategory of $\mathcal{O}_{\text {int }}\left(Y_{\hbar}(\mathfrak{g})\right)$ to $\mathcal{O}_{\text {int }}\left(U_{q}(L \mathfrak{g})\right)$.

5.1. Non-congruent representations. We shall say that $V \in \mathcal{O}_{\text {int }}\left(Y_{\hbar}(\mathfrak{g})\right)$ is noncongruent if, for any $i \in \mathbf{I}$, the poles of $x_{i}^{+}(u)$ (resp. $x_{i}^{-}(u)$ ) are not congruent modulo $\mathbb{Z}$. Let $\mathcal{O}_{\text {int }}^{\text {NC }}\left(Y_{\hbar}(\mathfrak{g})\right)$ be the full subcategory of $\mathcal{O}_{\text {int }}\left(Y_{\hbar}(\mathfrak{g})\right)$ consisting of non-congruent representations.

5.2. Contours. By a Jordan curve $\mathcal{C}$, we shall mean a disjoint union of simple, closed curves in $\mathbb{C}$, the inner domains of which are pairwise disjoint. By definition, the inner (resp. outer) domain of $\mathcal{C}$ is the union (resp. intersection) of those of its connected components. If $\mathcal{C}$ is a Jordan curve, and $f$ a continuous function on $\mathcal{C}$, we shall set

$$
\oint_{\mathcal{C}} f(u) d u=\frac{1}{2 \pi \iota} \int_{\mathcal{C}} f(u) d u .
$$

The definition of $\Gamma$ relies upon the following contours of integration. Given $V \in \mathcal{O}_{\text {int }}\left(Y_{\hbar}(\mathfrak{g})\right)$, a weight $\mu \in \mathfrak{h}^{*}$ of $V$, and $i \in \mathbf{I}$, we shall denote by $\mathcal{C}_{i, \mu}^{ \pm} \subset \mathbb{C}$ a Jordan curve such that

(i) $\mathcal{C}_{i, \mu}^{ \pm}$encloses the poles of $x_{i}^{ \pm}(u)_{\mu}$.

(ii) $\mathcal{C}_{i, \mu}^{ \pm}$encloses no $\mathbb{Z}_{\neq 0}$-translate of the poles of $x_{i}^{ \pm}(u)_{\mu}$ and $x_{i}^{ \pm}(u)_{\mu \pm \alpha_{i}}$.

Note that such a curve exists for any $i \in \mathbf{I}$ and weight $\mu \in \mathfrak{h}^{*}$ if $V$ is non-congruent. 
5.3. Quantum loop operators. Given $V \in \mathcal{O}_{\text {int }}^{\text {NC }}\left(Y_{\hbar}(\mathfrak{g})\right)$, and a weight $\mu$ of $V$, we define the action of the generators of $U_{q}(L \mathfrak{g})$ on the $\mu$-weight space $V_{\mu}$ of $\Gamma(V)=V$ as follows.

(i) For any $h \in \mathfrak{h}$, set $\left(K_{h}\right)_{\mu}=q^{\mu(h)}$.

(ii) For any $i \in \mathbf{I}$, consider the abelian, additive difference equation

$$
\phi_{i}(u+1)_{\mu}=\xi_{i}(u)_{\mu} \phi_{i}(u)_{\mu}
$$

with coefficient matrix $\xi_{i}(u)_{\mu}=1+\hbar \xi_{i, 0} u^{-1}+\cdots$. Let $S_{i}(u)_{\mu}$ be its connection matrix, as defined in 4.8 , Set

$$
\Psi_{i}(z)_{\mu}=\left.S_{i}(u)_{\mu}\right|_{e^{2 \pi \iota u}=z},
$$

and let $\Psi_{i}(z)_{\mu}^{ \pm} \in \operatorname{End}\left(V_{\mu}\right)\left[\left[z^{\mp 1}\right]\right]$ be its Taylor expansions at $\infty$ and 0 , respectively.

To define the action of the remaining generators of $U_{q}(L \mathfrak{g})$, consider the fundamental solutions $\phi_{i}^{ \pm}(u)_{\mu}$ of (5.1), as defined in 4.2. Define $g_{i}^{ \pm}(u)_{\mu}: \mathbb{C} \rightarrow G L\left(V_{\mu}\right)$ by

$$
g_{i}^{-}(u)_{\mu}=\phi_{i}^{-}(u)_{\mu} \quad \text { and } \quad g_{i}^{+}(u)_{\mu}=\phi_{i}^{+}(u+1)_{\mu}^{-1} .
$$

Note that, by (4.7) and (4.8),

$$
\begin{aligned}
g_{i}^{-}(u)_{\mu} & =e^{-\gamma \hbar \xi_{i, 0}} \prod_{n \geq 1}^{\rightarrow} \xi_{i}(u-n)_{\mu} e^{\hbar \xi_{i, 0} / n}, \\
g_{i}^{+}(u)_{\mu} & =\left(\prod_{n \geq 1}^{\leftarrow} e^{-\hbar \xi_{i, 0} / n} \xi_{i}(u+n)_{\mu}\right) e^{\gamma \hbar \xi_{i, 0}} .
\end{aligned}
$$

(iii) Let $c_{i}^{ \pm} \in \mathbb{C}^{\times}$be scalars such that

$$
c_{i}^{-} c_{i}^{+}=d_{i} \Gamma\left(\hbar d_{i}\right)^{2} .
$$

For any $i \in \mathbf{I}$ and $k \in \mathbb{Z}$, the action of $\mathcal{X}_{i, k}^{ \pm}$as an operator $V_{\mu} \rightarrow V_{\mu \pm \alpha_{i}}$ is given by

$$
\left(\mathcal{X}_{i, k}^{ \pm}\right)_{\mu}=c_{i}^{ \pm} \oint_{\mathcal{C}_{i, \mu}^{ \pm}} e^{2 \pi \iota k u} g_{i}^{ \pm}(u)_{\mu \pm \alpha_{i}} x_{i}^{ \pm}(u)_{\mu} d u,
$$

where the contours $\mathcal{C}_{i, \mu}^{ \pm}$are chosen as in 5.2 .

Remark. The above definition implies that the generating functions

$$
\mathcal{X}_{i}^{ \pm}(z)_{\mu}^{+}=\sum_{k \geq 0} \mathcal{X}_{i, k}^{ \pm} z^{-k} \quad \text { and } \quad \mathcal{X}_{i}^{ \pm}(z)_{\mu}^{-}=-\sum_{k<0} \mathcal{X}_{i, k}^{ \pm} z^{-k}
$$

are the expansions at $z=\infty$ and $z=0$ of the rational function

$$
\mathcal{X}_{i}^{ \pm}(z)_{\mu}=c_{i}^{ \pm} \oint_{\mathcal{C}_{i, \mu}^{ \pm}}\left(\frac{z}{z-e^{2 \pi \iota u}}\right) g_{i}^{ \pm}(u)_{\mu \pm \alpha_{i}} x_{i}^{ \pm}(u)_{\mu} d u,
$$

where $z$ lies outside the contour $\exp \left(2 \pi \iota \mathcal{C}_{i, \mu}^{ \pm}\right)$.

Remark. The operator (5.6) is independent of the choice of the contour $\mathcal{C}_{i, \mu}^{ \pm}$satisfying the conditions of Section 5.2. Indeed, by (5.3), (5.4), and Lemma 3.7 the poles of $g_{i}^{ \pm}(u)_{\mu \pm \alpha_{i}}$ are contained in the $\mathbb{Z}_{\lessgtr 0}$-translates of the poles of $x_{i}^{ \pm}(u)_{\mu}$ and $x_{i}^{ \pm}(u)_{\mu \pm \alpha_{i}}$. In particular, $g_{i}^{ \pm}(u)$ is holomorphic inside $\mathcal{C}_{i, \mu}^{ \pm}$. 
It also follows that, for any finite collection of weights $\Xi \subset \mathfrak{h}^{*}$ of $V$, a contour $\mathcal{C}_{i}^{ \pm}$can be chosen which satisfies (i) and (ii) of Section 5.2 for all $\mu \in \Xi$.

5.4 .

\section{Theorem.}

(i) The above operators give rise to an action of $U_{q}(L \mathfrak{g})$ on $V$. They therefore define an exact, faithful functor

$$
\Gamma: \mathcal{O}_{\text {int }}^{\mathrm{NC}}\left(Y_{\hbar}(\mathfrak{g})\right) \longrightarrow \mathcal{O}_{\text {int }}\left(U_{q}(L \mathfrak{g})\right) .
$$

(ii) The functor $\Gamma$ is compatible with shift automorphisms. That is, for any $V \in \mathcal{O}_{\text {int }}^{\mathrm{NC}}\left(Y_{\hbar}(\mathfrak{g})\right)$ and $a \in \mathbb{C}$,

$$
\Gamma(V(a))=\Gamma(V)\left(e^{2 \pi \iota a}\right) .
$$

(iii) Let $\Pi \subset \mathbb{C}$ be a non-congruent subset such that $\Pi \pm \frac{1}{2} \hbar \subset \Pi$. Then, $\mathcal{O}_{\mathrm{int}}^{\Pi}\left(Y_{\hbar}(\mathfrak{g})\right)$ is a subcategory of $\mathcal{O}_{\mathrm{int}}^{\mathrm{NC}}\left(Y_{\hbar}(\mathfrak{g})\right)$, and $\Gamma$ restricts to a functor

$$
\Gamma_{\Pi}: \mathcal{O}_{\mathrm{int}}^{\Pi}\left(Y_{\hbar}(\mathfrak{g})\right) \longrightarrow \mathcal{O}_{\mathrm{int}}^{\Omega}\left(U_{q}(L \mathfrak{g})\right),
$$

where $\Omega=\exp (2 \pi \iota \Pi)$.

Proof. (i) The relations (QL0), (QL1), and (QL2) clearly hold. We prove (QL3) in 5.6. (QL4) in 5.7, and (QL5) in 5.9. Relation (QL6) is a consequence of Proposition 2.13 .

$A$ word on notation: The relations of $U_{q}(L \mathfrak{g})$ need to be verified on a given weight space. For notational convenience, we omit the subscript indicating the weight space in the proofs below. Moreover, the contours $\mathcal{C}_{i}^{ \pm}$are chosen to satisfy the conditions of Section 5.2 for all the weight spaces under consideration, consistently with Remark 5.3 .

(ii) is proved in 5.10

(iii) follows from Theorem 3.8 and the monodromy computation in 4.9

5.5. Set $\Gamma^{ \pm}(u)=\Gamma(1 \pm u)$, and note that the identity (4.9) reads

$$
\Gamma^{-}(u) \Gamma^{+}(u)=\frac{2 \pi \iota u}{e^{\pi \iota u}-e^{-\pi \iota u}} .
$$

Let $V \in \mathcal{O}_{\text {int }}\left(Y_{\hbar}(\mathfrak{g})\right), i, j \in \mathbf{I}$, and $a=\hbar d_{i} a_{i j} / 2$. We shall need the following proposition.

Proposition. Let $\mathcal{C}$ be a Jordan curve with interior domain $D, \Omega_{1}, \Omega_{2} \subset \mathbb{C}$ two open subsets with $\bar{D} \subset \Omega_{2}$, and $f: \Omega_{1} \times \Omega_{2} \rightarrow \operatorname{End}(V)$ a holomorphic function such that $\left[\xi_{i}(u), f(u, v)\right]=0$ for any $u, v$. Then, the following holds for any $\epsilon, \eta \in\{ \pm\}$.

(i) If $u \notin \bar{D} \pm \epsilon a$,

$$
\operatorname{Ad}\left(\xi_{i}(u)\right)^{ \pm 1} \oint_{\mathcal{C}} f(u, v) x_{j}^{\epsilon}(v) d v=\oint_{\mathcal{C}}\left(\frac{u-v+\epsilon a}{u-v-\epsilon a}\right)^{ \pm 1} f(u, v) x_{j}^{\epsilon}(v) d v .
$$

(ii) If $u \notin \bar{D} \pm \epsilon a-\eta \mathbb{Z}_{>0}$,

$$
\operatorname{Ad}\left(g_{i}^{\eta}(u)\right)^{ \pm 1} \oint_{\mathcal{C}} f(u, v) x_{j}^{\epsilon}(v) d v=\oint_{\mathcal{C}}\left(\frac{\Gamma^{\eta}(u-v-\epsilon a)}{\Gamma^{\eta}(u-v+\epsilon a)}\right)^{ \pm 1} f(u, v) x_{j}^{\epsilon}(v) d v
$$


(iii) If $u \notin \bar{D} \pm \epsilon a+\mathbb{Z}$,

$$
\begin{aligned}
& \operatorname{Ad}\left(S_{i}(u)\right)^{ \pm 1} \oint_{\mathcal{C}} f(u, v) x_{j}^{\epsilon}(v) d v=\oint_{\mathcal{C}}\left(\frac{q_{i}^{\epsilon a_{i j}} z-w}{z-q_{i}^{\epsilon a_{i j}} w}\right)^{ \pm 1} f(u, v) x_{j}^{\epsilon}(v) d v \\
& \text { where } z=e^{2 \pi \iota u} \text { and } w=e^{2 \pi \iota v} .
\end{aligned}
$$

Proof. (i) follows by left multiplying $(\mathcal{Y} 3)$ by $f(u, v)$ and integrating along $\mathcal{C}$.

(ii) A repeated application of (i) shows that, for any $n \geq 1$

$$
\begin{aligned}
\operatorname{Ad}\left(\xi_{i}(u+\eta n) \cdots \xi_{i}(u+\eta 1)\right)^{ \pm 1} & \oint_{\mathcal{C}} f(u, v) x_{j}^{\epsilon}(v) d v \\
& =\oint_{\mathcal{C}} \prod_{m=1}^{n}\left(\frac{u+\eta m-v+\epsilon a}{u+\eta m-v-\epsilon a}\right)^{ \pm 1} f(u, v) x_{j}^{\epsilon}(v) d v
\end{aligned}
$$

so long as $u \notin \bar{D} \pm \epsilon a-\{\eta 1, \ldots, \eta n\}$. The result then follows from (5.3) and (5.4), $\left[\hbar \xi_{i, 0}, x_{j}^{\epsilon}(v)\right]=\epsilon 2 a x_{j}^{\epsilon}(v)$, and the fact that, for any $\alpha, \beta \in \mathbb{C}$,

$$
e^{\gamma(\alpha-\beta)} \prod_{m=1}^{n} \frac{m+\alpha}{m+\beta} e^{-\frac{\alpha-\beta}{m}} \longrightarrow \frac{\beta}{\alpha} \frac{\Gamma(\beta)}{\Gamma(\alpha)}=\frac{\Gamma^{+}(\beta)}{\Gamma^{+}(\alpha)}=\frac{\Gamma^{-}(-\beta)}{\Gamma^{-}(-\alpha)} \text { as } n \rightarrow \infty .
$$

(iii) follows from (i) and (ii), the fact that

$$
S_{i}(u)=g_{i}^{+}(u) \xi_{i}(u) g_{i}^{-}(u),
$$

and (5.8).

5.6. Proof of (QL3). We shall check that

$$
z\left(\operatorname{Ad}\left(\Psi_{i}(z)\right) \mathcal{X}_{j, \ell}^{ \pm}-q_{i}^{ \pm a_{i j}} \mathcal{X}_{j, \ell}^{ \pm}\right)=q_{i}^{ \pm a_{i j}} \operatorname{Ad}\left(\Psi_{i}(z)\right) \mathcal{X}_{j, \ell+1}^{ \pm}-\mathcal{X}_{j, \ell+1}^{ \pm} .
$$

The relation (QL3) then follows by expanding around $z=\infty$ and $z=0$.

Since (5.10) is an equality of rational functions, it suffices to prove it when $z$ lies outside of $\exp \left(2 \pi \iota \mathcal{C}_{j}^{ \pm}\right)$. Since $g_{j}^{ \pm}$is holomorphic inside $\mathcal{C}_{j}^{ \pm}$by Remark [5.3, Proposition 5.5 implies that, for any $k \in \mathbb{Z}$,

$$
\operatorname{Ad}\left(\Psi_{i}(z)\right) \mathcal{X}_{j, k}^{ \pm}=c_{j}^{ \pm} \oint_{\mathcal{C}_{j}^{ \pm}} w^{k} \frac{q_{i}^{ \pm a_{i j}} z-w}{z-q_{i}^{ \pm a_{i j}} w} g_{j}^{ \pm}(v) x_{j}^{ \pm}(v) d v
$$

where $w=\exp (2 \pi \iota v)$. The left-hand side of (5.10) is therefore equal to

$$
c_{j}^{ \pm} \oint_{\mathcal{C}_{j}^{ \pm}} z w^{\ell}\left(\frac{q_{i}^{ \pm a_{i j}} z-w}{z-q_{i}^{ \pm a_{i j}} w}-q_{i}^{ \pm a_{i j}}\right) g_{j}^{ \pm}(v) x_{j}^{ \pm}(v) d v
$$

and the right-hand side to

$$
c_{j}^{ \pm} \oint_{\mathcal{C}_{j}^{ \pm}} w^{\ell+1}\left(q_{i}^{ \pm a_{i j}} \frac{q_{i}^{ \pm a_{i j}} z-w}{z-q_{i}^{ \pm a_{i j}} w}-1\right) g_{j}^{ \pm}(v) x_{j}^{ \pm}(v) d v .
$$


5.7. Proof of (QL4). We shall prove (QL4) in the form

$$
\mathcal{X}_{i, k+1}^{ \pm} \mathcal{X}_{j, \ell}^{ \pm}-q_{i}^{ \pm a_{i j}} \mathcal{X}_{i, k}^{ \pm} \mathcal{X}_{j, \ell+1}^{ \pm}=q_{i}^{ \pm a_{i j}} \mathcal{X}_{j, \ell}^{ \pm} \mathcal{X}_{i, k+1}^{ \pm}-\mathcal{X}_{j, \ell+1}^{ \pm} \mathcal{X}_{i, k}^{ \pm}
$$

Set $a=\hbar d_{i} a_{i j} / 2$, so that $\exp (2 \pi \iota a)=q_{i}^{a_{i j}}$. By Proposition 5.5 ,

$$
\begin{aligned}
& \oint_{\mathcal{C}_{i}^{ \pm}}\left(e^{2 \pi \iota(k+1) u} e^{2 \pi \iota \ell v}-q_{i}^{ \pm a_{i j}} e^{2 \pi \iota k u} e^{2 \pi \iota(\ell+1) v}\right) g_{i}^{ \pm}(u) x_{i}^{ \pm}(u) d u \cdot g_{j}^{ \pm}(v) \\
= & g_{j}^{ \pm}(v) \cdot \oint_{\mathcal{C}_{i}^{ \pm}} e^{2 \pi \iota(k u+\ell v)}\left(e^{2 \pi \iota u}-e^{2 \pi \iota(v \pm a)}\right) \frac{\Gamma^{ \pm}(v-u \pm a)}{\Gamma^{ \pm}(v-u \mp a)} g_{i}^{ \pm}(u) x_{i}^{ \pm}(u) d u
\end{aligned}
$$

so long as $v \notin \bar{D}_{i}^{ \pm} \mp a \mp \mathbb{Z}_{>0}$. As a function of $v$, the integrand on the right-hand side of (5.12) is regular on $\mathcal{C}_{i}^{ \pm} \mp a \mp \mathbb{Z}_{>0}$ since the (simple) poles of $\Gamma^{ \pm}(v-u \pm a)$ are canceled by the zeros of $\left(e^{2 \pi \iota u}-e^{2 \pi \iota(v \pm a)}\right)$. It follows that (5.12) holds for any $v$.

Right multiplying the above by $x_{j}^{ \pm}(v)$ and integrating along $v \in \mathcal{C}_{j}^{ \pm}$then shows that the left-hand side of (5.11) is equal to

$$
\begin{aligned}
c_{i}^{ \pm} c_{j}^{ \pm} \oint_{\mathcal{C}_{j}^{ \pm}} \oint_{\mathcal{C}_{i}^{ \pm}} e^{2 \pi \iota(k u+\ell v)}\left(e^{2 \pi \iota u}-\right. & \left.e^{2 \pi \iota(v \pm a)}\right) \\
& \cdot \frac{\Gamma^{ \pm}(v-u \pm a)}{\Gamma^{ \pm}(v-u \mp a)} g_{i}^{ \pm}(u) g_{j}^{ \pm}(v) x_{i}^{ \pm}(u) x_{j}^{ \pm}(v) d u d v .
\end{aligned}
$$

Similarly, the right-hand side is equal to

$$
\begin{aligned}
c_{i}^{ \pm} c_{j}^{ \pm} \oint_{\mathcal{C}_{j}^{ \pm}} \oint_{\mathcal{C}_{i}^{ \pm}} e^{2 \pi \iota(k u+\ell v)}\left(e^{2 \pi \iota(u \pm a)}-e^{2 \pi \iota v}\right) \\
\cdot \frac{\Gamma^{ \pm}(u-v \pm a)}{\Gamma^{ \pm}(u-v \mp a)} g_{i}^{ \pm}(u) g_{j}^{ \pm}(v) x_{j}^{ \pm}(v) x_{i}^{ \pm}(u) d u d v
\end{aligned}
$$

By $(\mathcal{Y} 4)$, the latter expression may be rewritten as

$$
\begin{aligned}
c_{i}^{ \pm} c_{j}^{ \pm} \oint_{\mathcal{C}_{j}^{ \pm}} \oint_{\mathcal{C}_{i}^{ \pm}} e^{2 \pi \iota(k u+\ell v)} & \left(e^{2 \pi \iota(u \pm a)}-e^{2 \pi \iota v}\right) \\
\cdot & \frac{v-u \pm a}{v-u \mp a} \frac{\Gamma^{ \pm}(u-v \pm a)}{\Gamma^{ \pm}(u-v \mp a)} g_{i}^{ \pm}(u) g_{j}^{ \pm}(v) x_{i}^{ \pm}(u) x_{j}^{ \pm}(v) d u d v
\end{aligned}
$$

since the contribution of the terms $\left[x_{i, 0}^{ \pm}, x_{j}^{ \pm}(v)\right]$ and $\left[x_{i}^{ \pm}(u), x_{j, 0}^{ \pm}(v)\right]$ to the double integral is zero given that $\left(e^{2 \pi \iota(u \pm a)}-e^{2 \pi \iota v}\right) \Gamma^{ \pm}(u-v \pm a)$ is holomorphic in $D_{i} \times D_{j}$.

(QL4) now follows from the fact that $\Gamma^{+}(x)=\Gamma^{-}(-x)$ and (5.8).

5.8. We shall need the following result to show that relation (QL5) holds.

Lemma. Let $\mathcal{C} \subset \mathbb{C}$ be a Jordan curve with interior domain $D, \xi(u)$ a meromorphic function with no singularities on $\mathcal{C}$, and $f(u, v)$ a meromorphic function with no singularities in $\bar{D} \times \bar{D}$. Then,

$$
\oint_{\mathcal{C}} \oint_{\mathcal{C}} f(u, v) \frac{\xi(u)-\xi(v)}{u-v} d u d v=-\oint_{\mathcal{C}} f(u, u) \xi(u) d u .
$$


Proof. Let $\mathcal{C}_{<}$be a small deformation of $\mathcal{C}$ contained in $\mathcal{C}$. Then,

$$
\begin{aligned}
\oint_{\mathcal{C}} \oint_{\mathcal{C}} f(u, v) \frac{\xi(u)-\xi(v)}{u-v} d u d v & =\oint_{\mathcal{C}}\left(\oint_{\mathcal{C}_{<}} f(u, v) \frac{\xi(u)-\xi(v)}{u-v} d u\right) d v \\
& =\oint_{\mathcal{C}}\left(\oint_{\mathcal{C}_{<}} f(u, v) \frac{\xi(u)}{u-v} d u\right) d v \\
& =\oint_{\mathcal{C}_{<}} \xi(u)\left(\oint_{\mathcal{C}} \frac{f(u, v)}{u-v} d v\right) d u \\
& =-\oint_{\mathcal{C}_{<}} \xi(u) f(u, u) d u .
\end{aligned}
$$

5.9. Proof of (QL5). We have

$$
\begin{aligned}
{\left[\mathcal{X}_{i, k}^{+}, \mathcal{X}_{j, \ell}^{-}\right] } & =c_{i}^{+} c_{j}^{-} \oint_{\mathcal{C}_{i}^{+}} \oint_{\mathcal{C}_{j}^{-}} e^{2 \pi \iota k u} e^{2 \pi \iota \ell v} g_{i}^{+}(u) x_{i}^{+}(u) g_{j}^{-}(v) x_{j}^{-}(v) d u d v \\
& -c_{i}^{+} c_{j}^{-} \oint_{\mathcal{C}_{j}^{-}} \oint_{\mathcal{C}_{i}^{+}} e^{2 \pi \iota k u} e^{2 \pi \iota \ell v} g_{j}^{-}(v) x_{j}^{-}(v) g_{i}^{+}(u) x_{i}^{+}(u) d u d v
\end{aligned}
$$

We wish to apply Proposition 5.5 to permute $x_{i}^{+}(u)$ and $g_{j}^{-}(v)$ in the first integral, and $x_{j}^{-}(v)$ and $g_{i}^{+}(u)$ in the second. This cannot be done directly, however, since $\mathcal{C}_{j}^{-} \ni v$ may lie inside $\bar{D}_{i}^{+}-a+\mathbb{Z}_{>0}$, where $a=\hbar d_{i} a_{i j} / 2$, and $\mathcal{C}_{i}^{+} \ni u$ may lie inside $\bar{D}_{j}^{-}+a-\mathbb{Z}_{>0}$.

To circumvent this issue, let $\delta \in \mathbb{C}$ and consider the shifted integral

$$
\begin{aligned}
I(\delta)=c_{i}^{+} c_{j}^{-} & \oint_{\mathcal{C}_{i}^{+}} \oint_{\mathcal{C}_{j}^{-}} e^{2 \pi \iota k u} e^{2 \pi \iota \ell v} g_{i}^{+}(u+\delta) x_{i}^{+}(u) g_{j}^{-}(v-\delta) x_{j}^{-}(v) d u d v \\
& -c_{i}^{+} c_{j}^{-} \oint_{\mathcal{C}_{j}^{-}} \oint_{\mathcal{C}_{i}^{+}} e^{2 \pi \iota k u} e^{2 \pi \iota \ell v} g_{j}^{-}(v-\delta) x_{j}^{-}(v) g_{i}^{+}(u+\delta) x_{i}^{+}(u) d v d u
\end{aligned}
$$

$I(\delta)$ is holomorphic in a disk $|\delta|<R$, where $R$ is such that $g_{i}^{+}$and $g_{j}^{-}$are holomorphic on $\bar{D}_{i}^{+}+\delta^{\prime}$ and $\bar{D}_{j}^{-}-\delta^{\prime}$ for any $\delta^{\prime}$ such that $\left|\delta^{\prime}\right|<R$, respectively. Moreover, if $\mathcal{C}_{i}^{+}, \mathcal{C}_{j}^{-}$are small enough, there is an $r<R$ such that if $|\delta|>r, \bar{D}_{j}^{-}-\delta$ is disjoint from $\bar{D}_{i}^{+}-a+\mathbb{Z}_{>0}$, and $\bar{D}_{i}^{+}+\delta$ is disjoint from $\bar{D}_{j}^{-}+a-\mathbb{Z}_{>0}$.

Assuming that $r<|\delta|<R$, we may now apply Proposition 5.5 to find that $I(\delta)$ is equal to

$$
\begin{aligned}
c_{i}^{+} c_{j}^{-} & \oint_{\mathcal{C}_{i}^{+}} \oint_{\mathcal{C}_{j}^{-}} e^{2 \pi \iota k u} e^{2 \pi \iota \ell v} g_{i}^{+}(u+\delta) g_{j}^{-}(v-\delta) \frac{\Gamma^{-}(v-\delta-u+a)}{\Gamma^{-}(v-\delta-u-a)} x_{i}^{+}(u) x_{j}^{-}(v) d u d v \\
-c_{i}^{+} c_{j}^{-} & \oint_{\mathcal{C}_{j}^{-}} \oint_{\mathcal{C}_{i}^{+}} e^{2 \pi \iota k u} e^{2 \pi \iota \ell v} g_{j}^{-}(v-\delta) g_{i}^{+}(u+\delta) \frac{\Gamma^{+}(u+\delta-v-a)}{\Gamma^{+}(u+\delta-v+a)} x_{j}^{-}(v) x_{i}^{+}(u) d v d u
\end{aligned}
$$


Since $\Gamma^{+}(x)=\Gamma^{-}(x)$, this yields

$$
\begin{aligned}
\frac{I(\delta)}{c_{i}^{+} c_{j}^{-}}= & \oint_{\mathcal{C}_{i}^{+}} \oint_{\mathcal{C}_{j}^{-}} e^{2 \pi \iota k u} e^{2 \pi \iota \ell v} g_{i}^{+}(u+\delta) g_{j}^{-}(v-\delta) \\
& \frac{\Gamma^{+}(u+\delta-v-a)}{\Gamma^{+}(u+\delta-v+a)}\left[x_{i}^{+}(u), x_{j}^{-}(v)\right] d u d v \\
= & -\hbar \delta_{i j} \oint_{\mathcal{C}_{i}^{+}} \oint_{\mathcal{C}_{i}^{-}} e^{2 \pi \iota k u} e^{2 \pi \iota \ell v} g_{i}^{+}(u+\delta) g_{i}^{-}(v-\delta) \\
& \frac{\Gamma^{+}\left(u+\delta-v-\hbar d_{i}\right)}{\Gamma^{+}\left(u+\delta-v+\hbar d_{i}\right)} \frac{\xi_{i}(u)-\xi_{i}(v)}{u-v} d u d v,
\end{aligned}
$$

where we used $(\mathcal{Y} 5)$.

Since the function $\Gamma^{+}\left(u+\delta-v-\hbar d_{i}\right)$ is holomorphic on $\bar{D}_{i}^{+} \times \bar{D}_{i}^{-}$for $|\delta|>r$, the only singularities of the integrand are the poles of $\xi_{i}(u), \xi_{i}(v)$. Choosing the contours $\mathcal{C}_{i}^{ \pm}$to be equal to a common contour $\mathcal{C}_{i}$ which contains all poles of $\xi_{i}$, Lemma 5.8 then yields

$$
I(\delta)=c_{i}^{+} c_{j}^{-} \hbar \delta_{i j} \oint_{\mathcal{C}_{i}} e^{2 \pi \iota(k+\ell) u} g_{i}^{+}(u+\delta) g_{i}^{-}(u-\delta) \frac{\Gamma^{+}\left(\delta-\hbar d_{i}\right)}{\Gamma^{+}\left(\delta+\hbar d_{i}\right)} \xi_{i}(u) d u .
$$

Since both $I(\delta)$ and the right-hand side $J(\delta)$ of (5.13) are holomorphic functions on the disk $|\delta|<R, I(0)$ is equal to

$$
\begin{aligned}
J(0) & =\delta_{i j} \hbar c_{i}^{+} c_{i}^{-} \frac{\Gamma\left(1-d_{i} \hbar\right)}{\Gamma\left(1+d_{i} \hbar\right)} \oint_{\mathcal{C}_{i}} e^{2 \pi \iota(k+\ell) u} g_{i}^{+}(u) g_{i}^{-}(u) \xi_{i}(u) d u \\
& =\delta_{i j} \frac{2 \pi \iota}{q_{i}-q_{i}^{-1}} \oint_{\mathcal{C}_{i}} e^{2 \pi \iota(k+\ell) u} S_{i}(u) d u \\
& =\frac{\delta_{i j}}{q_{i}-q_{i}^{-1}} \oint_{\widetilde{\mathcal{C}}_{i}} z^{k+\ell-1} \Psi_{i}(z) d z \\
& =\frac{\delta_{i j}}{q_{i}-q_{i}^{-1}}\left(\Psi_{i, k+\ell}^{+}-\Psi_{i, k+\ell}^{-}\right)
\end{aligned}
$$

where the second equality follows from (5.9), (5.5), and (5.8), the third from the change of variables $z=\exp (2 \pi \iota u) 6$ and the fourth from the fact that $\widetilde{\mathcal{C}}_{i}=$ $\exp \left(2 \pi \iota \mathcal{C}_{i}\right)$ encloses all the poles of $\Psi_{i}$ by 5.2 (i), and neither $z=0$ nor $z=\infty$.

5.10. Compatibility with shift automorphisms. Let $\rho: Y_{\hbar}(\mathfrak{g}) \rightarrow \operatorname{End}(V)$ be the action of $Y_{\hbar}(\mathfrak{g})$ on $V$ and $\rho_{a}: Y_{\hbar}(\mathfrak{g}) \rightarrow \operatorname{End}(V)$ the pull-back of $\rho$ via the shift automorphism $\tau_{a}$ of $Y_{\hbar}(\mathfrak{g})$. Thus, for $y=\xi_{i}, x_{i}^{ \pm}$we have

$$
\rho_{a}(y(u))=\rho(y(u-a)) .
$$

We need to show that for $Y=\Psi_{i}, \mathcal{X}_{i}^{ \pm}$, the following holds on $V$

$$
\Gamma\left(\rho_{a}\right)(Y(z))=\Gamma(\rho)\left(Y\left(e^{-2 \pi \iota a} z\right)\right) .
$$

For notational convenience, we denote the left-hand side by $\tilde{Y}(z)$ and the righthand side by $Y\left(e^{-2 \pi \iota a} z\right)$. By definition, $\widetilde{\Psi_{i}}(z)$ is obtained from the monodromy of the difference equation

$$
\widetilde{\phi}_{i}(u+1)=\xi_{i}(u-a) \widetilde{\phi}_{i}(u) .
$$

\footnotetext{
${ }^{6}$ We are assuming $\mathcal{C}_{i}$ to be small enough that the exponential map is injective on each of the connected components of its interior domain $D_{i}$.
} 
Clearly if $\phi_{i}^{ \pm}(u)$ and $S_{i}(u)$ are the fundamental solutions and the connection matrix of $\phi_{i}(u+1)=\xi_{i}(u) \phi_{i}(u)$, then $\phi_{i}^{ \pm}(u-a), S_{i}(u-a)$ are those of the difference equation $\widetilde{\phi}_{i}(u+1)=\xi_{i}(u-a) \widetilde{\phi}_{i}(u)$. Therefore, we get

$$
\widetilde{\Psi_{i}}(z)=\left.S_{i}(u-a)\right|_{z=e^{2 \pi \iota u}}=\left.S_{i}(u)\right|_{z=e^{2 \pi \iota(u+a)}}=\Psi_{i}\left(e^{-2 \pi \iota a} z\right) .
$$

Next, if $\mathcal{C}$ is the contour used to define $\mathcal{X}_{i}^{ \pm}(z)$, then $\mathcal{C}+a$ can be taken as the contour for $\widetilde{\mathcal{X}_{i}^{ \pm}}(z)$. It follows that

$$
\begin{aligned}
\widetilde{\mathcal{X}_{i}^{ \pm}}(z) & =c_{i}^{ \pm} \oint_{\mathcal{C}+a} \frac{z}{z-e^{2 \pi \iota u}} g_{i}^{ \pm}(u-a) x_{i}^{ \pm}(u-a) d u \\
& =c_{i}^{ \pm} \oint_{\mathcal{C}} \frac{z}{z-e^{2 \pi \iota(u+a)}} g_{i}^{ \pm}(u) x_{i}^{ \pm}(u) d u \\
& =\mathcal{X}_{i}^{ \pm}\left(e^{-2 \pi \iota a} z\right) .
\end{aligned}
$$

\section{THE INVERSE FUNCTOR 7}

Let $\Omega \subset \mathbb{C}^{\times}$be a subset stable under multiplication by $q$, and $\Pi \subset \mathbb{C}$ a noncongruent subset stable under shifts by $\frac{1}{2} \mathbb{Z} \hbar$ such that $\Omega=\exp (2 \pi \iota \Pi)$. In this section, we construct a functor

$$
\urcorner_{\Pi}: \mathcal{O}_{\text {int }}^{\Omega}\left(U_{q}(L \mathfrak{g})\right) \rightarrow \mathcal{O}_{\text {int }}^{\Pi}\left(Y_{\hbar}(\mathfrak{g})\right),
$$

which is an inverse to the functor $\Gamma_{\Pi}$ given by Theorem 5.4 .

6.1. Inverse monodromy problem. Let $\mathcal{V} \in \mathcal{O}_{\text {int }}^{\Omega}\left(U_{q}(L \mathfrak{g})\right)$, and let $\mu \in \mathfrak{h}^{*}$ be a weight of $\mathcal{V}$. For any $i \in \mathbf{I}$, Proposition 3.6 yields a $G L\left(\mathcal{V}_{\mu}\right)$-valued rational function $\Psi_{i}(z)_{\mu}$ satisfying

$$
\Psi_{i}(\infty)_{\mu}=q_{i}^{\mu\left(\alpha_{i}^{\vee}\right)}=\Psi_{i}(0)_{\mu}^{-1}
$$

and such that the poles of $\Psi_{i}(z)_{\mu}^{ \pm 1}$ are contained in $\Omega$. Set $A_{0}=\hbar d_{i} \mu\left(\alpha_{i}^{\vee}\right)$ so that $\Psi_{i}(\infty)_{\mu}=\exp \left(\pi \iota A_{0}\right)$, and $S_{i}(u)=\Psi\left(e^{2 \pi \iota u}\right)_{\mu}$.

Lemma. There exists a unique rational function $A(u): \mathbb{C} \rightarrow G L\left(\mathcal{V}_{\mu}\right)$ such that

- $A(u)=1+A_{0} u^{-1}+\cdots$.

- $[A(u), A(v)]=0$ for any $u, v$.

- the poles of $A(u)^{ \pm 1}$ are contained in $\Pi$.

- $S_{i}(u)$ is the connection matrix of $\phi(u+1)=A(u) \phi(u)$.

Proof. By Theorem 4.14, the inverse monodromy problem may be solved by making a choice of logarithms of the zeros and poles of the eigenvalues of $\Psi_{i}(z)_{\mu}$, and of the poles of the unipotent part of $\Psi_{i}(z)_{\mu}$. Choosing the logarithms to lie in $\Pi$ leads to a unique coefficient matrix $A(u)$ since $\Pi$ is non-congruent. We do, however, need to check that the choices are consistent in the sense of Equation (4.10).

Let $\gamma(z)$ be an eigenvalue of $\Psi_{i}(z)$ on the weight space $\mathcal{V}_{\mu}$. By [24] and 32, Proposition 16], there are two monic polynomials $Q(z), R(z)$, not vanishing at $z=0$, and such that

$$
\gamma(z)=q_{i}^{\operatorname{deg}(R)-\operatorname{deg}(Q)} \frac{Q\left(q_{i}^{2} z\right)}{Q(z)} \frac{R(z)}{R\left(q_{i}^{2} z\right)} .
$$

Let $Q(z)=\prod_{j}\left(z-\beta_{j}\right)$ and $R(z)=\prod_{k}\left(z-\beta_{k}^{\prime}\right)$. Let $b_{j}, b_{k}^{\prime} \in \Pi$ be such that $e^{2 \pi \iota b_{j}}=$ $\beta_{j}$ and $e^{2 \pi \iota b_{k}^{\prime}}=\beta_{k}^{\prime}$. Since $\Pi$ is stable under shifts by $\hbar / 2, b_{j}-d_{i} \hbar, b_{k}^{\prime}-d_{i} \hbar$ are 
logarithms of $q_{i}^{-2} \beta_{j}$ and $q_{i}^{-2} \beta_{k}^{\prime}$, respectively, which lie in $\Pi$. Since $A_{0}=\hbar d_{i} \mu\left(\alpha_{i}^{\vee}\right)$, the consistency requirement (4.10) reads

$$
\sum_{j} b_{j}+\sum_{k}\left(b_{k}^{\prime}-d_{i} \hbar\right)-\sum_{j}\left(b_{j}-d_{i} \hbar\right)-\sum_{k} b_{k}^{\prime}=\hbar d_{i} \mu\left(\alpha_{i}^{\vee}\right) .
$$

The latter holds since $\Psi_{i}(\infty)$ acts on $\mathcal{V}_{\mu}$ as multiplication by $q_{i}^{\mu\left(\alpha_{i}^{\vee}\right)}, \gamma(\infty)=$ $q_{i}^{\operatorname{deg} Q-\operatorname{deg} R}$, and $q$ is not a root of unity.

6.2. Yangian operators. Given $\mathcal{V} \in \mathcal{O}_{\text {int }}^{\Omega}\left(U_{q}(L \mathfrak{g})\right)$, and a weight $\mu \in \mathfrak{h}^{*}$ of $\mathcal{V}$, define the action of the generators of $Y_{\hbar}(\mathfrak{g})$ on $\mathcal{V}_{\mu}$ as follows.

(i) The action of $\mathfrak{h}$ on $\mathcal{V}_{\mu}$ is by weight $\mu$. Note that $K_{h}=q^{\mu(h)}$ on $\mathcal{V}_{\mu}$ for every $h \in \mathfrak{h}$ determines $\mu \in \mathfrak{h}^{*}$ uniquely.

(ii) $\xi_{i}(u)_{\mu}$ acts as the coefficient matrix $A(u)$ given by Lemma 6.1

Let $\phi_{i}^{ \pm}(u)_{\mu}$ be the fundamental solutions of the difference equation determined by $\xi_{i}(u)_{\mu}$, and define $g_{i}^{ \pm}(u)_{\mu}$ by (5.2). Let $\mathcal{C}_{i, \mu}^{ \pm} \subset \mathbb{C}$ be a Jordan curve such that

(a) $\mathcal{C}_{i, \mu}^{ \pm}$encloses all the poles of $\mathcal{X}_{i}^{ \pm}\left(e^{2 \pi \iota u}\right)$ contained in $\Pi$, and none of their $\mathbb{Z}_{\neq 0}$-translates.

(b) $\mathcal{C}_{i, \mu}^{ \pm}$encloses none of the $\mathbb{Z}_{\neq 0}$-translates of the poles of $\xi_{i}(u)_{\mu}^{-1}$.

Note that such a curve exists since the poles of $\xi_{i}(u)_{\mu}^{-1}$ are contained in $\Pi$, and the latter is non-congruent.

(iii) The generators $x_{i, r}^{ \pm}$act on $\mathcal{V}_{\mu}$ by

$$
\left(x_{i, r}^{ \pm}\right)_{\mu}=\frac{1}{c_{i}^{ \pm} \hbar} \int_{\mathcal{C}_{i, \mu}^{ \pm}} v^{r} g_{i}^{ \pm}(v)_{\mu \pm \alpha_{i}}^{-1} \mathcal{X}_{i}^{ \pm}\left(e^{2 \pi \iota v}\right)_{\mu} d v .
$$

Note that these operators are independent of the choice of the contour $\mathcal{C}_{i, \mu}^{ \pm}$since, by assumption (b), (5.3), and (5.4), $g_{i}^{ \pm}(v)_{\mu \pm \alpha_{i}}^{-1}$ are holomorphic inside $\mathcal{C}_{i, \mu}^{ \pm}$. In terms of the fields $x_{i}^{ \pm}(u)$, (6.1) may be rewritten as

$$
x_{i}^{ \pm}(u)_{\mu}=\frac{1}{c_{i}^{ \pm}} \int_{\mathcal{C}_{i, \mu}^{ \pm}} \frac{1}{u-v} g_{i}^{ \pm}(v)_{\mu \pm \alpha_{i}}^{-1} \mathcal{X}_{i}^{ \pm}\left(e^{2 \pi \iota v}\right)_{\mu} d v
$$

for $u$ not enclosed by $\mathcal{C}_{i, \mu}^{ \pm}$.

6.3 .

\section{Theorem.}

(i) The above operators define an action of $Y_{\hbar}(\mathfrak{g})$ on $\mathcal{V}$.

(ii) As a representation of $Y_{\hbar}(\mathfrak{g}), \mathcal{V}$ lies in $\mathcal{O}_{\text {int }}^{\Pi}\left(Y_{\hbar}(\mathfrak{g})\right)$.

(iii) The corresponding functor $\rceil_{\Pi}: \mathcal{O}_{\text {int }}^{\Omega}\left(U_{q}(L \mathfrak{g})\right) \rightarrow \mathcal{O}_{\text {int }}^{\Pi}\left(Y_{\hbar}(\mathfrak{g})\right)$ satisfies

$$
\left.\left.\Gamma_{\Pi} \circ\right\rceil_{\Pi}=\mathbf{1}_{\mathcal{O}_{\text {int }}^{\Omega}\left(U_{q}(L \mathfrak{g})\right)} \quad \text { and } \quad\right\rceil_{\Pi} \circ \Gamma_{\Pi}=\mathbf{1}_{\mathcal{O}_{\text {int }}^{\Pi}\left(Y_{\hbar}(\mathfrak{g})\right)} .
$$

In particular, if $\Pi$ is a fundamental domain for $u \mapsto u+1, \Gamma_{\Pi}$ gives rise to an isomorphism of categories $\mathcal{O}_{\text {int }}^{\Pi}\left(Y_{\hbar}(\mathfrak{g})\right) \stackrel{\sim}{\rightarrow} \mathcal{O}_{\text {int }}\left(U_{q}(L \mathfrak{g})\right)$.

(iv) The functor $\rceil_{\Pi}$ is compatible with shift automorphisms. That is, for any $\mathcal{V} \in \mathcal{O}_{\text {int }}^{\Omega}\left(U_{q}(L \mathfrak{g})\right)$, and $a \in \mathbb{C}$,

$$
\urcorner_{\Pi+a}\left(\mathcal{V}\left(e^{2 \pi \iota a}\right)\right)=\right\urcorner_{\Pi}(\mathcal{V})(a) .
$$


Proof. (i) The relations (Y0), (Y1), and (Y2) hold trivially. After some preparatory work in 6.5 and 6.6, we prove (Y3) in Section 6.7. (Y4) in Section 6.8, and (Y5) in Section 6.9. Relation (Y6) follows from Proposition 2.7

(ii) By construction, $\left\{\xi_{i}(u)\right\}_{i \in \mathbf{I}}$ have zeros and poles in $\Pi$. It follows by Theorem 3.8 part (ii) that $\urcorner_{\Pi}(\mathcal{V})$ lies in $\mathcal{O}_{\text {int }}^{\Pi}\left(Y_{\hbar}(\mathfrak{g})\right)$.

(iii) The fact that $\left.\Gamma_{\Pi} \circ\right\urcorner_{\Pi}(\mathcal{V})=\mathcal{V}$ as modules over the subalgebra of $U_{q}(L \mathfrak{g})$ generated by $\left\{\Psi_{i, \pm r}^{ \pm}\right\}_{i \in \mathbf{I}, r \in \mathbb{N}}$ follows from the definition of $\Gamma$, and the fact that Lemma 6.1 solves an inverse monodromy problem. The fact that $\rceil_{\Pi} \circ \Gamma_{\Pi}(V)=V$ as modules over the commutative subalgebra of $Y_{\hbar}(\mathfrak{g})$ generated by $\left\{\xi_{i, r}\right\}_{i \in \mathbf{I}, r \in \mathbb{N}}$ follows from the uniqueness statement of Lemma 6.1. The corresponding assertions for the generators $x_{i, r}^{ \pm}, \mathcal{X}_{i, k}^{ \pm}$of $Y_{\hbar}(\mathfrak{g}), U_{q}(L \mathfrak{g})$ are checked in 6.10 and 6.11 respectively.

(iv) Follows by a direct check similar to that in 5.10 or by applying $\Gamma_{\Pi+a}$ and using Theorem 5.4 (ii).

6.4 .

Remark. We observe that one can always find $\Pi \subset \mathbb{C}$ which is stable under shifts by $\frac{\hbar}{2}$ and is a fundamental domain for $u \mapsto u+1$, assuming that $\hbar$ is not rational. If $\operatorname{Im}(\hbar) \neq 0, \Pi$ may be taken to be the strip bounded between $\mathbb{R} \hbar$ and $\mathbb{R} \hbar+1$ including $\mathbb{R} \hbar$.

If $\hbar \in \mathbb{R} \backslash \mathbb{Q}$, we claim that there exists $U \subset \mathbb{R}$ such that $U \pm \frac{\hbar}{2} \subset U$ and $u \mapsto e^{2 \pi \iota u}$ is a bijection between $U$ and $S^{1}$. Assuming this, take $\Pi=\{u+\iota t: u \in U, t \in \mathbb{R}\}$. To prove the claim, we shall use Zorn's lemma as follows. Let $\mathcal{U}$ be the set of all subsets $V \subset \mathbb{R}$ such that $V$ is stable under shifts by $\hbar / 2$ and $u \mapsto e^{2 \pi \iota u}$ is an injective map from $V$ to $S^{1}$. The set $\mathcal{U}$ is non-empty, since $\mathbb{Z} \frac{\hbar}{2}$ is an element of $\mathcal{U}$. Moreover, every chain in $\mathcal{U}$ has the largest element in $\mathcal{U}$, and hence by Zorn's lemma $\mathcal{U}$ has a maximal element $U$. We claim that $u \mapsto e^{2 \pi \iota u}$ is a bijection between $U$ and $S^{1}$. If not, then there exists $x \in \mathbb{R}$ such that $x \not \equiv y(\bmod \mathbb{Z})$ for every $y \in U$. The same is true for every $x+n \hbar\left(n \in \frac{1}{2} \mathbb{Z}\right)$, and hence $U \cup\{x+n \hbar\}_{n \in(1 / 2) \mathbb{Z}}$ is also in $\mathcal{U}$, contradicting the maximality of $U$.

6.5 .

Lemma. Let $\mathcal{C}$ be a Jordan curve the interior domain $D$ of which is non-congruent, and $f$ a meromorphic function on a neighborhood of $\bar{D}$ with no singularities on $\mathcal{C}$. Let $a \in \mathbb{C} \backslash \mathbb{Z}$, and assume that

$$
\int_{\mathcal{C}} \frac{e^{2 \pi \iota(u+a)}-e^{2 \pi \iota v}}{e^{2 \pi \iota u}-e^{2 \pi \iota(v+a)}} f(v) d v=0
$$

for any $u \notin \bar{D}+a+\mathbb{Z}$. Then, $f$ has no poles in $D$.

Proof. Expanding the above integral near $e^{2 \pi \iota u}=\infty$ shows that

$$
\int_{\mathcal{C}} e^{2 \pi \iota k v} f(v) d v=0
$$

for any $k \in \mathbb{N}$, and therefore that $\int_{\mathcal{C}} p\left(e^{2 \pi \iota v}\right) f(v) d v=0$ for any polynomial $p$. Let us assume on the contrary that $\left\{b_{j}\right\}_{j \in J}$ are the poles of $f$ in $D$, and $\left\{n_{j}\right\}_{j \in J}$ their orders. For any $j \in J$, set

$$
p_{j}(w)=\left(w-\beta_{j}\right)^{n_{j}-1} \prod_{j^{\prime} \neq j}\left(w-\beta_{j^{\prime}}\right)^{n_{j^{\prime}}},
$$


where $\beta_{k}=e^{2 \pi \iota b_{k}}$. Then $p_{j}\left(e^{2 \pi \iota v}\right) f(v)$ is holomorphic on $D \backslash\left\{b_{j}\right\}$ and has a pole of order 1 at $v=b_{j}$ since $D$ is non-congruent. It follows that $\int_{\mathcal{C}} p_{j}\left(e^{2 \pi \iota v}\right) f(v) d v \neq 0$, a contradiction.

6.6. Let $i, j \in \mathbf{I}$, and set $a=\hbar d_{i} a_{i j} / 2$. The following is a converse to Proposition 5.5.

Proposition. Let $\mathcal{C}$ be a Jordan curve with interior domain $D$, and $f: \mathbb{C} \rightarrow$ $\operatorname{End}(\mathcal{V})$ a meromorphic function with no singularities in $\bar{D}$, and such that $\left[\Psi_{i}\left(e^{2 \pi \iota u}\right)\right.$, $f(v)]=0$ for any $u, v$. Then, the following holds for any $\epsilon \in\{ \pm\}$.

(i) If $u \notin \bar{D} \pm \epsilon a+\mathbb{Z}$,

$$
\begin{aligned}
\operatorname{Ad}\left(\Psi_{i}\left(e^{2 \pi \iota u}\right)\right)^{ \pm 1} \int_{\mathcal{C}} f(v) \mathcal{X}_{j}^{\epsilon}\left(e^{2 \pi \iota v}\right) d v & \\
= & \int_{\mathcal{C}}\left(\frac{e^{2 \pi \iota(u+\epsilon a)}-e^{2 \pi \iota v}}{e^{2 \pi \iota u}-e^{2 \pi \iota(v+\epsilon a)}}\right)^{ \pm 1} f(v) \mathcal{X}_{j}^{\epsilon}\left(e^{2 \pi \iota v}\right) d v .
\end{aligned}
$$

Assume moreover that $D$ is such that

(1) $D$ is non-congruent.

(2) $\bar{D}+a$ is non-congruent to $\bar{D}-a$.

(3) $\bar{D} \pm a$ are non-congruent to the set of poles of $\xi_{i}(u)^{ \pm 1}$.

Then, the following holds for any $\epsilon, \eta \in\{ \pm\}$.

(ii) If $u \notin \bar{D} \pm \epsilon a-\eta \mathbb{Z}_{>0}$,

$$
\operatorname{Ad}\left(g_{i}^{\eta}(u)\right)^{ \pm 1} \int_{\mathcal{C}} f(v) \mathcal{X}_{j}^{\epsilon}\left(e^{2 \pi \iota v}\right) d v=\int_{\mathcal{C}}\left(\frac{\Gamma^{\eta}(u-v-\epsilon a)}{\Gamma^{\eta}(u-v+\epsilon a)}\right)^{ \pm 1} f(v) \mathcal{X}_{j}^{\epsilon}\left(e^{2 \pi \iota v}\right) d v
$$

(iii) If $u \notin \bar{D} \pm \epsilon a$,

$$
\operatorname{Ad}\left(\xi_{i}(u)\right)^{ \pm 1} \int_{\mathcal{C}} f(v) \mathcal{X}_{j}^{\epsilon}\left(e^{2 \pi \iota v}\right) d v=\int_{\mathcal{C}}\left(\frac{u-v+\epsilon a}{u-v-\epsilon a}\right)^{ \pm 1} f(v) \mathcal{X}_{j}^{\epsilon}\left(e^{2 \pi \iota v}\right) d v
$$

Proof. (i) follows by left multiplying $(\mathcal{Q L} 3)$ by $f$ and integrating along $\mathcal{C}$.

(ii) and (iii) Fix a weight $\mu$ of $\mathcal{V}$, and let $E^{\epsilon} \subseteq \operatorname{Hom}\left(\mathcal{V}_{\mu}, \mathcal{V}_{\mu+\epsilon \alpha_{j}}\right)$ be the subspace spanned by elements of the form $\int_{\mathcal{C}} f(v) \mathcal{X}_{j}^{\epsilon}\left(e^{2 \pi \iota v}\right) d v$, where $f(v)$ is meromorphic with no singularities in $\bar{D}$, and commutes with $\Psi_{i}\left(e^{2 \pi \iota u}\right)$ for all $u, v$. By (i), $E^{\epsilon}$ is stable under $\operatorname{Ad}\left(\Psi_{i}\left(e^{2 \pi \iota u}\right)\right)$ for $u \notin \bar{D}+\epsilon a+\mathbb{Z}$, and therefore for any $u$ since $\Psi_{i}\left(e^{2 \pi \iota u}\right)$ is meromorphic in $u$. It follows that $E^{\epsilon}$ is stable under $\operatorname{Ad}\left(\xi_{i}(u)\right)$ and $\operatorname{Ad}\left(g_{i}^{\eta}(u)\right)$. We now wish to show that the action of $\operatorname{Ad}\left(g_{i}^{\eta}(u)\right)$ and $\operatorname{Ad}\left(\xi_{i}(u)\right)$ on $E^{\epsilon}$ is given by the formulas (ii) and (iii). We shall do so by showing that the formulas (ii) define meromorphic automorphisms of $E^{\epsilon}$ which factorize $\operatorname{Ad}\left(\Psi_{i}\left(e^{2 \pi \iota u}\right)\right)$, and then relying on the uniqueness statement of Proposition 4.11,

For $u \notin \bar{D}+\epsilon a-\eta \mathbb{Z}_{>0}$, define $\mathcal{G}^{\eta}(u) \in G L\left(E^{\epsilon}\right)$ by

$$
\int_{\mathcal{C}} f(v) \mathcal{X}_{j}^{\epsilon}\left(e^{2 \pi \iota v}\right) d v \longrightarrow \int_{\mathcal{C}} \frac{\Gamma^{\eta}(u-v-\epsilon a)}{\Gamma^{\eta}(u-v+\epsilon a)} f(v) \mathcal{X}_{j}^{\epsilon}\left(e^{2 \pi \iota v}\right) d v .
$$

Note that this is well-defined by (i), Lemma 6.5 and the fact that $D$ is noncongruent. Since the right-hand side is a meromorphic function of $u \in \mathbb{C}$, and lies in $E^{\epsilon}$ for $u \notin \bar{D}+\epsilon a-\eta \mathbb{Z}_{>0}$, it lies in $E^{\epsilon}$ for all $u$. 
It is clear that $\mathcal{G}^{\eta}(u)$ are invertible and holomorphic for $\operatorname{Re}( \pm u) \gg 0$, and that $\mathcal{G}^{+}(u)^{-1} \mathcal{G}^{-}(u)=\left.\operatorname{Ad}\left(\Psi_{i}\left(e^{2 \pi \iota u}\right)\right)\right|_{E^{\epsilon}}$. Set

$$
\Xi(u)=\mathcal{G}^{+}(u+1) \mathcal{G}^{+}(u)^{-1}=\mathcal{G}^{-}(u+1) \mathcal{G}^{-}(u)^{-1} .
$$

By inspection, $\Xi(u)^{ \pm 1}$ is given by

$$
\int_{\mathcal{C}} f(v) \mathcal{X}_{j}^{\epsilon}\left(e^{2 \pi \iota v}\right) d v \longrightarrow \int_{\mathcal{C}}\left(\frac{u-v+\epsilon a}{u-v-\epsilon a}\right)^{ \pm 1} f(v) \mathcal{X}_{j}^{\epsilon}\left(e^{2 \pi \iota v}\right) d v
$$

for any $u \notin \bar{D} \pm \epsilon a$. Since the poles of $\Xi(u)^{ \pm 1}$ are contained in $\bar{D} \pm \epsilon a$, and those of $\left.\operatorname{Ad}\left(\xi_{i}(u)\right)\right|_{E^{\epsilon}}$ are contained in $\Pi$ which is non-congruent, it follows from our assumptions on $D$ and Proposition 4.11 that $\Xi(u)=\left(\left.\operatorname{Ad}\left(\xi_{i}(u)\right)\right|_{E^{\epsilon}}\right.$, and therefore that $\mathcal{G}^{\eta}(u)=\left(\left.\operatorname{Ad}\left(g_{i}^{\eta}(u)\right)\right|_{E^{\epsilon}}\right.$.

6.7. Proof of (Y3). It suffices to prove $(\mathcal{Y} 3)$ for $u, v$ large enough. Let $i, j \in \mathbf{I}$, and set $a=\hbar d_{i} a_{i j} / 2$. Since the contour $\mathcal{C}_{j}^{ \pm}$defining $x_{j}^{ \pm}(v)$ in (6.1) is only required to contain the poles of $\xi_{j}(v)$, and the latter are contained in $\Pi$ which is noncongruent and stable under shift by $\hbar / 2$, we may choose $\mathcal{C}_{j}^{ \pm}$so that it also satisfies the conditions (1)-(3) of Proposition 6.6. It then follows that

$$
\xi_{i}(u) x_{j}^{ \pm}(v) \xi_{i}(u)^{-1}=\frac{1}{c_{j}^{ \pm}} \int_{\mathcal{C}_{j}^{ \pm}} \frac{u-v^{\prime} \pm a}{\left(v-v^{\prime}\right)\left(u-v^{\prime} \mp a\right)} g_{j}^{ \pm}\left(v^{\prime}\right)^{-1} \mathcal{X}_{j}^{ \pm}\left(e^{2 \pi \iota v^{\prime}}\right) d v^{\prime}
$$

so long as $u \mp a$ and $v$ do not lie within $\mathcal{C}_{j}$. (Y) 3$)$ now follows from the fact that

$$
\frac{u-v^{\prime} \pm a}{\left(v-v^{\prime}\right)\left(u-v^{\prime} \mp a\right)}=\frac{u-v \pm a}{u-v \mp a} \frac{1}{v-v^{\prime}} \mp \frac{2 a}{u-v \mp a} \frac{1}{u \mp a-v^{\prime}} \text {. }
$$

6.8. Proof of (Y4). We shall prove that

$$
x_{i, r+1}^{ \pm} x_{j, s}^{ \pm}-x_{i, r}^{ \pm} x_{j, s+1}^{ \pm} \mp a x_{i, r}^{ \pm} x_{j, s}^{ \pm}=x_{j, s}^{ \pm} x_{i, r+1}^{ \pm}-x_{j, s+1}^{ \pm} x_{i, r}^{ \pm} \pm a x_{j, s}^{ \pm} x_{i, r}^{ \pm} .
$$

The left-hand side, multiplied by $c_{i}^{ \pm} c_{j}^{ \pm}$, is equal to

$$
\frac{1}{\hbar^{2}} \int_{\mathcal{C}_{i}^{ \pm}} \int_{\mathcal{C}_{j}^{ \pm}} u^{r} v^{s}(u-v \mp a) g_{i}^{ \pm}(u)^{-1} \mathcal{X}_{i}^{ \pm}\left(e^{2 \pi \iota u}\right) g_{j}^{ \pm}(v)^{-1} \mathcal{X}_{j}^{ \pm}\left(e^{2 \pi \iota v}\right) d u d v .
$$

Choose $\mathcal{C}_{i}^{ \pm}, \mathcal{C}_{j}^{ \pm}$so that they satisfy conditions (1)-(3) of Proposition 6.6 with respect to $\xi_{j}(v)$ and $\xi_{i}(u)$, respectively, as well as

$$
\mathcal{C}_{j}^{ \pm} \cap\left(\bar{D}_{i} \pm a \mp \mathbb{Z}_{>0}\right)=\emptyset=\mathcal{C}_{i}^{ \pm} \cap\left(\bar{D}_{j} \pm a \mp \mathbb{Z}_{>0}\right),
$$

where $D_{i}, D_{j}$ are the interior domains of $\mathcal{C}_{i}^{ \pm}, \mathcal{C}_{j}^{ \pm}$. Then, the above is equal to

$$
\begin{aligned}
\frac{1}{\hbar^{2}} \int_{\mathcal{C}_{i}^{ \pm}} \int_{\mathcal{C}_{j}^{ \pm}} u^{r} v^{s}(u-v \mp a) \frac{\Gamma^{ \pm}(v-u \mp a)}{\Gamma^{ \pm}(v-u \pm a)} g_{i}^{ \pm}(u)^{-1} g_{j}^{ \pm}(v)^{-1} \\
\cdot \mathcal{X}_{i}^{ \pm}\left(e^{2 \pi \iota u}\right) \mathcal{X}_{j}^{ \pm}\left(e^{2 \pi \iota v}\right) d u d v .
\end{aligned}
$$

Similarly, the right-hand side of (6.3) multiplied by $c_{i}^{ \pm} c_{j}^{ \pm}$is equal to

$$
\begin{aligned}
\frac{1}{\hbar^{2}} \int_{\mathcal{C}_{i}^{ \pm}} \int_{\mathcal{C}_{j}^{ \pm}} u^{r} v^{s}(u-v \pm a) \frac{\Gamma^{ \pm}(u-v \mp a)}{\Gamma^{ \pm}(u-v \pm a)} g_{i}^{ \pm}(u)^{-1} g_{j}^{ \pm}(v)^{-1} \\
\cdot \mathcal{X}_{j}^{ \pm}\left(e^{2 \pi \iota v}\right) \mathcal{X}_{i}^{ \pm}\left(e^{2 \pi \iota u}\right) d u d v
\end{aligned}
$$


By $(\mathcal{Q L} 4)$, this may be rewritten as

$$
\begin{aligned}
\frac{1}{\hbar^{2}} \int_{\mathcal{C}_{i}^{ \pm}} \int_{\mathcal{C}_{j}^{ \pm}} u^{r} v^{s}(u-v \pm a) \frac{\Gamma^{ \pm}(u-v \mp a)}{\Gamma^{ \pm}(u-v \pm a)} \frac{e^{2 \pi \iota(v \pm a)}-e^{2 \pi \iota u}}{e^{2 \pi \iota v}-e^{2 \pi \iota(u \pm a)}} \\
\cdot g_{i}^{ \pm}(u)^{-1} g_{j}^{ \pm}(v)^{-1} \mathcal{X}_{i}^{ \pm}\left(e^{2 \pi \iota u}\right) \mathcal{X}_{j}^{ \pm}\left(e^{2 \pi \iota v}\right) d u d v
\end{aligned}
$$

Indeed, neither of the boundary terms $e^{2 \pi \iota v}\left(\mathcal{X}_{j, 0}^{ \pm} \mathcal{X}_{i}^{ \pm}\left(e^{2 \pi \iota u}\right)-q_{j}^{ \pm a_{j i}} \mathcal{X}_{i}^{ \pm}\left(e^{2 \pi \iota u}\right) \mathcal{X}_{j, 0}^{ \pm}\right)$ and $e^{2 \pi \iota u}\left(\mathcal{X}_{i, 0}^{ \pm} \mathcal{X}_{j}^{ \pm}\left(e^{2 \pi \iota v}\right)-q_{j}^{ \pm a_{j i}} \mathcal{X}_{j}^{ \pm}\left(e^{2 \pi \iota v}\right) \mathcal{X}_{i, 0}^{ \pm}\right)$from $(\mathcal{Q L} 4)$ contribute to the double integral since $(u-v \pm a) /\left(e^{2 \pi \iota v}-e^{2 \pi \iota(u \pm a)}\right)$ does not have any poles inside $\mathcal{C}_{i}^{ \pm} \times \mathcal{C}_{j}^{ \pm}$.

The result now follows from the fact that $\Gamma^{\mp}(x)=\Gamma^{ \pm}(-x)$, and (5.8).

6.9. Proof of (Y5). We need to prove that for any $i, j \in \mathbf{I}$ and $r, s \in \mathbb{N}$ we have

$$
\left[x_{i, r}^{+}, x_{j, s}^{-}\right]=\delta_{i j} \xi_{i, r+s} .
$$

Choosing the contours $\mathcal{C}_{i}^{+}, \mathcal{C}_{j}^{-}$as in 6.8, Proposition 6.6 yields

$$
\begin{aligned}
x_{i, r}^{+} x_{j, s}^{-}= & \frac{1}{c_{i}^{+} c_{j}^{-} \hbar^{2}} \int_{\mathcal{C}_{i}^{+}} \int_{\mathcal{C}_{j}^{-}} u^{r} v^{s} g_{i}^{+}(u)^{-1} \mathcal{X}_{i}^{+}\left(e^{2 \pi \iota u}\right) g_{j}^{-}(v)^{-1} \mathcal{X}_{j}^{-}\left(e^{2 \pi \iota v}\right) d u d v \\
= & \frac{1}{c_{i}^{+} c_{j}^{-} \hbar^{2}} \int_{\mathcal{C}_{i}^{+}} \int_{\mathcal{C}_{j}^{-}} u^{r} v^{s} g_{i}^{+}(u)^{-1} g_{j}^{-}(v)^{-1} \\
& \frac{\Gamma^{-}(v-u-a)}{\Gamma^{-}(v-u+a)} \mathcal{X}_{i}^{+}\left(e^{2 \pi \iota u}\right) \mathcal{X}_{j}^{-}\left(e^{2 \pi \iota v}\right) d u d v .
\end{aligned}
$$

Similarly,

$$
\begin{aligned}
x_{j, s}^{-} x_{i, r}^{+}= & \frac{1}{c_{i}^{+} c_{j}^{-} \hbar^{2}} \int_{\mathcal{C}_{i}^{+}} \int_{\mathcal{C}_{j}^{-}} u^{r} v^{s} g_{i}^{+}(u)^{-1} g_{j}^{-}(v)^{-1} \\
& \cdot \frac{\Gamma^{+}(u-v+a)}{\Gamma^{+}(u-v-a)} \mathcal{X}_{j}^{-}\left(e^{2 \pi \iota v}\right) \mathcal{X}_{i}^{+}\left(e^{2 \pi \iota u}\right) d u d v
\end{aligned}
$$

Since $\Gamma^{+}(x)=\Gamma^{-}(-x)$, it follows that

$$
\begin{aligned}
{\left[x_{i, r}^{+}, x_{j, s}^{-}\right]=\frac{1}{c_{i}^{+} c_{j}^{-} \hbar^{2}} \int_{\mathcal{C}_{i}^{+}} \int_{\mathcal{C}_{j}^{-}} u^{r} v^{s} g_{i}^{+}(u)^{-1} g_{j}^{-}(v)^{-1} } \\
\cdot \frac{\Gamma^{+}(u-v+a)}{\Gamma^{+}(u-v-a)}\left[\mathcal{X}_{i}^{+}\left(e^{2 \pi \iota u}\right), \mathcal{X}_{j}^{-}\left(e^{2 \pi \iota v}\right)\right] d u d v .
\end{aligned}
$$

By $(\mathcal{Q L} 5)$, we get

$$
\begin{aligned}
{\left[x_{i, r}^{+}, x_{j, s}^{-}\right]=\frac{\delta_{i j}}{q_{i}-q_{i}^{-1}} \frac{1}{c_{i}^{+} c_{j}^{-} \hbar^{2}} \int_{\mathcal{C}_{i}^{+}} \int_{\mathcal{C}_{j}^{-}} u^{r} v^{s} g_{i}^{+}(u)^{-1} g_{j}^{-}(v)^{-1} } \\
\cdot \frac{\Gamma^{+}(u-v+a)}{\Gamma^{+}(u-v-a)} \frac{\Psi_{i}\left(e^{2 \pi \iota v}\right)-e^{2 \pi \iota(v-u)} \Psi_{i}\left(e^{2 \pi \iota u}\right)}{1-e^{2 \pi \iota(v-u)}} d u d v
\end{aligned}
$$

since the contribution of the boundary term $\Psi_{i, 0}^{-}$to the double integral is zero given that the function $\Gamma^{+}(u-v+a)$ is holomorphic in $D_{i} \times D_{j}$. We may therefore assume that $i=j$. 
Note that

$$
\frac{\Psi_{i}\left(e^{2 \pi \iota v}\right)-e^{2 \pi \iota(v-u)} \Psi_{i}\left(e^{2 \pi \iota u}\right)}{1-e^{2 \pi \iota(v-u)}}=\frac{\Psi_{i}\left(e^{2 \pi \iota v}\right)-\Psi_{i}\left(e^{2 \pi \iota u}\right)}{1-e^{2 \pi \iota(v-u)}}+\Psi_{i}\left(e^{2 \pi \iota u}\right)
$$

and that the second summand on the right-hand side does not contribute to the double integral. Choosing the contours $\mathcal{C}_{i}^{ \pm}$to be equal to a common contour $\mathcal{C}_{i}$ which contains all poles of $\xi_{i}(u)$, we may apply Lemma 5.8 to find

$$
\left[x_{i, r}^{+}, x_{i, s}^{-}\right]=\frac{1}{q_{i}-q_{i}^{-1}} \frac{1}{c_{i}^{+} c_{i}^{-} \hbar^{2}} \frac{\Gamma^{+}\left(\hbar d_{i}\right)}{\Gamma^{-}\left(\hbar d_{i}\right)} \int_{\mathcal{C}_{i}} u^{r+s} g_{i}^{+}(u)^{-1} g_{i}^{-}(u)^{-1} \Psi_{i}\left(e^{2 \pi \iota u}\right) d u,
$$

where we used the fact that

$$
\lim _{u \rightarrow v} \frac{u-v}{1-e^{2 \pi \iota(v-u)}}=\frac{1}{2 \pi \iota} .
$$

Using (5.9), (5.5), and (4.9), we then get

$$
\left[x_{i, r}^{+}, x_{i, s}^{-}\right]=\frac{1}{2 \pi \iota \hbar} \int_{\mathcal{C}_{i}} u^{r+s} \xi_{i}(u) d u=\xi_{i, r+s} .
$$

6.10. Proof that $\rceil_{\Pi} \circ \Gamma_{\Pi}=1$. Let $V \in \mathcal{O}_{\text {int }}^{\Pi}\left(Y_{\hbar}(\mathfrak{g})\right)$. By (5.7), $x_{i}^{ \pm}(u)$ acts on \urcorner$_{\Pi} \circ \Gamma_{\Pi}(V)$ as

$$
\begin{aligned}
\frac{1}{c_{i}^{ \pm}} \int_{\mathcal{C}_{i}^{ \pm}} \frac{1}{u-v} g_{i}^{ \pm}(v)^{-1} \mathcal{X}_{i}^{ \pm}(v) d v & \\
& =\int_{\mathcal{C}_{i}^{ \pm}} \frac{1}{u-v} g_{i}^{ \pm}(v)^{-1}\left(\oint_{\mathcal{C}^{\prime}{ }_{i}^{ \pm}} \frac{1}{1-e^{-2 \pi \iota\left(v-v^{\prime}\right)}} g_{i}^{ \pm}\left(v^{\prime}\right) x_{i}^{ \pm}\left(v^{\prime}\right) d v^{\prime}\right) d v,
\end{aligned}
$$

where $u$ lies outside of $\mathcal{C}_{i}^{ \pm}$, and $\mathcal{C}_{i}^{ \pm}$lies outside of $\mathcal{C}^{\prime \pm}+\mathbb{Z}$. Assuming that $\mathcal{C}_{i}^{ \pm}$ contains $\mathcal{C}_{i}^{\prime \pm}$ and none of its $\mathbb{Z}_{\neq 0}$-translates and integrating in $v$ first yields

$$
\frac{1}{2 \pi \iota} \int_{\mathcal{C}^{\prime}{ }_{i}^{ \pm}} \frac{1}{u-v^{\prime}} x_{i}^{ \pm}\left(v^{\prime}\right) d v^{\prime}=x_{i}^{ \pm}(u)
$$

where the equality follows from the fact that $\mathcal{C}_{i}^{\prime}$ contains all the poles of $x_{i}^{ \pm}(u)$.

6.11. Proof that $\left.\Gamma_{\Pi} \circ\right\urcorner_{\Pi}=1$. Let $\mathcal{V} \in \mathcal{O}_{\text {int }}^{\Omega}\left(U_{q}(L \mathfrak{g})\right)$. Then, $\mathcal{X}_{i}^{ \pm}(z)$ acts on $\left.\Gamma_{\Pi} \circ\right\urcorner_{\Pi}(\mathcal{V})$ as

$$
\begin{aligned}
c_{i}^{ \pm} \oint_{\mathcal{C}_{i}^{ \pm}} \frac{z}{z-e^{2 \pi \iota v}} g_{i}^{ \pm}(v) x_{i}^{ \pm}(v) d v \\
\quad=\oint_{\mathcal{C}_{i}^{ \pm}} \frac{z}{z-e^{2 \pi \iota v}} g_{i}^{ \pm}(v) \int_{\mathcal{C}^{\prime}{ }_{i}^{ \pm}} \frac{1}{v-v^{\prime}} g_{i}^{ \pm}\left(v^{\prime}\right)^{-1} \mathcal{X}_{i}^{ \pm}\left(e^{2 \pi \iota v^{\prime}}\right) d v^{\prime} d v
\end{aligned}
$$

where $z$ lies outside of $\exp \left(2 \pi \iota \mathcal{C}_{i}^{ \pm}\right)$, and $\mathcal{C}_{i}^{ \pm}$lies outside of $\mathcal{C}_{i}^{\prime \pm}$. Assuming that $\mathcal{C}_{i}^{ \pm}$ contains $\mathcal{C}_{i}^{\prime}$, and integrating in $v$ first yields

$$
\int_{\mathcal{C}^{\prime}{ }_{i}^{ \pm}} \frac{z}{z-e^{2 \pi \iota v^{\prime}}} \mathcal{X}_{i}^{ \pm}\left(e^{2 \pi \iota v^{\prime}}\right) d v^{\prime}=\oint_{\widetilde{\mathcal{C}}^{\prime}{ }_{i}^{ \pm}} \frac{z}{z-w} \mathcal{X}_{i}^{ \pm}(w) \frac{d w}{w}
$$

where $\widetilde{\mathcal{C}}^{\prime}{ }_{i}^{ \pm}=\exp \left(2 \pi \iota \mathcal{C}_{i}^{\prime}{ }_{i}\right)$ contains all the poles of $\mathcal{X}_{i}^{ \pm}$. Since the one-form $d w /(z-w) w$ is regular on $\mathbb{P}^{1} \backslash\{z, 0\}$, and $\mathcal{X}_{i}^{ \pm}(0)=0$, the above is equal to $\mathcal{X}_{i}^{ \pm}(z)$. 


\section{7. q-Characters}

7.1. $q$-characters for $U_{q}(L \mathfrak{g})$. The $q$-characters of finite-dimensional representations of the quantum loop algebra $U_{q}(L \mathfrak{g})$ of a semisimple Lie algebra were introduced by Frenkel-Reshetikhin in [24, and later extended to the case of a symmetrizable Kac-Moody algebra by Hernandez in [31,32. We follow the presentation in [31, 32.

Let $\mathcal{V} \in \mathcal{O}_{\text {int }}\left(U_{q}(L \mathfrak{g})\right)$. Given a collection $\gamma=\left(\gamma_{i, \pm m}^{ \pm}\right)_{i \in \mathbf{I}, m \in \mathbb{N}}$ of complex numbers, and $\mu \in \mathfrak{h}^{*}$ such that $q_{i}^{ \pm \mu\left(\alpha_{i}^{\vee}\right)}=\gamma_{i, 0}^{ \pm}$, define $\mathcal{V}[\mu, \gamma]$ as follows

$$
\begin{aligned}
\mathcal{V}[\mu, \gamma]=\left\{v \in \mathcal{V} \mid K_{h} v\right. & =q^{\mu(h)} v, \text { and } \\
& \left.\exists p>0 \text { such that }\left(\Psi_{i, \pm m}^{ \pm}-\gamma_{i, \pm m}^{ \pm}\right)^{p} v=0 \forall i \in \mathbf{I}, m \in \mathbb{N}\right\} .
\end{aligned}
$$

Proposition. For a given pair $(\mu, \gamma)$, set

$$
\gamma_{i}(z)^{ \pm}=\sum_{m \geq 0} \gamma_{i, \pm m}^{ \pm} z^{\mp m} .
$$

Then, there exists $\mathcal{V} \in \mathcal{O}_{\text {int }}\left(U_{q}(L \mathfrak{g})\right)$ such that $\mathcal{V}[\mu, \gamma] \neq 0$ if and only if

(i) There exist monic polynomials $\left\{\mathcal{Q}_{i}(w), \mathcal{R}_{i}(w)\right\}$ such that $\mathcal{Q}_{i}(0) \neq 0$, $\mathcal{R}_{i}(0) \neq 0$, and for every $i \in \mathbf{I}$ we have

$$
\gamma_{i}(z)^{ \pm}=q_{i}^{-\operatorname{deg}\left(\mathcal{Q}_{i}\right)+\operatorname{deg}\left(\mathcal{R}_{i}\right)} \frac{\mathcal{Q}_{i}\left(q_{i}^{2} z\right)}{\mathcal{Q}_{i}(z)} \frac{\mathcal{R}_{i}(z)}{\mathcal{R}_{i}\left(q_{i}^{2} z\right)} .
$$

(ii) $\mu \leq \lambda$ for some $\lambda \in P_{+}$.

7.2. Consider the collection of variables $\left\{Y_{i, a}\right\}_{i \in \mathbf{I}, a \in \mathbb{C}^{\times}}$, and let $\mathcal{A}$ be the abelian group consisting of elements $e(\mu) \cdot M$, where $\mu \in \mathfrak{h}^{*}$ and $M$ is a Laurent monomial in the variables $\left\{Y_{i, a}\right\}$ such that

$$
\mu\left(\alpha_{i}^{\vee}\right)=\sum_{a \in \mathbb{C}^{\times}} \text {degree of } Y_{i, a} \text { in } M .
$$

The group operation on $\mathcal{A}$ is $e(\mu) \cdot M e\left(\mu^{\prime}\right) \cdot M^{\prime}=e\left(\mu+\mu^{\prime}\right) \cdot M M^{\prime}$, with $e(0) \cdot 1$ being the unit element.

Let $\mathcal{Y}$ be the group algebra of $\mathcal{A}$ over $\mathbb{Z}$, completed in the following sense. An element of $\mathcal{Y}$ is a formal (possibly infinite) linear combination $\chi$ of elements of $\mathcal{A}$ with $\mathbb{Z}$ coefficients, satisfying the following condition: there exist $\lambda_{1}, \ldots, \lambda_{r} \in P_{+}$ such that if $e(\mu) \cdot M$ appears in $\chi$ with a non-zero coefficient, then $\mu \leq \lambda_{j}$ for some $j \in\{1, \ldots, r\}$.

7.3. To each $(\mu, \gamma)$ of the form given by Proposition [7.1, we associate a monomial $M(\mu, \gamma) \in \mathcal{Y}$ as follows. Let $\left\{\mathcal{Q}_{i, \gamma}, \mathcal{R}_{i, \gamma}\right\}$ be a collection of polynomials associated to $\gamma$ so that (7.1) holds. If

$$
\mathcal{Q}_{i, \gamma}=\prod_{j}\left(w-a_{j}^{(i)}\right) \quad \text { and } \quad \mathcal{R}_{i, \gamma}=\prod_{k}\left(w-b_{k}^{(i)}\right),
$$

set

$$
M(\mu, \gamma)=e(\mu) \cdot \prod_{i \in \mathbf{I}}\left(\prod_{j} Y_{i, a_{j}^{(i)}}\right)\left(\prod_{k} Y_{i, b_{k}^{(i)}}\right)^{-1}
$$


The $q$-character of $\mathcal{V} \in \mathcal{O}_{\text {int }}\left(U_{q}(L \mathfrak{g})\right)$ is defined by

$$
\chi_{q}^{U}(\mathcal{V})=\sum_{(\mu, \gamma)} \operatorname{dim}(\mathcal{V}[\mu, \gamma]) M(\mu, \gamma) \in \mathcal{Y} .
$$

It gives rise to a homomorphism of abelian groups

$$
\chi_{q}^{U}: K\left(\mathcal{O}_{\text {int }}\left(U_{q}(L \mathfrak{g})\right)\right) \rightarrow \mathcal{Y} .
$$

Remark. When $\mathfrak{g}$ is a simple Lie algebra, the tensor structure on $\mathcal{O}_{\text {int }}\left(U_{q}(L \mathfrak{g})\right)$ induces a ring structure on $K\left(\mathcal{O}_{\text {int }}\left(U_{q}(L \mathfrak{g})\right)\right)$. In this case, it is proved in 24 that $\chi_{q}^{U}$ is an injective ring homomorphism.

7.4. $q$-characters for $Y_{\hbar}(\mathfrak{g})$. $q$-characters for the Yangian $Y_{\hbar}(\mathfrak{g})$ of a semisimple Lie algebra were introduced by Knight in [37. The treatment in [31,32] readily carries over to the Yangian and allows them to extend their definition to the case of a symmetrizable Kac-Moody algebra.

Given a collection $\beta=\left(\beta_{i, r}\right)_{i \in \mathbf{I}, r \in \mathbb{N}}$ of complex numbers, $\mu \in \mathfrak{h}^{*}$ such that $d_{i} \mu\left(\alpha_{i}^{\vee}\right)=\beta_{i, 0}$, and $V \in \mathcal{O}_{\text {int }}\left(Y_{\hbar}(\mathfrak{g})\right)$, define

$$
\begin{aligned}
V[\mu, \beta]=\{v \in V: h . v=\mu(h) v & \text { and } \\
& \left.\quad \exists p>0 \text { such that }\left(\xi_{i, r}-\beta_{i, r}\right)^{p} v=0 \forall i \in \mathbf{I}, r \in \mathbb{N}\right\} .
\end{aligned}
$$

Theorem. 37] Given a pair $(\mu, \beta)$ as above, set

$$
\beta_{i}(u)=1+\hbar \sum_{r \geq 0} \beta_{i, r} u^{-r-1} \in 1+u^{-1} \mathbb{C}\left[\left[u^{-1}\right]\right] .
$$

Then, there exists $V \in \mathcal{O}_{\text {int }}\left(Y_{\hbar}(\mathfrak{g})\right)$ such that $V[\mu, \beta] \neq 0$ if and only if

(i) There exist monic polynomials $\left\{Q_{i}, R_{i}\right\}_{i \in \mathbf{I}}$ such that for every $i \in \mathbf{I}$ we have

$$
\beta_{i}(u)=\frac{Q_{i}\left(u+\hbar d_{i}\right)}{Q_{i}(u)} \frac{R_{i}(u)}{R_{i}\left(u+\hbar d_{i}\right)} .
$$

(ii) $\mu \leq \lambda$ for some $\lambda \in P_{+}$.

7.5. Define a commutative ring $\mathcal{X}$ analogous to $\mathcal{Y}$, over the collection of variables

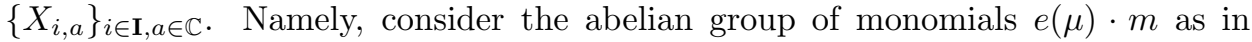
Section 7.2, except that $m$ is now a Laurent monomial in $\left\{X_{i, a}\right\}$. Then, $\mathcal{X}$ is the group algebra of this abelian group over $\mathbb{Z}$, completed in the same way as in Section 7.2 .

7.6. To a pair $(\mu, \beta)$ of the form given by Theorem 7.4, we associate a monomial $m(\mu, \beta) \in \mathcal{X}$ as follows. Let $\left\{Q_{i}, R_{i}\right\}$ be a set of monic polynomials associated to $\beta$ so that (7.3) holds. If

$$
Q_{i}=\prod_{j}\left(u-a_{j}^{(i)}\right) \quad \text { and } \quad R_{i}=\prod_{k}\left(u-b_{k}^{(i)}\right),
$$

set

$$
m(\mu, \beta)=e(\mu) \cdot \prod_{i \in \mathbf{I}}\left(\prod_{j} X_{i, a_{j}^{(i)}}\right)\left(\prod_{k} X_{i, b_{k}^{(i)}}\right)^{-1}
$$


The $q$-character of $V \in \mathcal{O}_{\text {int }}\left(Y_{\hbar}(\mathfrak{g})\right)$ is now defined as

$$
\chi_{q}^{Y}(V):=\sum_{(\mu, \beta)} \operatorname{dim}(V[\mu, \beta]) m(\mu, \beta) \in \mathcal{X}
$$

and gives rise to a homomorphism of abelian groups

$$
\chi_{q}^{Y}: K\left(\mathcal{O}_{\mathrm{int}}\left(Y_{\hbar}(\mathfrak{g})\right)\right) \rightarrow \mathcal{X} .
$$

For a semisimple Lie algebra $\mathfrak{g}, \chi_{q}^{Y}$ is an injective ring homomorphism [37].

7.7. Let $\mathcal{X}_{\Pi} \subset \mathcal{X}$ be the subring generated by $\mathfrak{h}^{*}$ and the variables $\left\{X_{i, a}\right\}_{i \in \mathbf{I}, a \in \Pi}$. By Theorem 3.8, $\chi_{q}^{Y}$ restricts to a homomorphism

$$
\chi_{q}^{Y}: K\left(\mathcal{O}_{\text {int }}^{\Pi}\left(Y_{\hbar}(\mathfrak{g})\right)\right) \rightarrow \mathcal{X}_{\Pi} .
$$

Similarly, let $\mathcal{Y}_{\Omega} \subset \mathcal{Y}$ be the subring generated by $\mathfrak{h}^{*}$ and $\left\{Y_{i, \alpha}\right\}_{i \in \mathbf{I}, \alpha \in \Omega}$. Again by Theorem 3.8, $\chi_{q}^{U}$ restricts to a homomorphism

$$
\chi_{q}^{U}: K\left(\mathcal{O}_{\text {int }}^{\Omega}\left(U_{q}(L \mathfrak{g})\right)\right) \rightarrow \mathcal{Y}_{\Omega} .
$$

Consider the isomorphism $e_{\Pi}: \mathcal{X}_{\Pi} \rightarrow \mathcal{Y}_{\Omega}$ which is identity on $\mathfrak{h}^{*}$ and maps $X_{i, a}$ to $Y_{i, e^{2 \pi \iota a}}$. The following result follows from the definition of the functor $\Gamma$ and the computation in 4.9 .

Proposition. The following diagram is commutative:

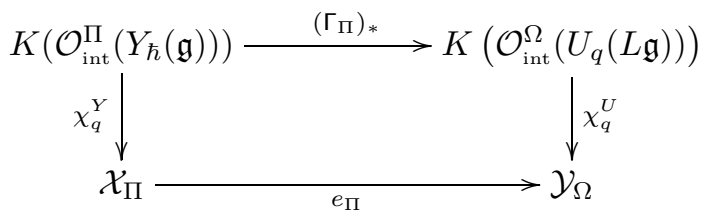

In particular, for a simple Lie algebra $\mathfrak{g},\left(\Gamma_{\Pi}\right)_{*}$ is an isomorphism of commutative rings.

\section{ACKNOWLEDGMENTS}

The authors are very grateful to Andrei Okounkov and Igor Krichever for many stimulating discussions, to David Hernandez for asking about the compatibility between the authors' construction and q-characters, and to Edward Frenkel for his numerous comments on an earlier version of this paper. Part of this project was completed while the authors were visiting ETH Zürich, while the second author was on a sabbatical leave at Columbia University, and during a month-long visit at the Aspen Center for Physics. The authors are grateful to these institutions for their support and wonderful working conditions, and to D. Calaque, G. Felder, A. Okounkov, D. Freed, G. Moore, A. Neitzke, and H. Ooguri for their invitations.

\section{REFERENCES}

[1] R. J. Baxter, Exactly solved models in statistical mechanics, Academic Press, Inc. [Harcourt Brace Jovanovich Publishers], London, 1982. MR690578 (86i:82002a)

[2] V. V. Bazhanov, Trigonometric solutions of triangle equations and classical Lie algebras, Phys. Lett. B 159 (1985), no. 4-6, 321-324, DOI 10.1016/0370-2693(85)90259-X. MR806529 (87g:82009) 
[3] V. V. Bazhanov, Integrable quantum systems and classical Lie algebras, Comm. Math. Phys. 113 (1987), no. 3, 471-503. MR925925 (89d:58051)

[4] V. V. Bazhanov, R. Frassek, T. Łukowski, C. Meneghelli, and M. Staudacher, Baxter Qoperators and representations of Yangians, Nuclear Phys. B 850 (2011), no. 1, 148-174, DOI 10.1016/j.nuclphysb.2011.04.006. MR2803592

[5] J. Beck and V. G. Kac, Finite-dimensional representations of quantum affine algebras at roots of unity, J. Amer. Math. Soc. 9 (1996), no. 2, 391-423, DOI 10.1090/S0894-0347-96-00183-X. MR 1317228 (96f:17015)

[6] G. D. Birkhoff, General theory of linear difference equations, Trans. Amer. Math. Soc. 12 (1911), no. 2, 243-284, DOI 10.2307/1988577. MR1500888

[7] G. D. Birkhoff, The generalized Riemann problem for linear differential equations and allied problems for linear difference and q-difference equations, Proc. Amer. Acad. Arts Sci. 49 (1913), 521-568.

[8] A. Borodin, Isomonodromy transformations of linear systems of difference equations, Ann. of Math. (2) 160 (2004), no. 3, 1141-1182, DOI 10.4007/annals.2004.160.1141. MR2144976 (2007b:37149)

[9] V. Chari and A. Pressley, Yangians and R-matrices, Enseign. Math. (2) 36 (1990), no. 3-4, 267-302. MR 1096420 (92h:17009)

[10] V. Chari and A. Pressley, Quantum affine algebras, Comm. Math. Phys. 142 (1991), no. 2, 261-283. MR 1137064 (93d:17017)

[11] V. Chari and A. Pressley, A guide to quantum groups, Cambridge University Press, Cambridge, UK, 1994.

[12] V. Chari and A. Pressley, Quantum affine algebras and their representations, Representations of groups (Banff, AB, 1994), CMS Conf. Proc., vol. 16, Amer. Math. Soc., Providence, RI, 1995, pp. 59-78. MR.1357195 (96j:17009)

[13] I. Cherednik, Double affine Hecke algebras, London Mathematical Society Lecture Note Series, vol. 319, Cambridge University Press, Cambridge, 2005. MR2133033 (2007e:32012)

[14] V. G. Drinfel'd, Hopf algebras and the quantum Yang-Baxter equation (Russian), Dokl. Akad. Nauk 283 (1985), no. 5, 1060-1064. MR802128 (87h:58080)

[15] V. G. Drinfel'd, Quantum groups, Proceedings of the International Congress of Mathematicians, Vol. 1, 2 (Berkeley, Calif., 1986), Amer. Math. Soc., Providence, RI, 1987, pp. 798-820. MR.934283 (89f:17017)

[16] V. G. Drinfel'd, A new realization of Yangians and of quantum affine algebras (Russian), Dokl. Akad. Nauk 296 (1987), no. 1, 13-17; English transl., Soviet Math. Dokl. 36 (1988), no. 2, 212-216. MR914215 (88j:17020)

[17] N. Dunford and J. T. Schwartz, Linear operators. Part I. General theory, John Wiley and Sons, New York, 1958.

[18] P. Etingof and O. Schiffmann, On highest weight modules over elliptic quantum groups, Lett. Math. Phys. 47 (1999), no. 2, 179-188, DOI 10.1023/A:1007543104904. MR.1682305 (2001f:17020)

[19] P. I. Etingof and A. A. Moura, On the quantum Kazhdan-Lusztig functor, Math. Res. Lett. 9 (2002), no. 4, 449-463, DOI 10.4310/MRL.2002.v9.n4.a5. MR1928865 (2003g:17022)

[20] L. Faddeev, Integrable models in $(1+1)$-dimensional quantum field theory, Recent advances in field theory and statistical mechanics (Les Houches, 1982), North-Holland, Amsterdam, 1984, pp. 561-608. MR782509 (87b:81002)

[21] B. Feigin, E. Feigin, M. Jimbo, T. Miwa, and E. Mukhin, Quantum continuous $\mathfrak{g l}_{\infty}$ : semiinfinite construction of representations, Kyoto J. Math. 51 (2011), no. 2, 337-364, DOI 10.1215/21562261-1214375. MR2793271 (2012k:17024)

[22] G. Felder, Conformal field theory and integrable systems associated to elliptic curves, Proceedings of the International Congress of Mathematicians, Vol. 1, 2 (Zürich, 1994), Birkhäuser, Basel, 1995, pp. 1247-1255. MR1404026 (97m:81015)

[23] E. Frenkel and D. Hernandez, Baxter's relations and spectra of quantum integrable models, Duke Math. J. 164 (2015), no. 12, 2407-2460, DOI 10.1215/00127094-3146282. MR3397389

[24] E. Frenkel and N. Reshetikhin, The q-characters of representations of quantum affine algebras and deformations of $\mathcal{W}$-algebras, Recent developments in quantum affine algebras and related topics (Raleigh, NC, 1998), Contemp. Math., vol. 248, Amer. Math. Soc., Providence, RI, 1999, pp. 163-205, DOI 10.1090/conm/248/03823. MR1745260 (2002f:17022) 
[25] I. B. Frenkel and N. Yu. Reshetikhin, Quantum affine algebras and holonomic difference equations, Comm. Math. Phys. 146 (1992), no. 1, 1-60. MR1163666 (94c:17024)

[26] S. Gautam and V. Toledano Laredo, Yangians and quantum loop algebras, Selecta Math. (N.S.) 19 (2013), no. 2, 271-336, DOI 10.1007/s00029-012-0114-2. MR.3090231

[27] S. Gautam and V. T. Laredo, Meromorphic Kazhdan-Lusztig equivalence for Yangians and quantum loop algebras, available at arXiv:1403.5251.

[28] S. Gautam and V. T. Laredo, Elliptic quantum groups and their finite-dimensional representations. In preparation.

[29] V. Ginzburg, Geometric methods in the representation theory of Hecke algebras and quantum groups, Representation theories and algebraic geometry (Montreal, PQ, 1997), NATO Adv. Sci. Inst. Ser. C Math. Phys. Sci., vol. 514, Kluwer Acad. Publ., Dordrecht, 1998, pp. 127-183. Notes by Vladimir Baranovsky [V. Yu. Baranovski1]. MR 1649626 (99j:17020)

[30] N. Guay and X. Ma, From quantum loop algebras to Yangians, J. Lond. Math. Soc. (2) 86 (2012), no. 3, 683-700, DOI 10.1112/jlms/jds021. MR3000826

[31] D. Hernandez, Algebraic approach to q,t-characters, Adv. Math. 187 (2004), no. 1, 1-52, DOI 10.1016/j.aim.2003.07.016. MR2074171(2005d:17020)

[32] D. Hernandez, Representations of quantum affinizations and fusion product, Transform. Groups 10 (2005), no. 2, 163-200, DOI 10.1007/s00031-005-1005-9. MR2195598 (2006k:17025)

[33] D. Hernandez, Drinfeld coproduct, quantum fusion tensor category and applications, Proc. Lond. Math. Soc. (3) 95 (2007), no. 3, 567-608, DOI 10.1112/plms/pdm017. MR2368277 (2008k:17017)

[34] H. Iritani, An integral structure in quantum cohomology and mirror symmetry for toric orbifolds, Adv. Math. 222 (2009), no. 3, 1016-1079, DOI 10.1016/j.aim.2009.05.016. MR.2553377 (2010j:53182)

[35] M. Jimbo, H. Konno, S. Odake, and J. Shiraishi, Quasi-Hopf twistors for elliptic quantum groups, Transform. Groups 4 (1999), no. 4, 303-327, DOI 10.1007/BF01238562. MR.1726695 (2002c:17026)

[36] V. G. Kac, Infinite-dimensional Lie algebras, 3rd ed., Cambridge University Press, Cambridge, 1990. MR1104219 (92k:17038)

[37] H. Knight, Spectra of tensor products of finite-dimensional representations of Yangians, J. Algebra 174 (1995), no. 1, 187-196, DOI 10.1006/jabr.1995.1123. MR.1332866 (96g:17015)

[38] I. M. Krichever, Analytic theory of difference equations with rational and elliptic coefficients and the Riemann-Hilbert problem (Russian, with Russian summary), Uspekhi Mat. Nauk 59 (2004), no. 6(360), 111-150, DOI 10.1070/RM2004v059n06ABEH000798; English transl., Russian Math. Surveys 59 (2004), no. 6, 1117-1154. MR2138470 (2005m:39036)

[39] S. Z. Levendorskiı̌, On generators and defining relations of Yangians, J. Geom. Phys. 12 (1993), no. 1, 1-11, DOI 10.1016/0393-0440(93)90084-R. MR1226802 (94d:17017)

[40] G. Lusztig, Affine Hecke algebras and their graded version, J. Amer. Math. Soc. 2 (1989), no. 3, 599-635, DOI 10.2307/1990945. MR991016 (90e:16049)

[41] G. Lusztig, Introduction to quantum groups, Progress in Mathematics, vol. 110, Birkhäuser Boston, Inc., Boston, MA, 1993. MR1227098 (94m:17016)

[42] D. Maulik and A. Okounkov, Quantum Groups and Quantum Cohomology, available at arXiv:1211.1287.

[43] K. Miki, $A(q, \gamma)$ analog of the $W_{1+\infty}$ algebra, J. Math. Phys. 48 (2007), no. 12, 123520, 35, DOI 10.1063/1.2823979. MR2377852(2009a:17024)

[44] H. Nakajima, Quiver varieties and finite-dimensional representations of quantum affine algebras, J. Amer. Math. Soc. 14 (2001), no. 1, 145-238, DOI 10.1090/S0894-0347-00-00353-2. MR 1808477 (2002i:17023)

[45] A. Okounkov and R. Pandharipande, The quantum differential equation of the Hilbert scheme of points in the plane, Transform. Groups 15 (2010), no. 4, 965-982, DOI 10.1007/s00031010-9116-3. MR2753265(2012h:14142)

[46] O. Schiffmann and E. Vasserot, The elliptic Hall algebra, Cherednik Hecke algebras and Macdonald polynomials, Compos. Math. 147 (2011), no. 1, 188-234, DOI 10.1112/S0010437X10004872. MR.2771130 (2012f:20008)

[47] F. A. Smirnov, Form factors in completely integrable models of quantum field theory, Advanced Series in Mathematical Physics, vol. 14, World Scientific Publishing Co., Inc., River Edge, NJ, 1992. MR.1253319 (95a:81254) 
[48] L. A. Takhtadzhyan, Solutions of the triangle equations with $Z_{n} \times Z_{n}$-symmetry and matrix analogues of the Weierstrass zeta and sigma functions (Russian, with English summary), Zap. Nauchn. Sem. Leningrad. Otdel. Mat. Inst. Steklov. (LOMI) 133 (1984), 258-276. Differential geometry, Lie groups and mechanics, VI. MR742162 (85j:58064)

[49] O. Tsymbaliuk, The Affine Yangian of $\mathfrak{g l}_{1}$ and the Infinitesimal Cherednik Algebras, ProQuest LLC, Ann Arbor, MI, 2014. Thesis (Ph.D.), Massachusetts Institute of Technology. MR3279034

[50] M. van der Put and M. F. Singer, Galois theory of difference equations, Lecture Notes in Mathematics, vol. 1666, Springer-Verlag, Berlin, 1997. MR1480919 (2000e:39008)

[51] M. Varagnolo, Quiver varieties and Yangians, Lett. Math. Phys. 53 (2000), no. 4, 273-283, DOI 10.1023/A:1007674020905. MR.1818101(2002e:17023)

[52] E. T. Whittaker and G. N. Watson, A course of modern analysis, Cambridge Mathematical Library, Cambridge University Press, Cambridge, 1996. An introduction to the general theory of infinite processes and of analytic functions; with an account of the principal transcendental functions; Reprint of the fourth (1927) edition. MR.1424469 (97k:01072)

Department of Mathematics, Columbia University, 2990 Broadway, New York, New YORK 10027

E-mail address: sachin@math.columbia.edu

Department of Mathematics, Northeastern University, 567 Lake Hall, 360 Huntington Avenue, Boston, Massachusetts 02115

E-mail address: V.ToledanoLaredo@neu.edu 